San Jose State University

SJSU ScholarWorks

Master's Theses

Master's Theses and Graduate Research

Summer 2010

\title{
Electroless Deposition of Plasmonic Nanostructures on Star Polymer Templates
}

Melia Tjio

San Jose State University

Follow this and additional works at: https://scholarworks.sjsu.edu/etd_theses

\section{Recommended Citation}

Tjio, Melia, "Electroless Deposition of Plasmonic Nanostructures on Star Polymer Templates" (2010). Master's Theses. 3833.

DOI: https://doi.org/10.31979/etd.9uv6-kjpu

https://scholarworks.sjsu.edu/etd_theses/3833

This Thesis is brought to you for free and open access by the Master's Theses and Graduate Research at SJSU ScholarWorks. It has been accepted for inclusion in Master's Theses by an authorized administrator of SJSU ScholarWorks. For more information, please contact scholarworks@sjsu.edu. 


\title{
ELECTROLESS DEPOSITION OF PLASMONIC NANOSTRUCTURES ON STAR POLYMER TEMPLATES
}

\author{
A Thesis \\ Presented to \\ The Faculty of the Department of Chemical and Materials Engineering \\ San José State University \\ In Partial Fulfillment \\ of the Requirements for the Degree \\ Master of Science \\ by \\ Melia Tjio
}

August 2010 
(C) 2010

Melia Tjio

ALL RIGHTS RESERVED 
The Designated Thesis Committee Approves the Thesis Titled

\title{
ELECTROLESS DEPOSITION OF PLASMONIC NANOSTRUCTURES ON STAR POLYMER TEMPLATES
}

\author{
by \\ Melia Tjio

\begin{abstract}
APPROVED FOR THE DEPARTMENT OF CHEMICAL AND
MATERIALS ENGINEERING
\end{abstract} \\ SAN JOSÉ STATE UNIVERSITY
}

August 2010

$\begin{array}{ll}\text { Dr. Melanie McNeil } & \text { Department of Chemical and Materials Engineering } \\ \text { Dr. Roger Terrill } & \text { Department of Chemistry } \\ \text { Dr. Robert D. Miller } & \text { IBM Almaden Research Center } \\ \text { Dr. Joseph Sly } & \text { IBM Almaden Research Center }\end{array}$ 


\title{
ABSTRACT \\ ELECTROLESS DEPOSITION OF PLASMONIC NANOSTRUCTURES ON STAR POLYMER TEMPLATES
}

\author{
by Melia Tjio
}

As an alternative technique, electroless deposition is gaining interest due to its simplicity (solution-based process, no complex instrumentation), economical aspect, and effectiveness to form certain desired film thicknesses and morphologies on a wide variety of substrates. This work describes the use of water-soluble, polyvalent star polymer templates to form spontaneous self-limiting monolayers on complimentary functionalized substrates that act as efficient adhesion layers for the electroless deposition of thin gold films. The resulting gold films display outstanding thickness control, uniformity, reproducibility, and plasmon resonance generation, as evaluated by Rutherford backscattering spectroscopy (RBS), surface plasmon resonance (SPR) spectroscopy, and atomic force microscopy (AFM). The adaptation of this technique to the formation of core-shell gold nanoparticles was also shown to be feasible. The synthesized star polymer-templated gold nanoshells display a tunable NIR absorption depending on their size. By utilizing star polymers as templates, other materials such as drugs or chromophores can be effortlessly embedded inside the star polymer via self-assembled occlusion complex formation. This ability coupled with the efficacy of gold nanoshells in absorbing NIR light and transferring the light energy as heat to their surrounding environment can lead to an integrated form of imaging contrast, drug-delivery system, and photothermal ablation agent. 


\section{ACKNOWLEDGEMENTS}

I would like to thank Dr. Melanie McNeil who has provided me an once-in-alifetime opportunity to do my thesis at IBM Almaden Research Center (ARC). I really appreciate her support, advice, and help during the time I was working on my thesis and master's program at SJSU. I would also like to thank Dr. Joseph Sly for being a wonderful advisor whom I would be forever in-debt for what he has done for me during my tenure at IBM ARC. This included but not limited to his tremendous help, support, and his awe-inspiring knowledge that he willingly shared with me. I would also like to acknowledge great help from Victor Lee who has been an amazing lab master.

My sincere gratitude goes to Dr. Robert Miller for all his great feedback and input so I could obtain the best possible results in my research. I would also like to thank Dr. Jane Frommer, Dr. Andrew Kellock, Dr. Kumar Virwani, and Leslie Krupp who have shared their knowledge, trained me with state-of-the-art instruments, and provided me with thorough characterization analysis. My deepest appreciation also goes to my colleague students at IBM for their help and support. Last but not least, I would like to thank Dr. Roger Terrill for his keenness to be part of my committee members, all IBM ARC staffs, and my fellow students at SJSU. 


\section{TABLE OF CONTENTS}

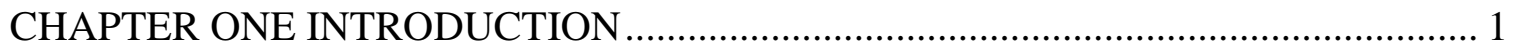

1.1 Gold Films via Electroless Deposition ......................................................2

1.2 Surface Plasmon Resonance (SPR) as Sensor Devices .................................4

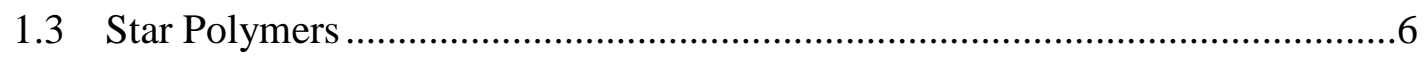

1.4 Gold Nanoshells ........................................................................................

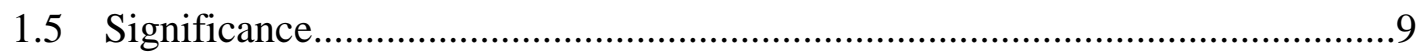

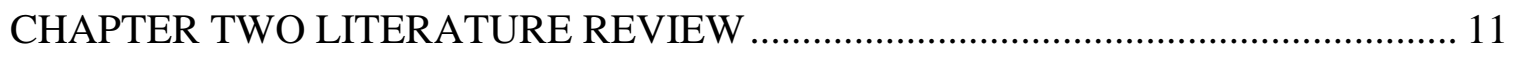

2.1 Hyperbranched Polymers as Building Blocks for Surface Modification.........11

2.2 Electroless Plating for 2D Thin Gold Film Formation ..................................13

2.2.1 Self-assembled Colloidal Gold as Seed Layer..................................13

2.2.2 Contiguous Gold Film from a Self-Assembled Colloidal Gold Seed Layer ...........................................................................19

2.3 Electroless Plating to Form 3D Gold Nanoshells ......................................25

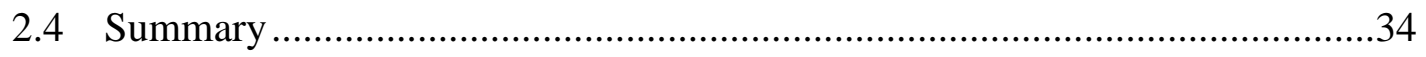

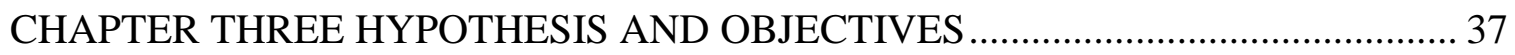

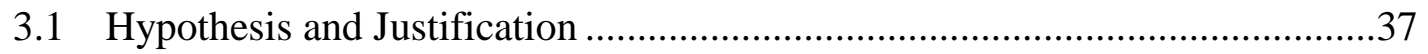

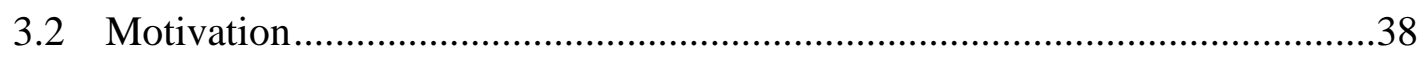

CHAPTER FOUR MATERIALS AND METHODS ................................................ 39

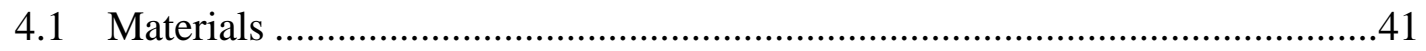

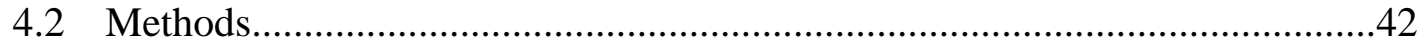

4.2.1 Quartz and SF 11 Surface Treatment ...........................................42

4.2.2 Star Polymer Solution Preparation for 2D Gold Films ......................43

4.2.3 Star Polymer Solution for 3D Gold Nanoshells ................................43

4.2.4 Citrate-capped Colloidal Gold Synthesis (for 2D Gold Films) ...........44

4.2.5 Gold/THPC Synthesis (for 3D Gold Nanoshells) ..............................44

4.2.6 Plated Gold Film Preparation.........................................................45 
4.2.7 3D Gold Nanoshell Synthesis .....................................................46

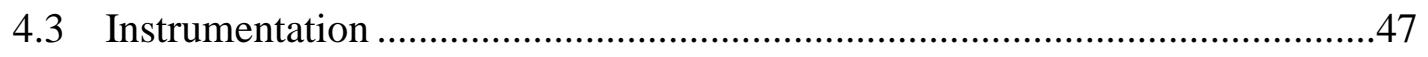

4.3.1 Circular Shaker ......................................................................47

4.3.2 UV-visible and Fluorescence Spectrophotometer .............................48

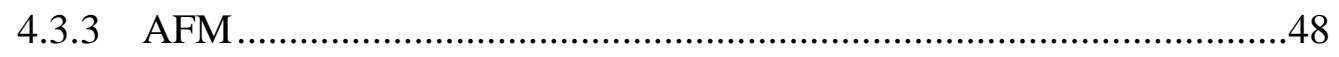

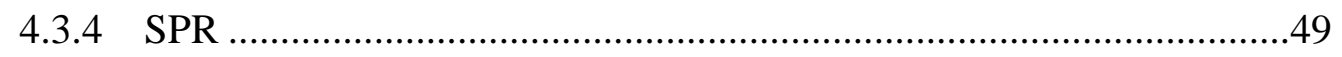

4.3.5 RBS, SEM, TEM, and EELS ....................................................50

4.3.6 Nanoindenter............................................................................50

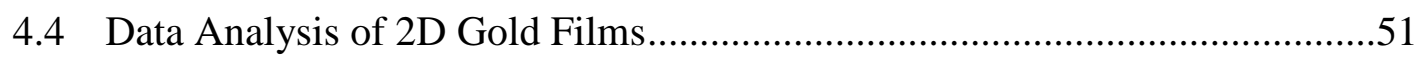

CHAPTER FIVE RESULTS AND DISCUSSION ................................................... 52

5.1 Self-assembled Star Polymer PS-DMAEMA as Adhesive Template..............53

5.2 Gold Colloid Immobilization as Seeding Step..............................................57

5.2.1 Gold Colloid Synthesis ...............................................................57

5.2.2 Gold Seed Attachment to Star Polymer Film .................................62

5.3 Growth/Plating Process to Form Contiguous Gold Films .............................65

5.3.1 Effects of Growth Solution Concentration ......................................65

5.3.2 Effects of Agitation on Film Uniformity ........................................74

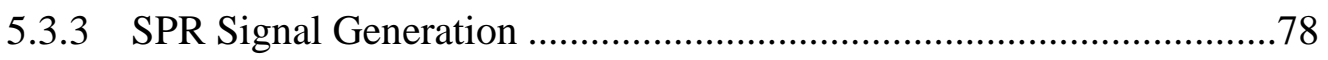

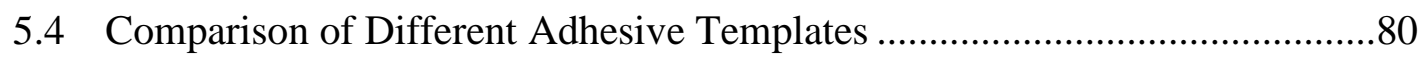

5.4.1 APTMS, PAH, and Star Polymer PS-DMAEMA Comparison............80

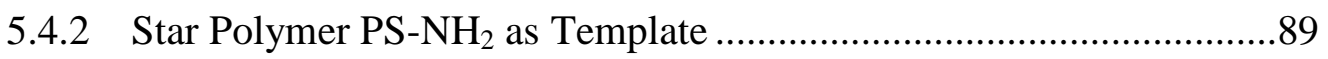

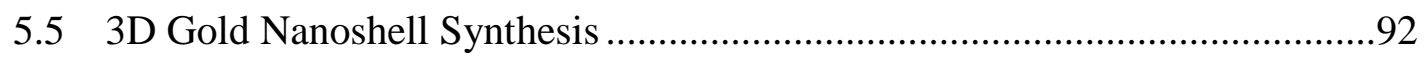

CHAPTER SIX CONCLUSIONS AND FUTURE WORK ..................................... 108

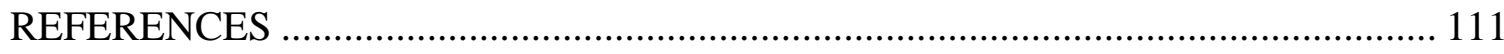

APPENDIX: An Example of Standard Deviation Calculation .................................. 118 


\section{LIST OF FIGURES}

Figure 1. Scheme of the gold seed enlargement by hydroxylamine without the formation of any new nucleation site [33]

Figure 2. Illustration of the generation of surface plasmon waves at a metal and dielectric medium interface [38].

Figure 3. A typical SPR setup based on the Kretchmann configuration. 5

Figure 4. Typical SPR substrates that are prepared by the vapor deposition method...

Figure 5. Structure of a star polymer with a polystyrene core and aminofunctionalized arms [44].

Figure 6. The efficacy of gold nanoshells in inducing photothermal ablation of tumor cells [45].

Figure 7. Compression of dendrimer multilayers in actual conditions [56].

Figure 8. UV-visible spectra of quartz slides that were immersed in an organosilane compound for 14 hours and colloidal gold for 24 hours [59]

Figure 9. AFM images $(500 \mathrm{~nm} \times 500 \mathrm{~nm}$ ) of a MPMDMS-coated glass and a $12 \mathrm{~nm} \mathrm{Au} / \mathrm{MPMDMS} /$ glass substrate [52].

Figure 10. AFM image $(1 \mu \mathrm{m} \times 1 \mu \mathrm{m})$ of a self-assembled layer of gold colloids on a mica substrate [64]

Figure 11. AFM image of self-assembled gold nanoparticles on a PEI/PSS/PAH/PSS/DMAP-coated quartz slide [66].

Figure 12. AFM images ( $1 \mu \mathrm{m}$ x $1 \mu \mathrm{m})$ of a $12 \mathrm{~nm}$ diameter colloidal $\mathrm{Au}$ monolayer on a MPTMS-coated glass before and after its immersion into a mixture of hydroxylamine hydrochloride and gold (III) chloride [33]

Figure 13. AFM images of a self-assembled gold colloid monolayer before and after the addition of hydroxylamine and gold (III) chloride [31].

Figure 14. SPR responses of electroless gold film with different plating times [41].

Figure 15. AFM images $(1 \mu \mathrm{m} \times 1 \mu \mathrm{m})$ of a plated gold film before and after annealing [25]. 
Figure 16. Plating time effects on the SPR responses of gold films [24].

Figure 17. UV-visible spectra depicting the progress of gold nanoshell

formation on $120 \mathrm{~nm}$ silica particles [46].

Figure 18. TEM images illustrating the growth process of gold nanoshell formation on $120 \mathrm{~nm}$ silica nanoparticles [46].

Figure 19. TEM images of the different distributions of gold colloids on silica particles that were functionalized with AEAPTMS, MPTMS, DPPETES, and non-functionalized [67]. .28

Figure 20. Illustration of gold nanoshell formation on a silica nanoparticle [68].

Figure 21. TEM images of colloidal gold attachment on the APTMS-

functionalized silica particles at different $\mathrm{pH}$ of gold colloid solution [69]

Figure 22. SEM images of silica nanoparticles that were coated with 23, 39, and $63 \mathrm{~nm}$ gold shell [18].

Figure 23. TEM images of silica particles with a $39 \mathrm{~nm}$ gold shell and a $82 \mathrm{~nm}$ gold shell that were obtained through the second growth step of the silica particles with $39 \mathrm{~nm}$ gold shell [18].

Figure 24. TEM images of gold nanoshell formation in a progressive order $[16]$

Figure 25. Schematic of magnetic gold nanocomposite synthesis [20]

Figure 26. Absorption at the NIR window ( $~ 850 \mathrm{~nm}$ in this case) was observed as after the formation of gold shell [20]

Figure 27. SPR instrument at IBM ARC.

Figure 28. Schematic illustration of 2D electroless gold film formation by utilizing star polymers as templates.

Figure 29. AFM images of a UV/ozone-treated silicon wafer before and after the deposition of star polymer PS-DMAEMA

Figure 30. AFM images of star polymer PS-DMAEMA (water base) that was deposited on a silicon wafer and a quartz wafer by dipping.

Figure 31. AFM images of star polymer PS-DMAEMA (water base) films that were formed in 5, 2, and 0.5 hour dipping time. 
Figure 32. The rapid formation of star polymer PS-DMAEMA (water base) layer as traced by SPR spectroscopy.

Figure 33. AFM images of star polymer PS-DMAEMA (water base) films that were produced from two different synthesis batches.

Figure 34. UV-visible spectra of a citrate-capped gold colloid solution.

Figure 35. TEM image of citrate-capped gold colloids with an absorption peak at $510 \mathrm{~nm}$ as shown in Figure 34.

Figure 36. Red-shifted UV-visible spectra of gold seed solution due to an increase in $\mathrm{NaBH}_{4}$ volume.

Figure 37. A narrower size distribution of the gold seeds was achieved by doubling the $\mathrm{NaBH}_{4}$ volume.

Figure 38. The repeatability of citrate-capped gold colloid synthesis as analyzed by UV-visible spectrophotometer.

Figure 39. The reproducibility of citrate-capped gold colloid synthesis as traced by UV-visible spectrophotometer....

Figure 40. SPR curves of citrate-capped gold colloid deposition on a star polymer-templated glass surface

Figure 41. UV-visible spectra of citrate-capped gold colloids before and after being immobilized on a star polymer-templated glass surface.

Figure 42. AFM images of a star polymer film on a glass surface before and after a seeding process ( $\mathrm{Z}$ height: $30 \mathrm{~nm}$ ).

Figure 43. The resulting different absorption levels due to a variation in seeding time.

Figure 44. Growth process of the seeded star polymer film as monitored in situ by SPR spectroscopy.

Figure 45. AFM images of gold seeded star polymer before and after the growth step with a $0.1 \%$ growth solution.

Figure 46. AFM image of a star polymer-templated gold film with a $20 \mathrm{~min}$ plating time and the corresponding SPR response.

Figure 47. Comparison of SPR responses from an electroless plated gold film and an evaporated gold film. 
Figure 48. Seeding and growth steps in the electroless gold film formation as characterized by UV-visible spectrophotometer.

Figure 49. Absorption spectra of a gold-seeded layer and plated gold films with various plating times $[26] .$.

Figure 50. Qualitative measurement of gold film thickness carried out using UV-visible spectrophotometer.

Figure 51. AFM images of star polymer-templated gold film that was plated for $1,2,3.5$, and $4.5 \mathrm{~min}$

Figure 52. Film thickness reproducibility of the star polymer-templated gold films.

Figure 53. Film thickness as a linear function of growth solution concentration (at a constant plating time).

Figure 54. The effects of agitation on gold film thickness.

Figure 55. Thickness variances were observed along one-inch wafers with the center area having $25 \%$ less thickness compared to the edges.

Figure 56. Floating technique resulted in films with consistently $25 \%$ less thickness at the center while the settling technique produced variable film thicknesses

Figure 57. Thicker films at edges despite having different agitation methods as shown by Okinaka et al. [74].

Figure 58. SPR curves of electrolessly deposited gold versus thermally deposited gold.

Figure 59. SPR curves generated from various batches of electroless gold films versus evaporated gold film.

Figure 60. Morphology of different adhesive templates as characterized by

$\operatorname{AFM}(1 \mu \mathrm{m} \times 1 \mu \mathrm{m})$

Figure 61. Various absorbance levels are shown by different templates (APTMS, PAH, and star polymer) at one hour seeding time.

Figure 62. AFM images of gold seed deposited on different adhesive templates (APTMS, PAH, and star polymer PS-DMAEMA). 
Figure 63. Gold film as grown on APTMS, star polymer, and PAH in 3 min plating time.

Figure 64. Lateral force measurement as a method to compare mechanical stability of star polymer-templated gold film to that of evaporated gold film and APTMS-templated or PAH-templated gold film.

Figure 65. Gold film as grown on APTMS, star polymer, and PAH under the conditions applied to star polymer.

Figure 66. PS-NH $\mathrm{NH}_{2}$ layer (DCM as the dissolving solvent) before and after an exposure to a gold seed solution and a growth solution.

Figure 67. PS- $\mathrm{NH}_{2}$ layer (toluene as the dissolving solvent) before and after an exposure to a gold seed solution and a growth solution.

Figure 68. Illustration of gold nanoshell formation utilizing star polymer as a template.

Figure 69. UV-visible absorption spectrum of gold/THPC.

Figure 70. DLS data of the gold/THPC that was used as seed in gold nanoshell formation.

Figure 71. A tunable NIR absorption from different sizes of star polymertemplated gold nanoshells

Figure 72. SEM images of different sizes of star polymer-templated gold nanoshells

Figure 73. SEM images of a crude sample of star polymer-templated gold nanoshells with observable excess salts and the crude sample after filtration with a $0.45 \mu \mathrm{m}$ glass filter

Figure 74. SEM images of a crude sample of star polymer-templated gold nanoshells before and after 24 hours dialysis against Millipore water and methanol.

Figure 75. SEM images of a crude sample of star polymer-templated gold nanoshells before and after a one-step centrifugation at 4,000 rpm for one hour.

Figure 76. SEM images of star polymer-templated gold nanoshells resulting from three different addition rates of hydroxylamine hydrochloride.

Figure 77. SEM image of $100 \mathrm{~nm}$ gold nanoshells from the fast addition of hydroxylamine hydrochloride in high magnification. 
Figure 78. Cross-section SEM image of star polymer-templated gold nanoshells

Figure 79. Bright-field and dark-field TEM images of star polymer-templated gold nanoparticles.

Figure 80. EELS spectra of star polymer-templated gold nanoparticles.

Figure 81. Illustration of possible interparticle interactions between seedsdecorated star polymers.

Figure 82. UV-visible spectra of star polymer/porphyrins-templated gold nanoshells before and after two days of THF dialysis.

Figure 83. At the same level of porphyrin concentration, there was a two-fold enhancement in the emission spectra of the star polymer-templated gold nanoshells compared to that of the 'bare' star polymer/porphyrin 


\section{LIST OF TABLES}

Table 1. The design of main experiments to form electroless $2 \mathrm{D}$ gold films.

Table 2. The design of experiments to form 3D gold nanoshells with star

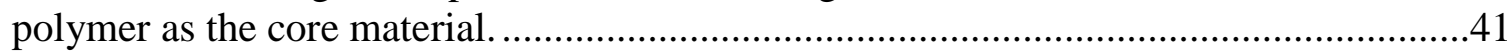

Table 3. Increasing gold film thicknesses resulting from longer plating times..............69

Table 4. Gold film thicknesses of seven samples from two batches, measured at five points across a $3 \mathrm{~mm}$ radial area.

Table 5. Conditions applied throughout the whole process for APTMS, PAH,

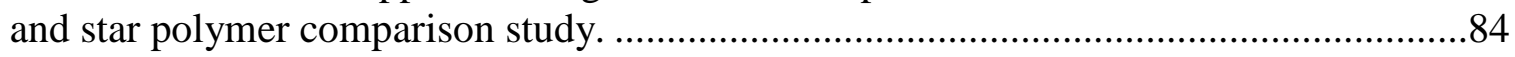

Table 6. Gold film thicknesses resulting from three different templates.......................85

Table 7. Gold film thicknesses resulting from three different templates under the conditions applied to star polymer. 


\section{CHAPTER ONE}

\section{INTRODUCTION}

Gold has been considered a precious metal since ancient times. For example, artisans have been using gold nanoparticles in the form of colloidal gold as a coloring substance for ceramic and decorative glasses for centuries. Colloidal gold has also been used for curative medicine or diagnostic tool for diseases such as epilepsy, heart problems, arthritis, and syphilis $[1,2]$. Besides the applications mentioned above, gold nanoparticles and thin films exhibit different properties than in their bulk configurations, the former of which are extremely useful for catalysis, in optics, for sensors, in biological, and microelectronic applications [3-8]. These properties include electronic, chemical, supramolecular, recognition, and physical properties such as surface plasmon resonance (SPR) behavior [1, 9].

The SPR behavior of gold nanoparticles in the form of thin films has been intensely exploited as the governing principle behind SPR spectroscopy. Thin gold films are usually obtained via vapor deposition (vacuum deposition) or by a sputtering technique. Since vapor deposition and sputtering require specific and expensive instruments, numerous studies have been conducted to develop an alternative technique called electroless deposition. This technique is useful as a means to overcome the drawbacks of existing methods because of its simplicity (solution-based process, no specific instrument such as vacuum chamber required), economical aspect, and effectiveness to form a desired film thickness and morphology on any surface, including surfaces that are electronically non-conductive [10-15]. 
The SPR behavior of a 3D thin gold film in the form of a nanoshell has drawn considerable interest as well. A dielectric core with a thin gold film acts as its shell to generate a tunable SPR property simply by changing the core-and-shell ratio [16]. The SPR property of gold nanoshells can be extended from the visible region to the infrared (IR) region, an optical property that is unattainable with solid gold nanoparticles [17-19]. Infrared light absorption is preferred for in situ biological studies since human tissues absorb minimally in the IR [17]. Potential applications for infrared light absorption by gold nanoshells include the use of gold nanoshells for contrast imaging and photothermal ablation agents in cancer treatment $[16,17,19,20]$.

\subsection{Gold Films via Electroless Deposition}

Electroless metal deposition, also called electroless plating, has been applied in the manufacturing of electronic devices for decades. This method has involved various metals such as nickel, palladium, and gold [21]. Electroless plating refers to the process of metal deposition through wet chemical reaction without the application of any electrical current. The process has been extended to any type of metal film formation from aqueous solution [22]. Electroless plating is an autocatalytic reduction and oxidation (redox) process using metal salts to deposit the corresponding metal onto a specified surface. The electron transfer between the reducing agent and metal salts (ions) is such that the reduction process occurs only on the catalyst-coated area. Otherwise the reduction process would happen in solution and not on the substrate surface $[15,23]$.

The electroless deposition method has been applied in the gold film formation for SPR substrates [24]. The whole process generally involves three main sequential steps: 
adhesive layer deposition on a glass substrate, self-assembly of gold colloids as a seed layer, and electroless plating to form the thin gold film. The adhesive layer is usually constructed from organosilane compounds with different functionality, such as amino, cyano, or mercapto groups. Linear polymers, such as poly(diallyldimethylammonium) chloride (PDDA), were also shown to be effective as adhesive materials in electroless gold deposition, as shown by Hrapovic et al. [25].

After an adhesive layer is formed on a glass substrate, the glass substrate is exposed to a gold nanoparticle solution. The gold nanoparticles will then form a selfassembled monolayer that covers the adhesive layer. The self-assembly process is achieved through electrostatic interaction between the gold colloids and the functional groups of the adhesive layer [26]. The gold nanoparticle layer acts as the catalyst and nucleation site for the formation of enlarged gold particles. The common methods to synthesize gold colloids are the citrate reduction method, the Brust-Schiffrin method, and the seeding growth method [1]. Gold colloids are readily available in the market in various sizes and with various stabilizers. The plating or growth process is initialized when the seeded substrate is introduced to a growth or plating solution that consists of gold ions and a reducing agent [24-28]. There are various reducing agents known to be effective for electroless gold film deposition including hypophosphite, hydrazine, dimethylamine borane (DMAB), and hydroxylamine [29-34]. These reducing agents are able to enlarge the seed particles without creating new nucleation sites, as shown in Figure 1. 


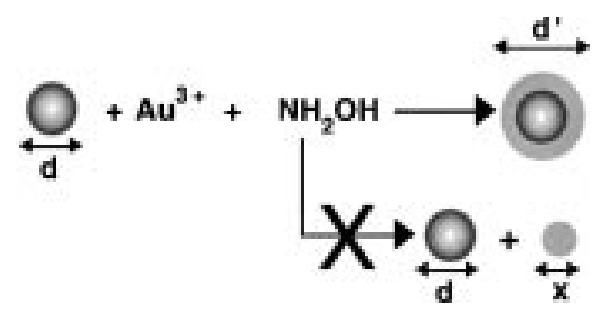

Figure 1. Scheme of the gold seed enlargement by hydroxylamine without the formation of any new nucleation site [33].

\subsection{Surface Plasmon Resonance (SPR) as Sensor Devices}

When light passes through two different media with different refractive indices (for instance glass and air), some fractions are reflected and some are refracted. At a certain angle of incidence, the whole fraction of light is reflected. This condition is called total internal reflection (TIR). Under TIR, monochromatic and plane-polarized light excites free electrons on a metal layer and causes them to move. The movement of the free electrons generates an evanescence field that penetrates through the metal layer and induces surface plasmon waves to occur at the interface between the metal and dielectric medium, as shown in Figure 2.

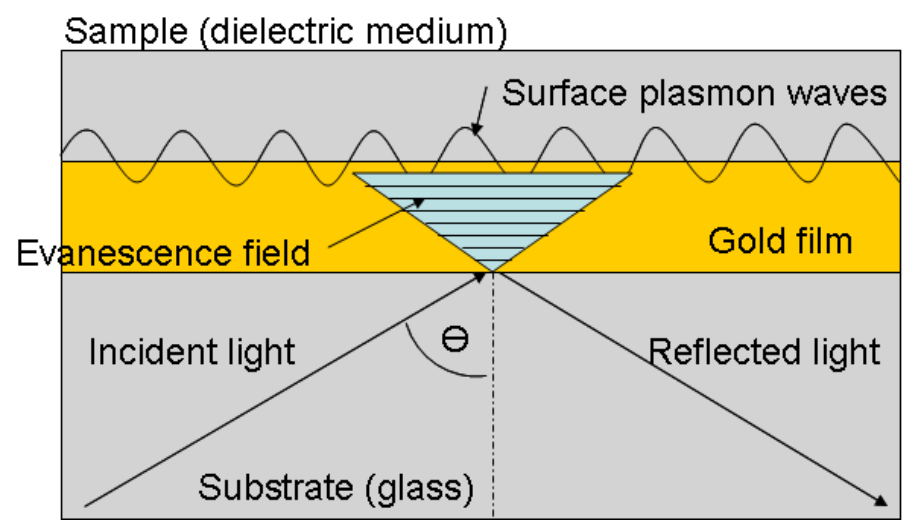

Figure 2. Illustration of the generation of surface plasmon waves at a metal and dielectric medium interface. $\Theta$ indicates the critical angle required to achieve a total internal reflection (TIR) condition [38] (reprinted with permission from Elsevier). 
The intensity of reflected light is less than incident light due to the energy transfer from the incident light to plasmon waves. At a certain incident light wavelength and angle, a minimum intensity in the reflected light can be observed. The angle where the minimum intensity of reflected light occurs is called the resonance angle or the surface plasmon resonance (SPR) angle. The SPR angle depends heavily on the refractive indices at both sides of the metal film. Thus, changes in the refractive index (such as when something binds to the exposed gold surface) will cause a change of SPR angle [35-37].

There are three main components of an SPR instrument: the optical system, the flow cell system, and the sensor surface (SPR substrate). Common configurations for the optical system include prism coupler-based, grating coupler-based, and optical waveguide-based systems. Most SPR instruments utilize the prism coupler-based configuration, more commonly known as the Kretchmann configuration [37, 39, 40]. A typical SPR setup based on the Kretchmann configuration is illustrated in Figure 3.

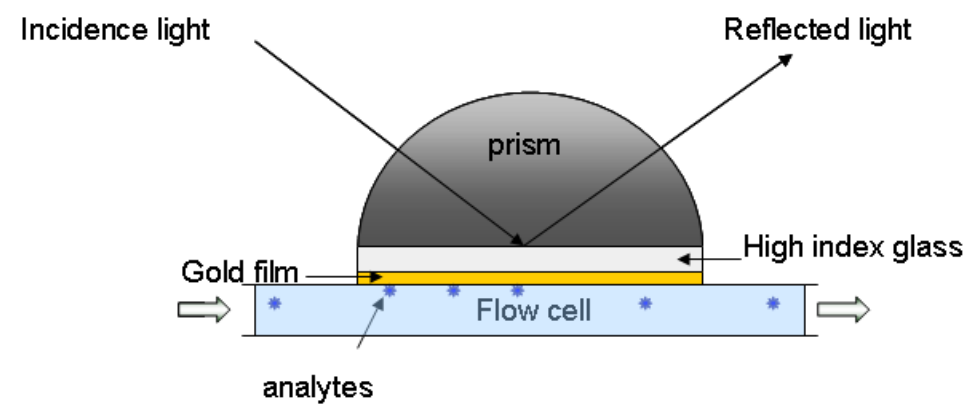

Figure 3. A typical SPR setup based on the Kretchmann configuration.

The SPR substrate usually consists of thin gold film $(45-60 \mathrm{~nm})$ deposited on a glass substrate [41]. Although other metals such as silver, copper, and aluminum could 
be used to coat the SPR substrate, gold film is most commonly chosen due to its stability and free electron behavior [38]. SPR substrates that are prepared by vapor deposition usually consist of thin chromium film as the adhesive layer and $50 \mathrm{~nm}$ gold film as shown in Figure 4.

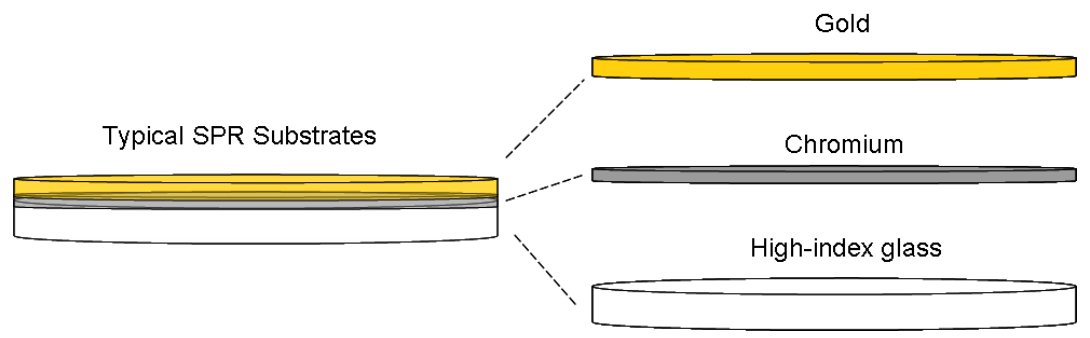

Figure 4. Typical SPR substrates that are prepared by the vapor deposition method.

\subsection{Star Polymers}

As part of this work, a star polymer would be explored as the building block for the adhesive layer in the electroless deposition of thin gold films. Although star polymers share a similar primary topography with dendrimers (core, branch unit with terminated functional groups, and interstitial region), star polymers have not been as well studied as dendrimers due to practicality issues and systemic problems in their synthesis $[42,43]$. Recent advancements in star polymer synthesis have brought great attention to this class of polymer structure. Sly et al. [43] showed that star polymers with complex structure and functionality could be easily synthesized to form uniform functional nanoparticles. Tunable molecular size is easily achieved by varying the number and size of the branch units. Chemical functionality of a star polymer is also modified by simply changing the functional groups at periphery [43]. Figure 5 shows the structure of a star polymer with a polystyrene core and amino functional groups at the peripheral ends. 
This particular structure was the one used in this research project. The star polymer structure also allows the incorporation of other materials inside the interstitial space. Sly et al. [43] showed that they were able to embed porphyrins and organic dyes inside the star polymer via a self-assembled occlusion complex formation.

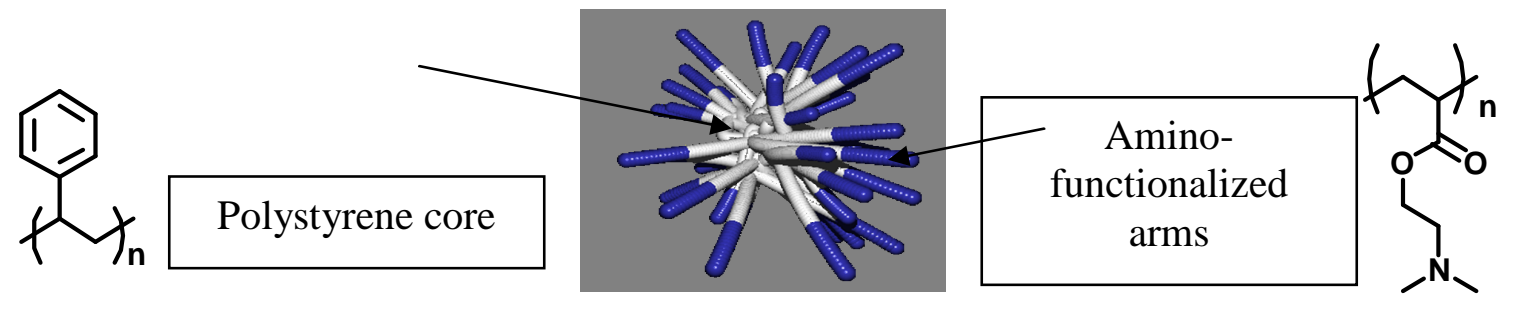

Figure 5. Structure of a star polymer with a polystyrene core and amino-functionalized arms [44] (reprinted with permission from Joseph Sly).

\subsection{Gold Nanoshells}

The ability to use star polymers as templates for the electroless deposition of gold films would also open up the potential for their use as templates for the formation of 3D gold nanoshell particles. Solid gold nanoparticles are used in numerous applications due to their unique optical and electronic properties. One of these unique properties is the strong surface plasmon resonance (SPR) absorption in the visible region [16]. For example, $5 \mathrm{~nm}$ spherical solid gold particles in an aqueous media exhibit a strong absorption at $520 \mathrm{~nm}$. An increase in particle size, however, does not effectively shift the absorption range of the spherical solid gold particles. For instance, an increase in particle size from $5 \mathrm{~nm}$ to $80 \mathrm{~nm}$ only shifts the absorption peak by $25 \mathrm{~nm}$ [18].

On the other hand, the absorption of gold nanoshells can be broadly tuned from the visible range to the near infrared (NIR) range. This absorption in the NIR range is the major driving force that attracts intensive studies in gold nanoshells. Irradiation with 
NIR light onto human tissues generates a minimal amount of thermal injuries to normal tissues [18]. Therefore, the use of nanoshells that absorb NIR light is ideal for in situ biological studies. Upon absorbing NIR light, gold nanoshells transfer the light energy into thermal energy to surrounding tissues [17]. The thermal energy (heat) then induces the localized destruction of cells such as tumor cells. Figure 6 shows the efficacy of gold nanoshells in inducing photothermal ablation of tumor cells upon laser irradiation [45]. In this particular study, gold nanoshells were injected into solid tumors in female mice with severe combined immunodeficiency (SCID). Irradiation with NIR light (820 nm) for less than 6 minutes resulted in cell death, as shown in Figure 6C).

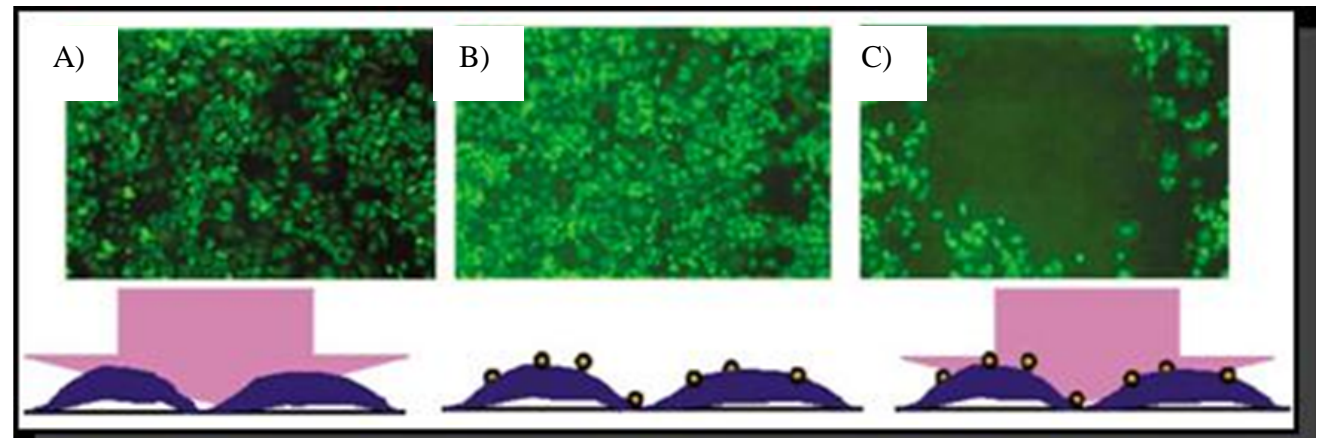

Figure 6. The efficacy of gold nanoshells in inducing photothermal ablation of tumor cells. A) control cells without gold nanoshells, B) cells with gold nanoshells prior to radiation, and $\mathrm{C}$ ) cells with gold nanoshells post-radiation (the dark region indicates cell death) [45].

The synthesis of gold nanoshells was first reported by Oldenburg et al. [46] in 1999. Since then, their procedure to synthesize gold nanoshells has been adapted by numerous researchers. The procedure generally includes four steps. The first step is the synthesis of silica nanoparticles for core materials. The second is the functionalization of the silica nanoparticles with amine or thiol linker groups. Following the functionalization 
step is the attachment of small gold particles (gold seeds) to the amine/thiol groups on the silica surface. The final step is the enlargement of the gold seeds by adding an appropriate growth solution. The enlarged gold particles will coalesce and form a continuous gold layer or shell on the outer surface of the silica particles.

Silica nanoparticles are chosen as the core material in most studies due to their low size polydispersity and simplicity in their synthesis via the Stöber method. However, complex additional steps are required to incorporate additional materials such as fluorescent dyes into silica nanoparticles. On the other hand, star polymers offer an instantaneous formation of occlusion complex with the fluorescent dyes by utilizing solvent solubility differences of star polymers and dyes. The amine groups which are readily available on the periphery of star polymers also eliminate the need for the additional functionalization steps in forming the nanoshell templates. Consequently, as a separate of this work, star polymers would be explored as templates for the formation of 3D gold nanoshell particles.

\subsection{Significance}

SPR spectroscopy has been widely demonstrated as a powerful sensing instrument. It allows real-time and tagless detection of various biological or chemical molecules in biotechnology, biomedical, and environmental studies [38, 47-50]. The electroless plating method used to construct the gold films for SPR substrates allows wet chemical laboratories to perform this process without any instrument or financial limitations such as the need for vacuum chambers [10-14]. This electroless method also 
allows selective gold film deposition on a patterned surface, potentially of value to the microelectronic device industry.

Furthermore, the electroless process can be utilized as a platform to generate gold nanoshells with a star polymer as the core material which enables specific dyes or hydrophobic drugs to be incorporated inside the core. The use of embedded dyes can potentially enhance the ability of gold nanoshells in absorbing infrared light and in scattering light which makes them more ideal for photoimaging agents and photothermal ablation agents in cancer treatment. Irradiation with certain laser energy is shown to induce destruction of gold nanoshells [51]. If drug-embedded star polymers are used as the core materials, the destruction of gold nanoshells will lead to the drug release. This ability coupled with the efficacy of gold nanoshells in absorbing NIR light and transferring the light energy as heat to surrounding cells provides the potential for an integrated form of imaging contrast, drug-delivery system, and photothermal ablation agent. 


\section{CHAPTER TWO}

\section{LITERATURE REVIEW}

One prerequisite for electroless plating to take place is the availability of a seed layer of catalytic sites. Colloidal gold is commonly used for seeds in electroless gold plating for several reasons. First, colloidal gold can be easily prepared in an aqueous solution, and it is stable for a defined period of time. Second, colloidal gold can be obtained in a broad range of sizes $(2.5-120 \mathrm{~nm})$ with a fairly narrow size distribution [52]. Colloidal gold is generally immobilized on an activated or functionalized surface through a self-assembly process. Surface functionalization itself is usually achieved by the deposition of organosilane compounds. The plating process is initiated when a suitable mixture of gold salt and reducing agent is added to a colloidal gold-coated substrate. In this chapter, prior studies pertinent to surface functionalization, selfassembled colloidal gold monolayer formation, and electroless gold plating process in the formation of 2D and 3D gold nanostructures are reviewed.

\subsection{Hyperbranched Polymers as Building Blocks for Surface Modification}

Since the first synthesis of dendrimers was reported over twenty years ago, numerous studies have been carried out to explore the applications of dendrimers in chemical, biological, and medicinal fields. Dendrimers are distinctively known for their regular structure and chemical versatility [42]. Although star polymers share the same topographical features as dendrimers (branch units originating from a core, terminal 
functional groups, and interstitial region), there are fewer studies addressed to the investigation of star polymers owing to the traditionally more difficult synthesis $[42,43]$.

The first study of confined-surface dendrimers deposition was performed by Watanabe and Regen [53]. In their study, they demonstrated that poly(amidoamine) dendrimers (PAMAM) generation 6 could form a multilayer construction. As a result, they suggested that multilayer dendrimers opened a new class of thin films that had unique and exploitable properties.

Wells and Crooks [54] later studied the formation of a monolayer of PAMAM dendrimers on surfaces. They used different generations of PAMAM in their research and discovered that higher generations of PAMAM (Generation 4) were more reactive and selective compared to non-spherical dendrimers (Generation 0 and 2). The formation of dendrimer bilayers on surfaces was also shown by Tokuhisa and Crooks [55].

A comprehensive study on the self-assembled multilayer of dendrimers via electrostatic interactions was performed by Tsukruk et al. [56]. One interesting phenomena observed from their experimental results was the compression of dendrimers in actual conditions compared to ideal conditions, as illustrated in Figure 7.
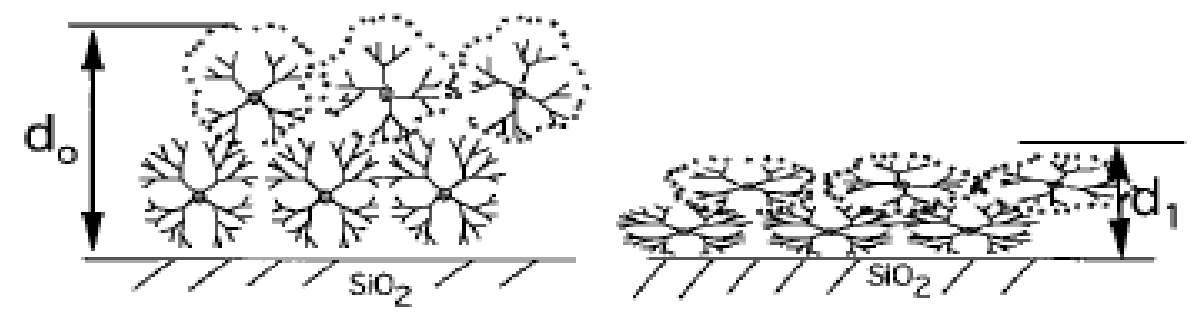

Figure 7. Compression of dendrimer multilayers in actual conditions [56] (reprinted with permission from American Chemical Society). 
The use of star polymers as thin film building blocks on surfaces was demonstrated by Bonifacio and Chang $[57,58]$. Bonifacio showed that the multilayer formation of star polymer via electrostatic interactions was feasible in minutes. Bonifacio and Chang $[57,58]$ utilized a real-time SPR detector as the main characterization unit to investigate the star polymer deposition. AFM was also performed in their study to verify the stability of the formed films.

\subsection{Electroless Plating for 2D Thin Gold Film Formation}

\subsubsection{Self-assembled Colloidal Gold as Seed Layer}

In a study conducted by Grabar et al. [59], different types of organosilane compounds were used to anchor colloidal gold on glass and quartz. The organosilanes were 3-aminopropyltrimethoxysilane (APTMS), 3-cyanopropyldimethylmethoxysilane (CPDMMS), 3-mercaptopropyl methyldimethoxysilane (MPMDMS), and 3cyanopropyltriethoxysilane (CPTES). The different functional groups of organosilane compound (cyano, amino, and thiol) are known to have a high affinity toward gold colloids. Thus, these functional groups bind with colloidal gold while the alkoxy groups of organosilane react with the hydroxyl groups that are naturally available on glass [59]. The gold colloid utilized in their study was synthesized through the reduction of gold chloride salts by sodium citrate. In their study, they showed that the colloidal gold immobilization on glass could be monitored by UV-visible spectrophotometer and transmission electron microscopy (TEM). A gold colloid with a $13 \mathrm{~nm}$ diameter in solution showed a maximum absorption peak $\left(\lambda_{\max }\right)$ at $520 \mathrm{~nm}$, while in immobilized form the maximum peak $\lambda_{\max }$ was shifted to the range of $600-800 \mathrm{~nm}$. Figure 8 shows the 
UV-visible spectra of quartz slides that were coated with organosilane compound and colloidal gold [59].

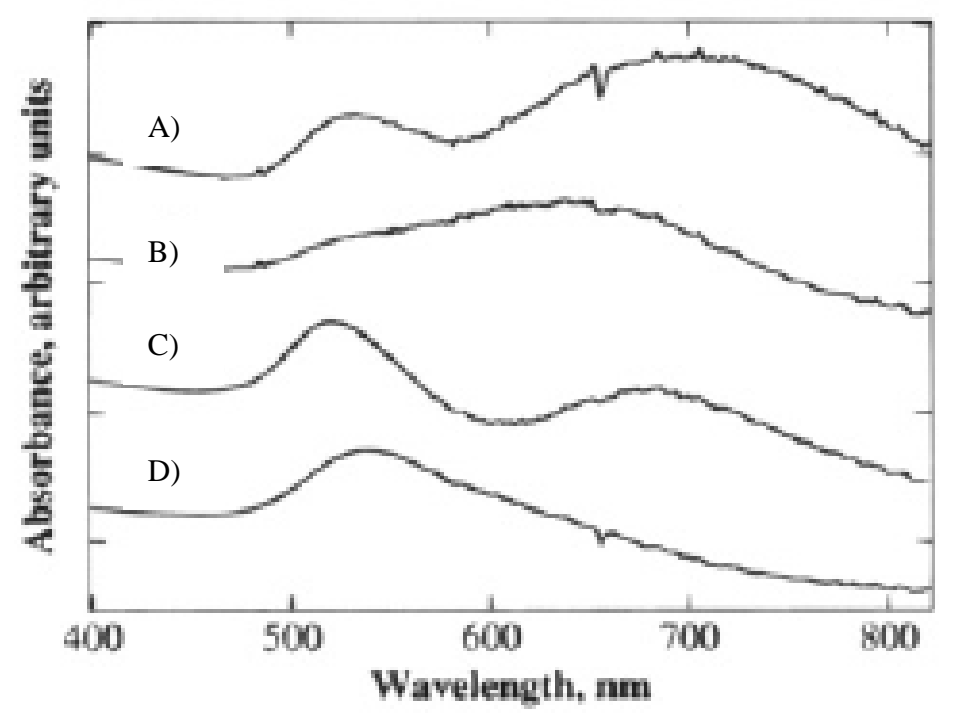

Figure 8. UV-visible spectra of quartz slides that were immersed in an organosilane compound for 14 hours and colloidal gold for 24 hours: A) APTMS, $30 \mathrm{~nm} \mathrm{Au}$; B) APTMS, $13 \mathrm{~nm} \mathrm{Au}$; C) MPMDMS, $30 \mathrm{~nm} \mathrm{Au}$; and D) MPMDMS, $13 \mathrm{~nm}$ Au [59] (reprinted with permission from American Chemical Society).

In a later publication, Grabar et al. [52] used smaller gold colloids to investigate colloidal gold size effect on the self-assembly interaction between gold nanoparticles and functional groups of organosilanes. The citrate-reduction method that was used in their previous study proved to be robust for 10-40 nm diameter gold colloid synthesis. Thus, in order to make smaller seeds (below $10 \mathrm{~nm}$ ) they utilized sodium borohydride $\left(\mathrm{NaBH}_{4}\right)$ instead of citrate as a reducing agent $\left(\mathrm{NaBH}_{4}\right.$ is a stronger reducing agent compared to citrate). It was concluded that colloidal gold size did not demonstrate a significant effect on the affinity towards functional groups of organosilanes. However, the colloidal gold obtained from $\mathrm{NaBH}_{4}$ reduction depicted a lower binding strength to aminosilanes and 
mercaptosilanes compared to the gold from citrate reduction [52]. In this study, Grabar et al. [52] employed atomic force microscopy (AFM) as an additional instrument in monitoring colloidal gold deposition. Figure 9 shows the AFM images of a glass surface covered with MPMDMS (left) and a glass/MPMDMS surface covered with $12 \mathrm{~nm}$ gold colloid (right).
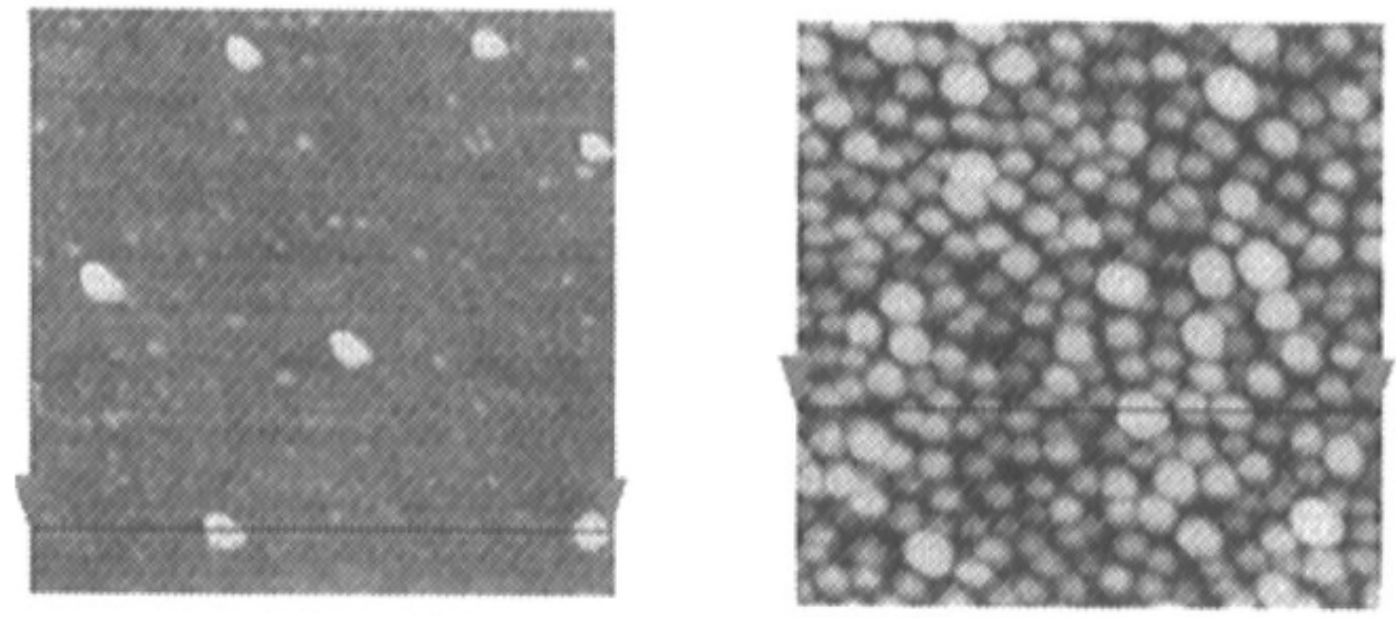

Figure 9. AFM images (500 nm x $500 \mathrm{~nm}$ ) of a MPMDMS-coated glass (left) and a 12 $\mathrm{nm} \mathrm{Au} / \mathrm{MPMDMS} / \mathrm{glass}$ substrate (right) [52] (reprinted with permission from American Chemical Society).

Colloidal gold self-assembly on an organosilanes-functionalized surface was also studied by Zheng et al. [60]. Instead of glass, they used silicon substrates that were surface-modified by exposing the substrates to a mixture of ammonium hydroxide and hydrogen peroxide [61]. This surface modification was intended to make the silicon surface rich with silanol groups. After this surface modification step, the substrates were immersed in an aminopropyltriethoxysilane (APTES) solution. Colloidal gold with 12 nm diameter was introduced to the APTES-coated substrates. The alkoxy groups of 
APTES formed covalent $\mathrm{Si}-\mathrm{O}$ bonds with the silanol groups on silicon substrates, while the amino groups of APTES bound the colloidal gold.

Liu et al. [62] later performed a similar study to the one conducted by Zheng et al. [60]. In their experiments, APTES-coated silicon substrates were soaked in a colloidal gold solution for 12 hours to enable electrostatic interaction between the amino groups of APTES and the citrate-capped colloidal gold. They concluded that only a sub-monolayer of colloidal gold was formed [62]. Thus, they soaked the sub-monolayer colloidal goldcoated silicon in a dodecane-1-thiol $\left(\mathrm{Me}\left(\mathrm{CH}_{2}\right)_{11} \mathrm{SH}\right)$ solution for 24 hours. Due to a higher affinity between sulfur and gold particles, the gold particles were capped with sulfur/thiol molecules instead of citrate molecules. Electrostatic interaction between the citrate-capped gold particles and amino groups was replaced by the Van der Waals attraction allowing the gold particles to move and aggregate. Alcohol solvent was added and allowed to evaporate in order to eliminate the interstitial spaces between domains of gold particles. The results indicated that evaporation led to a densely continuous monolayer of colloidal gold.

Organosilanes such as APTES and 3-mercaptopropyltrimethoxysilane (MPTMS) were also used in a study elaborated by Kang et al. [63]. The APTES-coated silicon wafers were immersed in a colloidal gold solution containing gold particles with an average size of $14 \mathrm{~nm}$. SEM imaging verified that gold colloids were attached to the amino groups of APTES [63]. Guo and coworkers [64] also demonstrated the effectiveness of organosilane compounds as anchoring molecules for colloidal gold monolayer formation. They immersed glass substrates in an APTES solution overnight 
and allowed the APTES-coated glasses to come into contact with a colloidal gold solution for a period of 3 hours. The gold colloids used in this study were obtained from the citrate-reduction method with an average diameter of $16 \mathrm{~nm}$ [64]. The gold colloid immobilization was characterized by UV-visible spectrophotometer and AFM. Figure 10 shows the AFM image of the self-assembled gold colloids on a mica substrate.

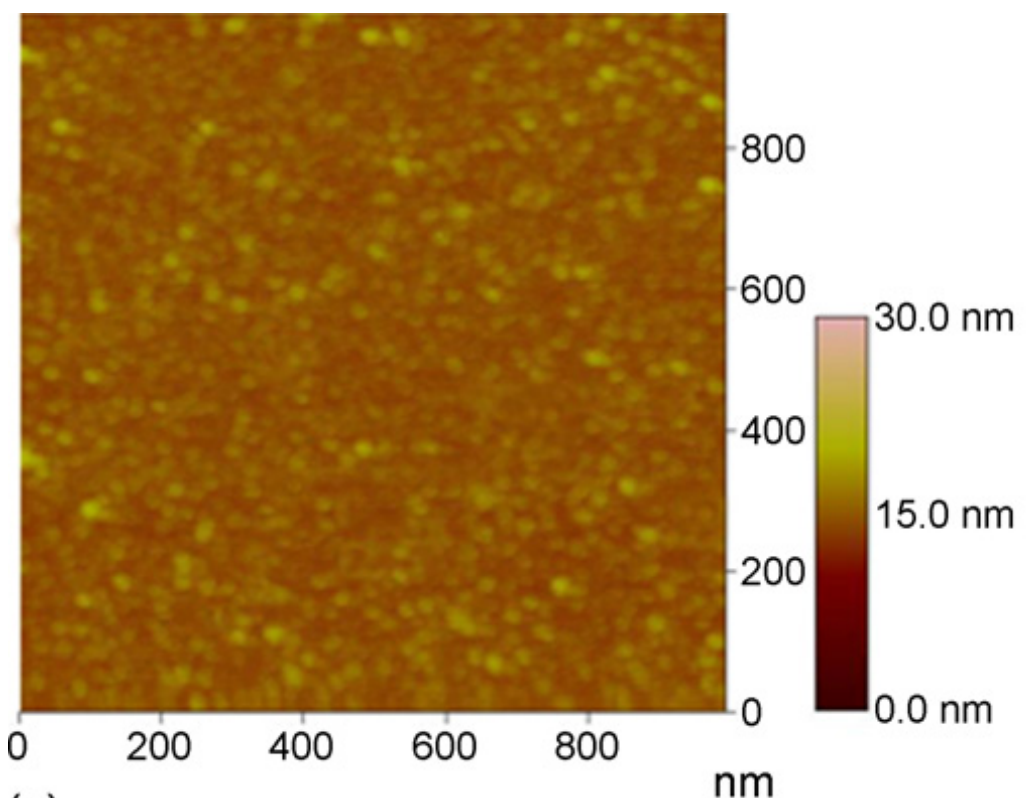

Figure 10. AFM image $(1 \mu \mathrm{m} \times 1 \mu \mathrm{m})$ of a self-assembled layer of gold colloids on a mica substrate [64] (reprinted with permission from Elsevier).

The work reported by Jung et al. [65] in 2007 further confirmed the use of organosilanes (MPTMS in particular) to anchor colloidal gold on silicon substrates. The colloidal gold used in their study was synthesized by the reduction of gold (III) chloride $\left(\mathrm{HAuCl}_{4}\right)$ by trisodium citrate and $\mathrm{NaBH}_{4}$. Their study showed that smaller gold particles $(5.5 \mathrm{~nm}$ and $13 \mathrm{~nm})$ formed a good closed-packed structure compared to larger gold particles $(30 \mathrm{~nm}$ ) [65]. When multiple layers of gold nanoparticles were built, the 
layers with an initial layer composed of smaller gold nanoparticles exhibited less surface roughness [65].

Besides organosilanes, diazo resins (DAR) were also found to be effective as gold colloid cross-linkers in a study conducted by Bai and coworkers [8]. In their study, sulfonated quartz wafers were sequentially soaked in DAR solution followed by colloidal gold to form the self-assembled DAR-gold nanoparticles sandwiches. UV irradiation was applied on these DAR-gold nanoparticles sandwiches to change the multilayers' electrostatic interaction into covalent bond [8]. UV-visible spectrophotometer and AFM were the main characterization instruments used to examine the formation of selfassembled gold nanoparticle layers.

Gittins and colleagues [66] successfully utilized polyelectrolytes as anchoring molecules for a self-assembled colloidal gold layer on surfaces. Four layers of polyelectrolytes $(\mathrm{PE})$ were applied to the surface of a quartz wafer before colloidal gold was introduced. The four different types of PE were poly(ethyleneimine) (PEI), poly(styrenesulfonate) (PSS), poly(allylamine hydrochloride) (PAH), and poly(styrene) sphere (PSS). According to Gittins et al. [66] PE layers provided a relatively uniform charge across the quartz wafer that was essential for uniform colloidal gold adsorption. The PE-modified substrates also showed a denser loading of gold particles compared to unmodified ones [66]. Gittins et al. [66] used 4-dimethylaminopyridine (DMAP), an organic base, as the colloidal gold stabilizer instead of citrate as used by other researchers. The self-assembled colloidal gold formation was observed using UV-visible spectrophotometer and AFM. 
Figure 11 presents the AFM image of colloidal gold deposited on a thin film of PEI/PSS/PAH/PSS. From this figure, it is apparent that a single deposition process resulted in a dense loading of gold particles. The surface roughness for this film was $7 \pm$ $2 \mathrm{~nm}$, suggesting some aggregation of gold particles [66].

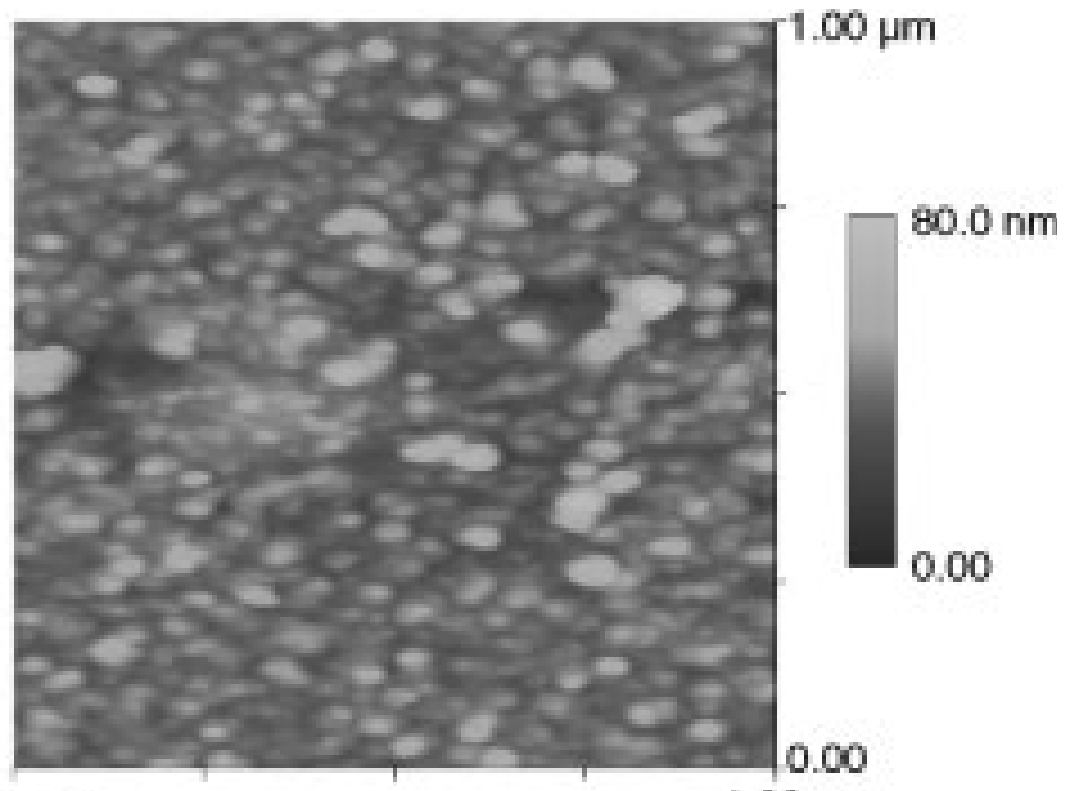

Figure 11. AFM image of self-assembled gold nanoparticles on a PEI/PSS/PAH/PSS/DMAP-coated quartz slide [66]. Copyright Wiley-VCH Verlag GmbH \& Co. Reproduced with permission.

\subsubsection{Contiguous Gold Film from a Self-Assembled Colloidal Gold Seed Layer}

Brown and Natan [33] were among the first researchers to demonstrate the ability of hydroxylamine hydrochloride to enlarge colloidal gold nanoparticles without creating any new nucleation sites. In their study, they used a seed layer of $12 \mathrm{~nm}$ diameter colloidal gold nanoparticles self-assembled on a MPTMS-coated glass. The gold nanoparticles were then enlarged by the addition of hydroxylamine hydrochloride and gold (III) chloride. AFM scanning showed that $12 \mathrm{~nm}$ diameter gold nanoparticles were 
enlarged up to $50 \mathrm{~nm}$ [33]. AFM images revealed that larger particles reduced the interstitial space between particles and formed a denser layer of gold. The AFM images of gold nanoparticles monolayer, before and after the addition of hydroxylamine hydrochloride and gold salts, are shown in Figure 12.
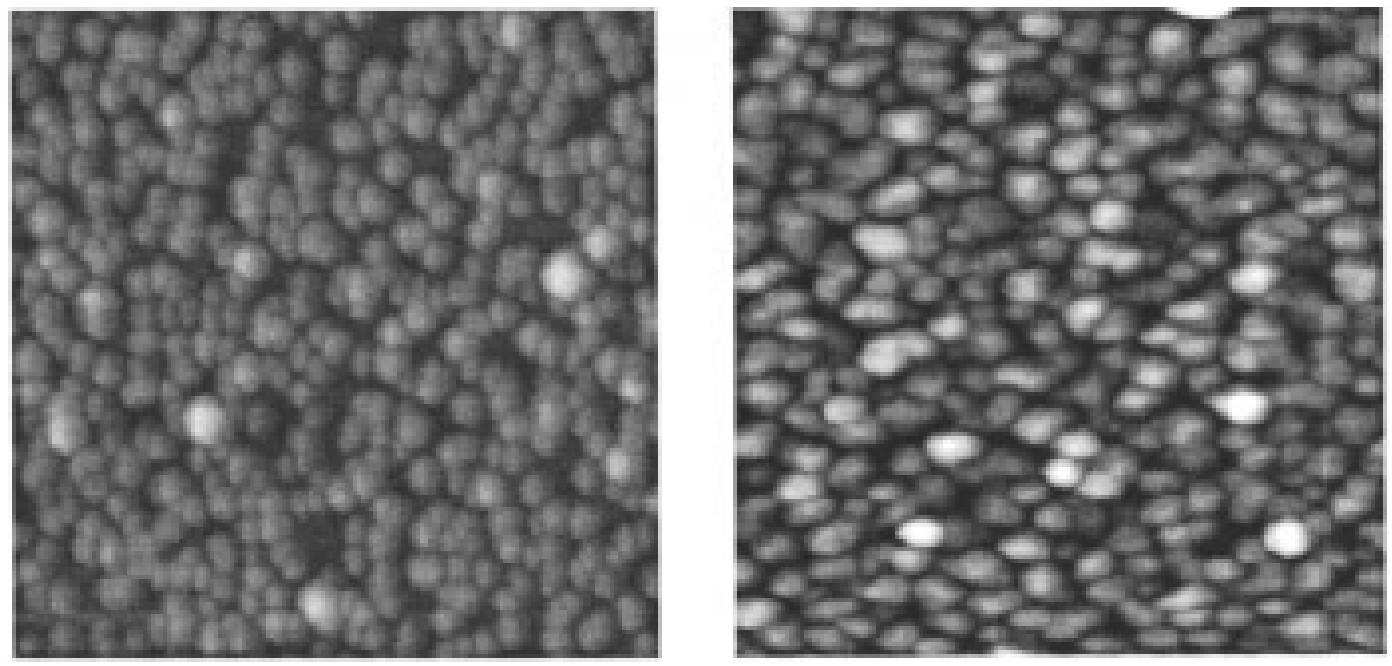

Figure 12. AFM images $(1 \mu \mathrm{m} \times 1 \mu \mathrm{m})$ of a $12 \mathrm{~nm}$ diameter colloidal Au monolayer on a MPTMS-coated glass: before (left) and after its immersion into a mixture of hydroxylamine hydrochloride and gold (III) chloride (right) [33] (reprinted with permission from American Chemical Society).

Menzel et al. [31] further confirmed the hydroxylamine hydrochloride capability to enlarge surface-confined gold nanoparticles. In their study, $20 \mathrm{~nm}$ diameter colloidal gold nanoparticles were self-assembled on a 3-mercaptopropyldimethoxymethylsilanecoated $\mathrm{SiO}_{2}$ surface. A mixture of hydroxylamine hydrochloride and gold (III) chloride was sequentially added onto the colloidal gold nanoparticles monolayer. The addition enlarged the gold particle and increased the plated film height by up to $75 \mathrm{~nm}$. The surface roughness of the plated gold film was also increased as verified by AFM. From their experimental results, Menzel et al. [31] concluded that particle distribution, solution 
concentration, and agitation were the three main factors that affected the plated film thickness and roughness [31]. The AFM images of colloidal gold monolayer, before and after plating, are shown in Figure 13.
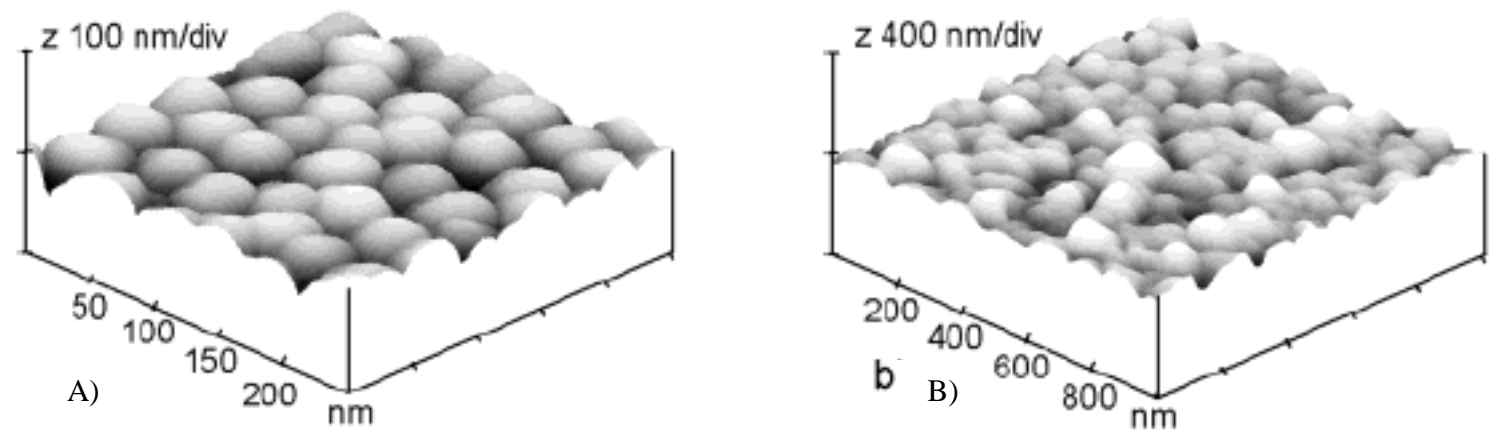

Figure 13. AFM images of a self-assembled gold colloid monolayer: A) before and B) after the addition of hydroxylamine and gold (III) chloride [31]. Copyright Wiley-VCH Verlag GmbH \& Co. Reproduced with permission.

A gold plating process on an immobilized colloidal gold by hydroxylamine hydrochloride was also investigated in the late 1990's by Musick and co-workers [34]. Glass substrates were immersed in an APTMS solution to provide chemisorption sites for colloidal gold nanoparticles. After a colloidal gold monolayer was formed, a mixture of hydroxylamine hydrochloride and gold salts was added as a plating solution. Enlarged gold nanoparticles were rapidly developed and a conductive gold film was formed as the plating time increased. The plated gold formation was monitored by UV-visible/NIR spectrophotometer and AFM [34].

Another study on the use of hydroxylamine hydrochloride as a reducing agent for gold nanoparticles growth was also carried out by Meltzer et al. [32]. In their study, they showed that hydroxylamine could be used to enlarge gold nanoparticles and form gold 
nanowires. Scanning probe microscopy (SPM) was used as the main characterization device in this study [32].

The specific application of electroless gold film deposition for SPR substrates was initially studied in 2001 by Jin et al. [41]. They coated a glass substrate with APTMS by simply immersing the glass in an APTMS solution for 12 hours. The APTMS-coated glass was then soaked in a colloidal gold solution for 18 hours. An aqueous solution of $0.4 \mathrm{mM}$ hydroxylamine and $0.1 \%$ gold (III) chloride was added to form a continuous gold film from the colloidal gold monolayer. According to their experiment, a definite color change on the glass substrates from pink to blue to finally gold luster was observed. They also showed that the gold film thickness could be adjusted by changing plating time. SPR responses from different plating time are shown in Figure 14.

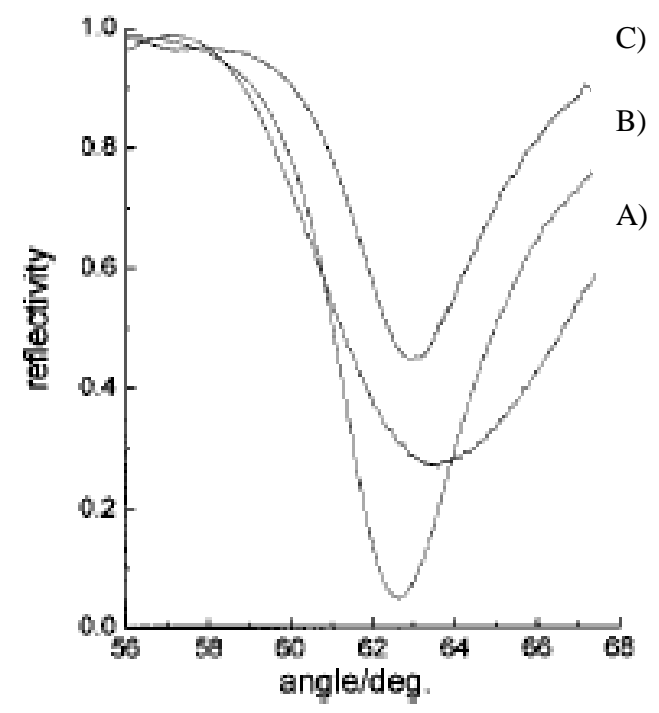

Figure 14. SPR responses of electroless gold film with different plating times: A) 5 min, B) $10 \mathrm{~min}$, and C) $15 \mathrm{~min}$ [41] (reprinted with permission from American Chemical Society). 
In 2003, Hrapovic et al. [25] reported that they were also able to prepare a continuous thin gold film on glass surfaces functionalized with poly(diallyldimethylammonium) chloride (PDDA) instead of the typical organosilane compounds. A self-assembled colloidal gold monolayer formed on this PDDA layer via electrostatic interactions after 18 hours of immersion. Two different reducing agents (formaldehyde and hydroxylamine hydrochloride) were compared in this study. Experimental results indicated that the hydroxylamine hydrochloride provided a faster route to enlarge gold nanoparticles. From their study, it was found that film roughness increased with film thickness. Thermal annealing at $250{ }^{\circ} \mathrm{C}$ for 2 hours was conducted to evaluate its effect on surface roughness and morphology. The shape of enlarged gold nanoparticles changed significantly after thermal annealing but the surface roughness remained approximately the same, as shown by the AFM images in Figure 15.
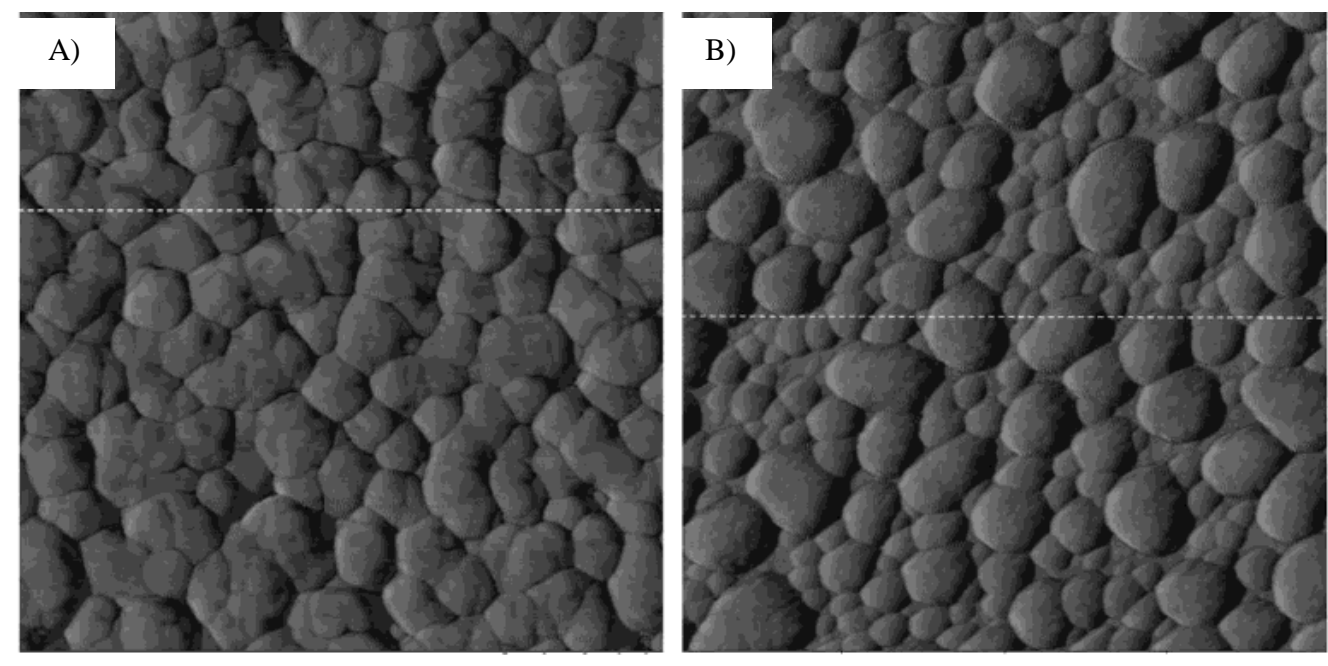

Figure 15. AFM images ( $1 \mu \mathrm{m} \times 1 \mu \mathrm{m})$ of a plated gold film: A) before annealing, roughness $\mathrm{Ra}=10.50 \mathrm{~nm}$ and $\mathrm{B}$ ) after annealing, roughness $\mathrm{Ra}=9.7 \mathrm{~nm}$ [25] (reprinted with permission from American Chemical Society). 
Huang and co-workers [26] proposed a different approach in preparing thin gold films on glass via electroless plating. They hypothesized that it was unnecessary to have an adhesive layer as suggested by other studies. A microwave oven was utilized to facilitate the self-assembly process of colloidal gold on a naked glass. Their experimental results showed that the self-assembled gold monolayer was formed after 6 minutes of microwave irradiation. Immersion of the seeded glass in $0.1 \%$ gold (III) chloride $\left(\mathrm{HAuCl}_{4}\right)$ and $1 \mathrm{mM}$ hydroxylamine for 10 minutes led to a $50 \mathrm{~nm}$ thick gold film. The surface roughness associated with a $57 \mathrm{~nm}$ thick gold film was $6.8 \mathrm{~nm}$ [26].

One of the latest studies on the electroless gold deposition for SPR substrates was conducted by Lei et al. [24] in 2008. A mixture of $\mathrm{HAuCl}_{4}$ and hydroxylamine was also employed in this study as the plating solution and APTMS was used to form an adhesive layer to anchor $3 \mathrm{~nm}$ diameter colloidal gold nanoparticles. The results indicated thicker gold films were obtained by increasing the plating time. Figure 16 shows the SPR responses of different plating times applied in their study.

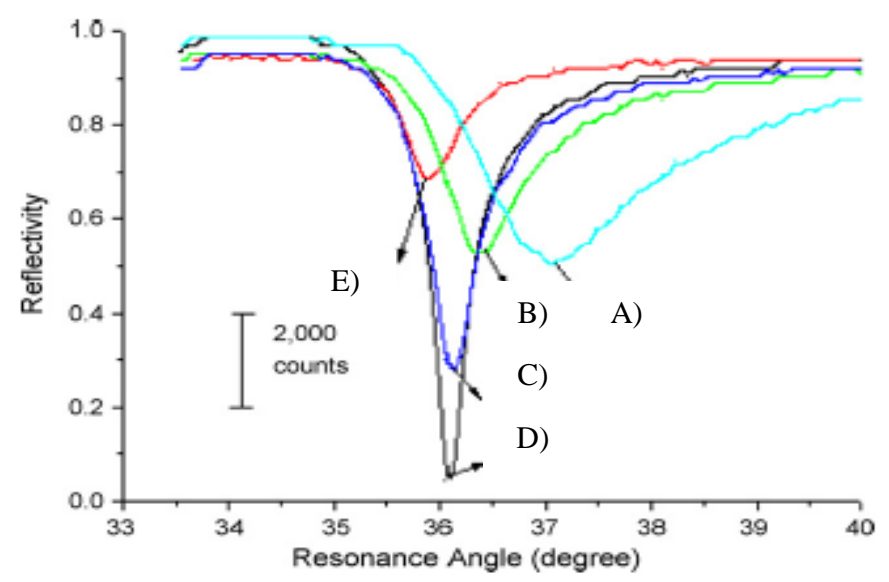

Figure 16. Plating time effects on the SPR responses of gold films grown for: A) 3 min, B) $3.5 \mathrm{~min}, \mathrm{C}$ ) $4 \mathrm{~min}, \mathrm{D}) 4.5 \mathrm{~min}$, and E) 6 min [24] (reprinted with permission from Elsevier). 


\subsection{Electroless Plating to Form 3D Gold Nanoshells}

The synthesis of gold nanoshells was first reported by Oldenburg et al. [46] in 1999. They used $120 \mathrm{~nm}$ silica nanoparticles as the dielectric core material in their study. The silica nanoparticles were synthesized via the Stöber method and functionalized with APTMS to provide amine-termination at the outer side of the silica. Small gold colloids (1-2 $\mathrm{nm}$ in diameter) were added to these aminated silica particles to covalently bond with the amine groups. The small gold colloids were synthesized via gold (III) chloride reduction by tetrakishydroxymethylphosphonium chloride (THPC). The gold-decorated silica particles then served as the nucleation sites (seeds) for the reduction of an aged solution of gold (III) chloride and potassium carbonate by sodium borohydride. The study showed that the reduction led to the enlargement of the gold seeds that eventually formed a continuous gold shell.

Oldenburg et al. [46] used TEM and UV-visible spectrophotometer to closely follow the attachment of gold seeds to the functionalized silica particles and the enlargement process. From the UV-visible spectra, the gold shell formation was indicated by the shifts of the absorbance peak from $550 \mathrm{~nm}$ to $800 \mathrm{~nm}$, as shown in Figure 17. According to Oldenburg et al. [46], the broader characteristic of absorbance peak indicated that the enlargement process had occurred but it had not led to a complete formation of a gold shell [46]. Once the shell formation was completed, a more defined absorbance peak was observed. As the shell became thicker, the absorbance peak at the longer wavelength shifted back to the shorter wavelength. The TEM images that demonstrate the growth process are shown in Figure 18. 


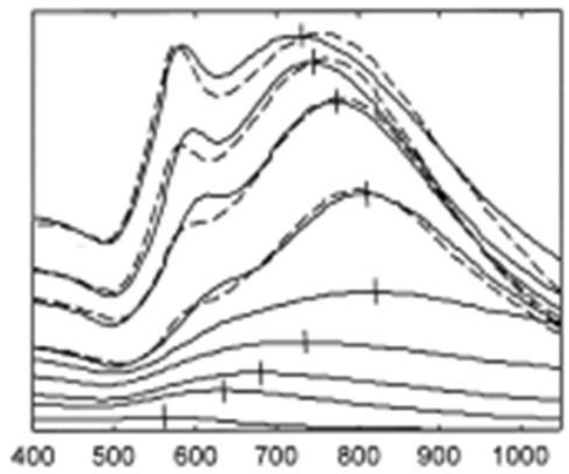

Figure 17. UV-visible spectra depicting the progress of gold nanoshell formation on 120 nm silica particles (lower to upper spectrum) [46] (reprinted with permission from Elsevier).

(a)

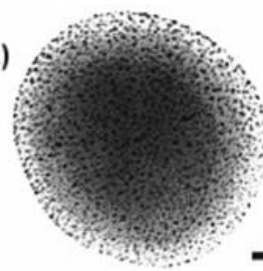

(c)

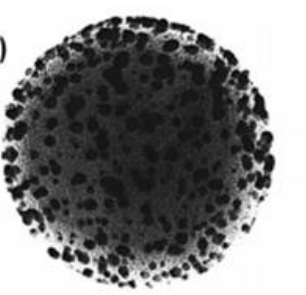

(e)

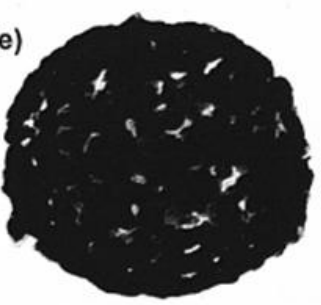

(b)

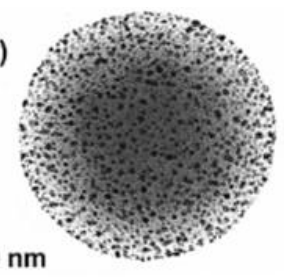

(d)

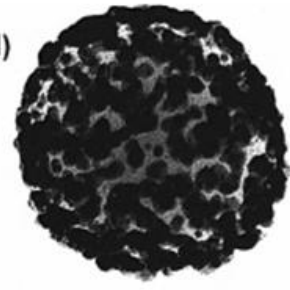

(f)

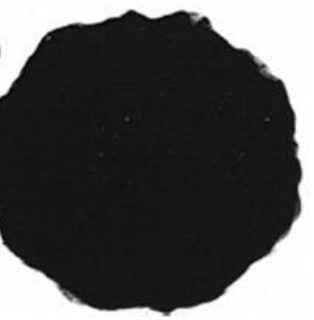

Figure 18. TEM images illustrating the growth process of gold nanoshell formation on $120 \mathrm{~nm}$ silica nanoparticles [46] (reprinted with permission from Elsevier).

The next advancement in gold nanoshell formation was conducted by Westcott and coworkers [67]. They specifically investigated parameters that affected the attachment of gold colloids onto the functionalized silica nanoparticles. The parameters 
they varied were the type of organosilanes used to functionalize the silica nanoparticles and the solvent in which seeding took place. To study the effects of solvent, they used a mixture of water and ethanol at different ratios. Their results revealed that a higher concentration of ethanol led to the aggregation of gold colloids on the silica surface.

As for the investigation of different functional groups, Westcott et al. [67] utilized 3-mercaptopropyltrimethoxysilane (MPTMS), N-(2-aminoethyl)-3aminopropyltrimethoxysilane (AEAPTMS), and 2-(diphenylphosphino) ethyltriethoxysilane (DPPETES). Although all above organosilanes have different functional groups (thiols, amines, and phosphines), these functional groups were known to coordinate with gold due to their lone electron pair availability [67]. Westcott et al. [67] used a mixture of water and ethanol with $\mathrm{pH} 6-8$ in their study. At low $\mathrm{pH}(<10)$ alkylamines exist as $\mathrm{R}^{-\mathrm{NH}_{3}}{ }^{+}$. They suggested that the amines interact via electrostatic interactions with the negatively charged gold colloids (gold/THPC) while thiols and phosphines interact through coordinative interactions. Apart from the interactions between the functional groups of organosilanes (amines, thiols, and phosphines) and gold colloids, their experimental results showed that AEAPTMS (amines) provided a better distribution of gold colloids on the silica's surface compared to MPTMS (thiols) and DPPETES (phosphines). On MPTMS-functionalized and DPPETES-functionalized silica particles, clusters of gold colloids were predominantly observed. This occurrence was observed through TEM analysis, as shown in Figure 19. From this experiment, they also showed that non-functionalized silica did not support the attachment of gold colloids. 


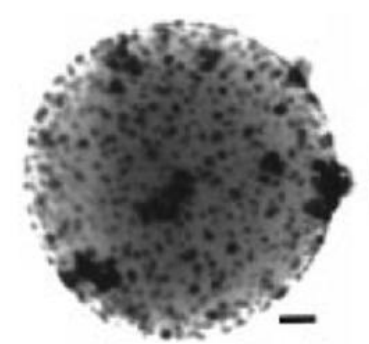

A)

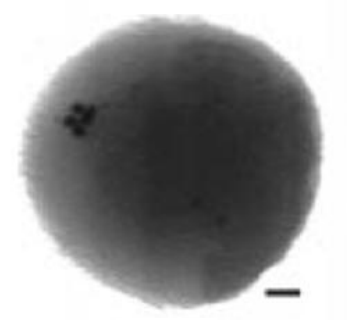

C)

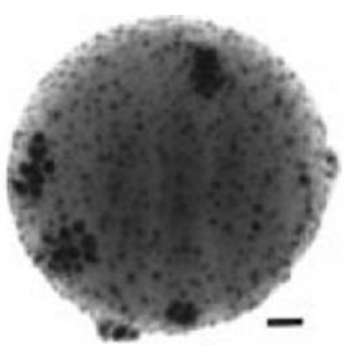

B)

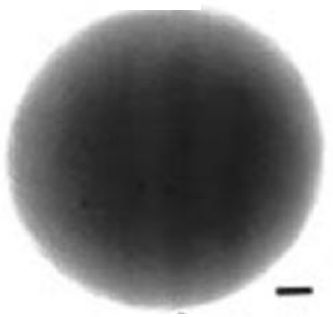

D)

Figure 19. TEM images of the different distributions of gold colloids on silica particles that were functionalized with: A) AEAPTMS, B) MPTMS, C) DPPETES, and D) nonfunctionalized [67] (reprinted with permission from American Chemical Society). Scale bar: $10 \mathrm{~nm}$.

Pham et al. [68] later showed that the typical procedure developed by Oldenburg et al. [46] was robust. They utilized $100 \mathrm{~nm}$ silica nanoparticles as core materials that were then functionalized with APTMS. Gold colloids in the size of $2 \mathrm{~nm}$ (Gold/THPC) were added subsequently to the APTMS-functionalized silica nanoparticles. Multiple centrifugations were performed to remove any unbound gold colloids. Further reduction of gold (III) chloride and potassium carbonate by a reducing agent led to the formation of gold nanoshells on the seeded silica nanoparticles. In their study, Pham et al. [68] used formaldehyde as the reducing agent instead of sodium borohydride as employed by Oldenburg et al. [46]. The functionalization, seeding, and growth steps are illustrated in Figure 20. After the gold nanoshells were formed, Pham et al. [68] further functionalized the gold shells with alkanethiols via self-assembly. They claimed that the self-assembled 
monolayer (SAM) of alkanethiol increased the stability of gold nanoshells to air and moisture. It also allowed gold nanoshells to become soluble in organic solvents.

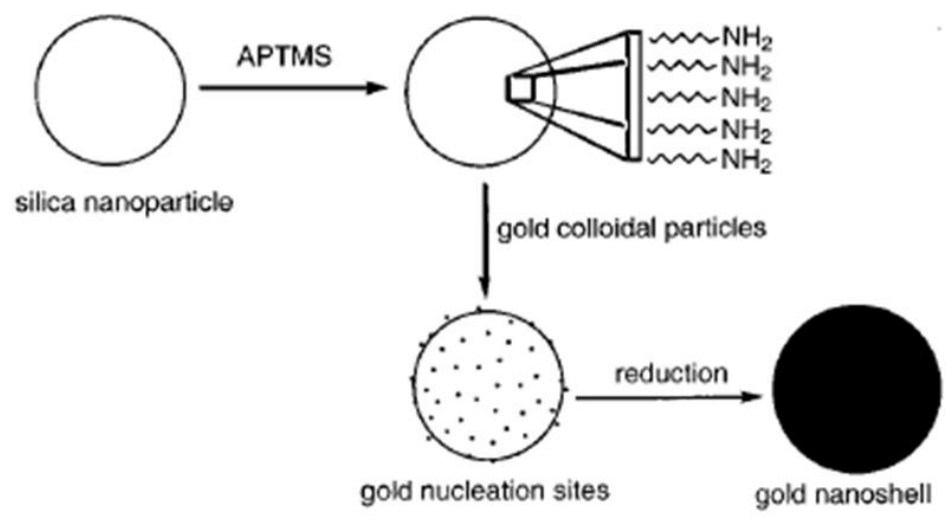

Figure 20. Illustration of gold nanoshell formation on a silica nanoparticle [68].

The use of formaldehyde as an effective reducing agent in the growth step was also demonstrated by Park et al. [69], Ji et al. [19], and Lim et al. [70]. Park et al. [69] specifically investigated the effects of $\mathrm{pH}$ on the formation of gold nanoshells on APTMS-functionalized silica nanoparticles. They utilized 1-3 nm gold/THPC as seeds and adjusted the $\mathrm{pH}$ of this gold/THPC solution by adding $\mathrm{NaOH}$ or $\mathrm{HCl}$ prior to the addition of functionalized silica particles. The gold colloids were found to attach to the functionalized silica particles in a monodisperse fashion at low $\mathrm{pH}$ while at high $\mathrm{pH}$ the gold colloids had a tendency to form clusters, as shown in Figure 21. According to Park et al. [69], the monodisperse deposition of gold colloids led to smoother gold nanoshells. As a result, a stronger absorption peak was observed. 


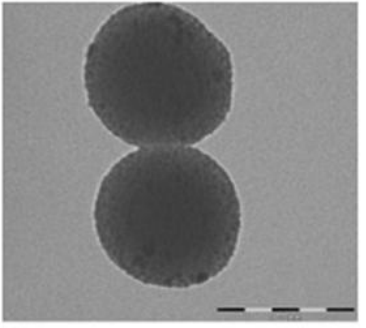

A)

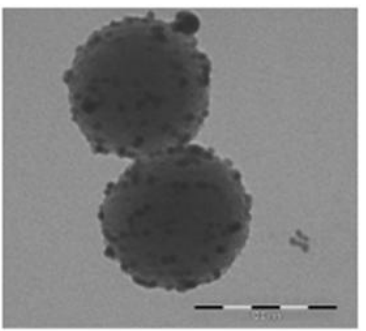

C)

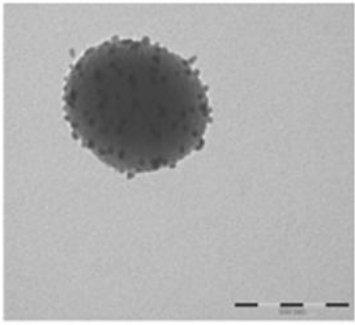

B)

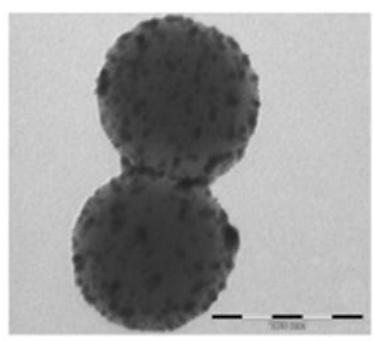

D)

Figure 21. TEM images of colloidal gold attachment on the APTMS-functionalized silica particles (core diameter: $80 \mathrm{~nm}$ ) at: A) $\mathrm{pH} 3.1, \mathrm{~B}$ ) $\mathrm{pH} 7.0, \mathrm{C}$ ) $\mathrm{pH} 9.7$, and D) $\mathrm{pH}$ 10.3 of gold colloids solution [69] (reprinted with permission from The Korean Chemical Society).

In addition to sodium borohydride and formaldehyde, some researchers demonstrated that hydroxylamine hydrochloride was also a good reducing agent in promoting the formation of gold nanoshells. According to Graf and Blaaderen [18], hydroxylamine hydrochloride is a strong reducing agent that enables uniform growth rate on the nucleation sites. They hypothesized that a well-defined, closely packed, and monodisperse nanoshell could be obtained with a more uniform growth rate. In their study, silica particles with a $205 \mathrm{~nm}$ radius were utilized as core materials and functionalized with APTMS prior to the gold seed deposition. The typical 1-3 nm gold colloids that were synthesized from the reduction of gold (III) chloride by THPC were used as seeds. After the exposure with gold hydroxide and hydroxylamine hydrochloride, gold nanoshells were formed on these silica cores, as shown in Figure 22. 


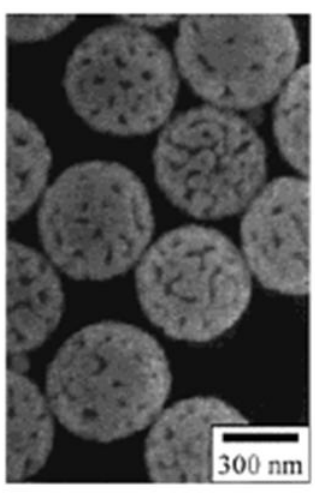

A)

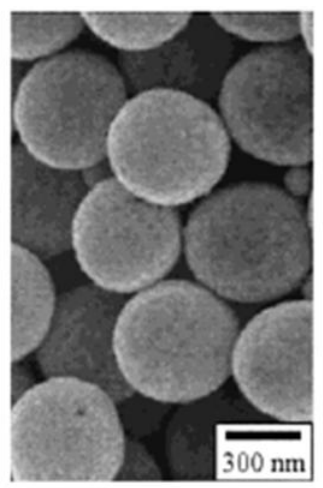

B)

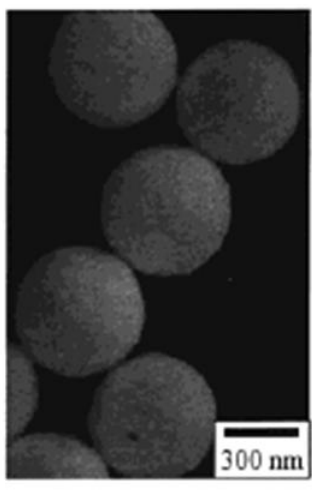

C)

Figure 22. SEM images of silica nanoparticies that were coated with: A) $23 \mathrm{~nm}$, B) 39 $\mathrm{nm}$, and C) $63 \mathrm{~nm}$ gold shell [18] (reprinted with permission from American Chemical Society).

Graf and Blaaderen [18] also verified the ability to perform multiple growth steps to attain a certain shell thickness. First, they synthesized a thin gold shell $(39 \mathrm{~nm})$ on silica cores and used these thin gold nanoshells as seeds for the second growth. The second growth resulted in a thicker gold shell $(88 \mathrm{~nm})$ with a relatively low polydispersity, as shown in Figure 23. This approach can be used to form bimetallic structures by growing silver on top of the gold shell.

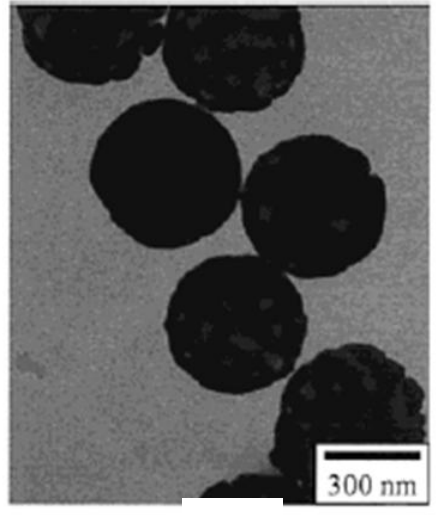

A)

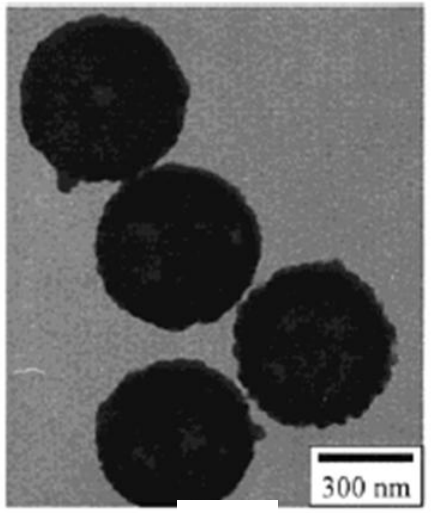

B)

Figure 23. TEM images of silica particles with: A) a $39 \mathrm{~nm}$ gold shell and B) a $82 \mathrm{~nm}$ gold shell that were obtained through the second growth step of the silica particles with $39 \mathrm{~nm}$ gold shell [18] (reprinted with permission from American Chemical Society). 
Shi and coworkers [16] also verified the efficacy of hydroxylamine hydrochloride as a reducing agent in the gold shell formation. They employed $\mathrm{COOH}$-modifiedpolystyrene instead of the typical silica particles as the core material for the gold nanoshell formation. The routine procedures of functionalization, seeding, and growth were again applied in their experiments. For the functionalization, they mixed the COOH-modified-polystyrene with 2-aminoethanethiol hydrochloride (AET) and N-ethylN'-(3-(dimethylamino)propyl)carboiimide hydrochloride (EDAC). This reaction resulted in $\sim \mathrm{SH}$-terminated polystyrene. Addition of $2 \mathrm{~nm}$ Gold/THPC to the $\sim \mathrm{SH}$-terminated polystyrene produced gold-decorated or gold-seeded polystyrene. With the reduction of gold hydroxide by hydroxylamine hydrochloride, the seeded polystyrenes were finally coated with gold nanoshells. This transformation is illustrated by TEM images in Figure 24 (left). Red-shifts in absorbance peak of these transformed particles were also observed in Figure 24 (right). A thicker shell led to a longer wavelength absorbance.
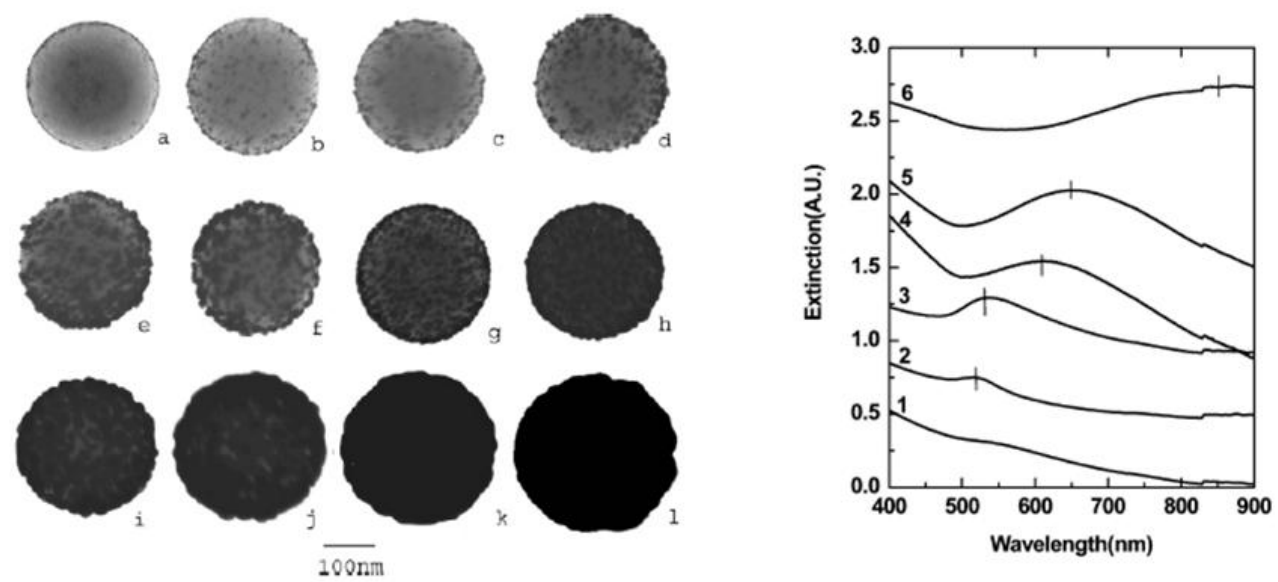

Figure 24. TEM images of gold nanoshell formation in a progressive order (left). Redshifts in the absorbance peaks as the gold nanoshells get thicker (right). Curve 1 in the right image corresponds to a-b, curve 5 corresponds to g-i, and curve 6 corresponds to $\mathrm{j}-\mathrm{k}$ in the left image [16] (reprinted with permission from American Chemical Society). 
Further advancement in the gold nanoshell formation was achieved by Lee et al. [20]. Instead of typical silica/gold core-shell materials, they incorporated magnetic kernels that were composed of $\mathrm{MnFe}_{2} \mathrm{O}_{4}$ as the main core. Magnetic kernels allow the magnetic gold composites to act as MRI contrast agents and photothermal ablation agents. Their approach is illustrated in Figure 25.
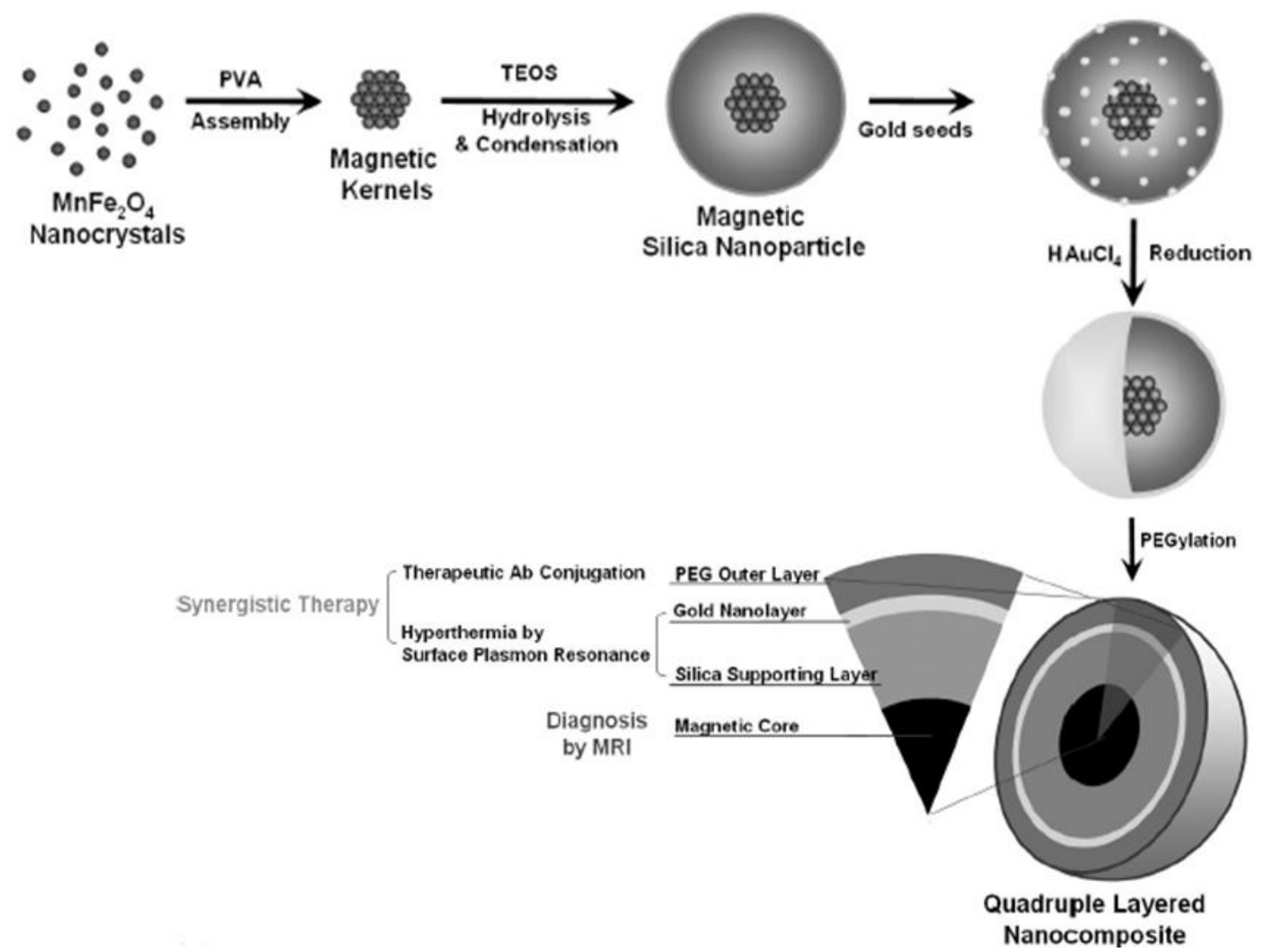

Figure 25. Schematic of magnetic gold nanocomposite synthesis [20]. Copyright WileyVCH Verlag GmbH \& Co. Reproduced with permission.

Lee et al. [20] started their process by forming magnetic kernels through polyvinyl alcohol (PVA)-assisted self-assembly of $\mathrm{MnFe}_{2} \mathrm{O}_{4}$. Silica encapsulation of these magnetic kernels via the Stöber method followed. The silica particles were grown to a final size of $\sim 100 \mathrm{~nm}$ in diameter. Further functionalization of silica particles by 
APTMS was conducted to provide attachment sites for gold colloid deposition. Once small gold colloids (Gold/THPC) were attached to the silica particles, the growth step was initiated by adding a mixture of gold hydroxide and hydroxylamine. To make these magnetic gold nanocomposites biocompatible, PEGylated was performed to have polyethylene glycol (PEG) as the outer layer. Their experimental results showed that after the gold shells were formed, a characteristic absorbance at the NIR window was observed and blue-colored solution was obtained, as shown in Figure 26.
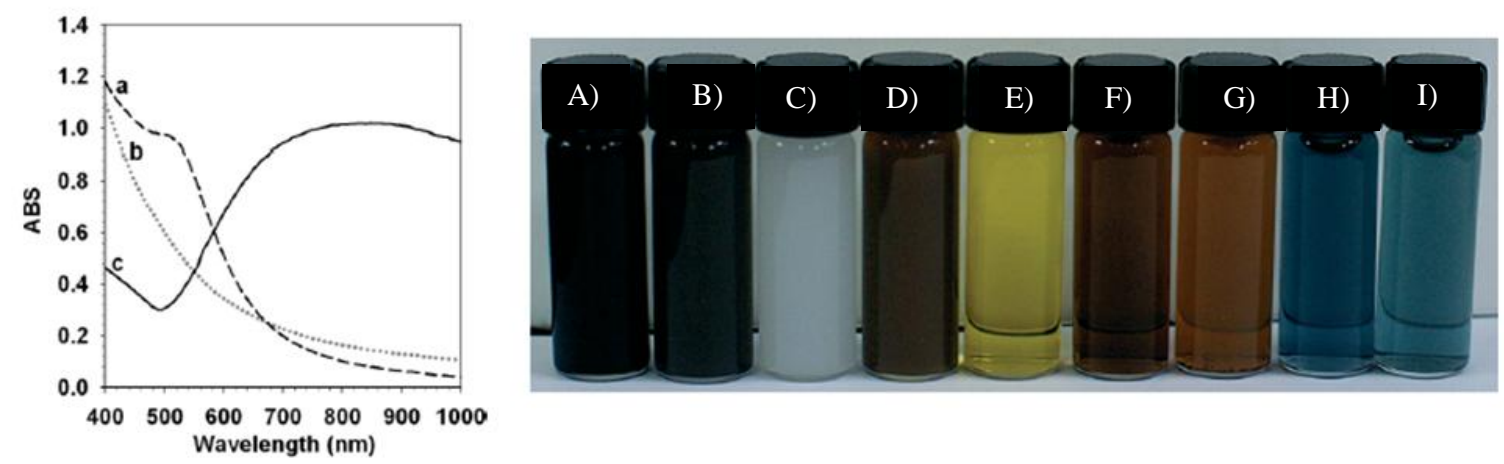

Figure 26. Absorption at the NIR window ( $~ 850 \mathrm{~nm}$ in this case) was observed after the formation of gold shell (left). The right figure shows the solution color of: A) $\mathrm{MnFe}_{2} \mathrm{O}_{4}$ in hexane, B) magnetic kernels, C) silica nanoparticles (NPs), D) magnetic silica NPs, E) gold (III) chloride, F) gold/THPC, G) magnetic silica NPs-gold/THPC, H) magnetic gold nanocomposites (MGNCs), and I) PEGylated MGNCs in water [20]. Copyright WileyVCH Verlag $\mathrm{GmbH} \& \mathrm{Co}$. Reproduced with permission.

\subsection{Summary}

Studies have shown that hyperbranched polymers such as dendrimers and star polymers can be deposited as multilayer films [53-58]. In particular, star polymers have been demonstrated to form self-assembled multilayer films that were uniform and stable $[57,58]$. The appropriate functionalized star polymers should provide similar interaction capabilities as organosilanes. In addition, according to Crooks et al. $[42,71]$, the three- 
dimensional form of hyperbranched polymers should provide a larger number of attachment sites that correlates to better adhesion and stability compared to small molecules such as organosilanes.

There are three main features that can be inferred from the current literature on electroless gold deposition on glass substrates. First, an adhesive layer, which is commonly built from organosilane compounds, is required to anchor colloidal gold particles. Second, a surface-confined colloidal gold should be available as a seed or catalytic site. A dense monolayer of relatively small gold nanoparticles ( $3 \mathrm{~nm}$ in diameter) was shown to produce a uniform and contiguous gold film [24]. The third feature is the need for a proper growth or plating solution that enlarges the gold seed without creating new nucleation sites. The mixture of hydroxylamine hydrochloride and gold (III) chloride has been shown to be an effective solution in several studies [25, 26, 41]. Concentration of the growth solution, plating time and agitation also had significant effects on plated gold film thickness and uniformity [24, 26].

Similar steps (templating, seeding, and plating) were also observed in the 3D gold nanoshell formation $[16,18,20,46,67-70]$. Silica nanoparticles were the most common core materials and APTMS was the most commonly used material to functionalize these silica particles with amine groups [20, 46, 69]. 1-3 nm gold colloids that acted as seeds were attached to the amine groups on the outer side of silica cores. These 1-3 nm gold colloids were typically synthesized through gold (III) chloride reduction by tetrakishydroxymethylphosphonium chloride (THPC) [16, 20, 46, 67-69]. Subsequently, multiple centrifugation/redispersion was required to remove any unbound gold colloids 
that could create non-templated gold nanoshells $[16,68]$. The mixture of hydroxylamine hydrochloride and gold hydroxide was demonstrated to be an effective growth solution to grow thin gold shells from the seeded silica particles $[16,18,20]$. The progress of gold nanoshell formation was typically characterized through TEM and UV-visible spectrophotometer. A red-shift in the absorbance peak was one indication that gold nanoshells had been formed [16, 20,46]. Visually, a blue-colored solution as a result of the NIR absorption was used as an indicator that gold nanoshells had been synthesized [20]. 


\section{CHAPTER THREE}

\section{HYPOTHESIS AND OBJECTIVES}

\subsection{Hypothesis and Justification}

The hypothesis of this study was that star polymers could be used as templates in the formation of 2D thin gold films and 3D gold nanoshells via electroless plating. Since amino groups appear both on amino-functionalized star polymers and aminosilane compounds, star polymers should depict similar capabilities as aminosilanes in enabling a self-assembled gold seed layer via electrostatic interactions. In addition, according to Crooks et al. $[42,71]$, the three-dimensional form of hyperbranched polymers should provide a larger number of attachment sites, which correlates to better adhesion and stability compared to small molecules such as organosilanes.

The objectives of this study were to produce a reproducible and stable thin gold film on glass substrates via electroless plating by utilizing self-assembled star polymers as templates, and to characterize the plated gold film by AFM, UV-visible spectrophotometer, SPR, RBS, and nanoindentation. The electroless plating process conditions were optimized to target $50 \mathrm{~nm}$ gold films. The other objectives were to produce star polymer-templated 3D gold nanoshells with a controllable size and NIR absorption and to characterize the particles by UV-visible spectrophotometer, SEM, TEM, and electron energy loss spectroscopy (EELS). 


\subsection{Motivation}

Surface plasmon resonance (SPR) sensors have drawn considerable interest since it offers a tagless, highly sensitive, and real-time detection. A thin metal film (gold is the common metal) is necessary to build an SPR substrate. Simple, efficient, and solutionbased electroless plating allows SPR substrate preparation in any wet chemical laboratory without any instrumental constraints. The ability to use different adhesive layers for promoting thin gold film growth via electroless plating supports process robustness. The simplicity in substrate preparation leads to increased usages of SPR sensors. In addition, the ease in incorporating other materials such as dyes and drugs into the interstitial space of star polymer makes star polymer-templated gold nanoshells a promising candidate for integrated imaging, photothermal ablation, and drug-delivery agents in cancer imaging and therapy. 


\section{CHAPTER FOUR}

\section{MATERIALS AND METHODS}

This chapter describes the experimental setup used to determine the acceptable operating parameters to achieve $2 \mathrm{D}$ and $3 \mathrm{D}$ gold nanostructures. As described in the literature review chapter, there are four main parameters in 2D electroless gold plating that can be optimized to get a specified film thickness. These four main factors include growth/plating solution concentration, plating time, agitation, and seed distribution. In the formation of 2D gold films, the seeds were synthesized according to Lei et al.'s [24] procedure. The seed size from this procedure was expected to be approximately 3-5 nm in diameter. Seed distribution refers to the seed coverage on star polymer-templated substrates. The optimum seeding time that gave full coverage was determined by varying seeding time. The coverage process was monitored by AFM and UV-visible spectrophotometer.

Agitation of the plating solution at $65 \mathrm{rpm}$ was provided by a circular shaker. The effects of seeding time, plating time, and plating solution concentration on thin gold film formation were studied. However, the first experiments were dedicated to determine the process conditions which formed a thin star polymer film as an adhesive layer for gold seed confinement. According to Bonifacio and Chang $[57,58]$, the formation of star polymer films was feasible in minutes. In this project, star polymer film deposition time was set at 30 minutes to ensure a good film formation. The concentration of the star polymer solution was constant. The design of main experiments that were carried out to form 2D gold films via electroless deposition is presented in Table 1. 
Table 1. The design of main experiments to form electroless $2 \mathrm{D}$ gold films.

\begin{tabular}{|c|c|c|c|}
\hline Step & $\begin{array}{l}\text { Type of } \\
\text { experiments }\end{array}$ & Parameters & Characterizations \\
\hline Templating & $\begin{array}{l}\text { Star PS-DMAEMA } \\
\text { deposition }\end{array}$ & $\begin{array}{l}\text { - Type of substrates } \\
\text { (silicon wafer and glass) } \\
\text { - Solvent (water and } \\
\text { toluene) } \\
\text { - Dipping time }\end{array}$ & $\begin{array}{l}\text { AFM and } \\
\text { SPR spectroscopy }\end{array}$ \\
\hline \multirow[b]{2}{*}{ Seeding } & Gold seed synthesis & Reagent concentration & UV-visible and TEM \\
\hline & $\begin{array}{l}\text { Seed attachment to } \\
\text { star polymer layer }\end{array}$ & Seeding time & $\begin{array}{l}\text { UV-visible, AFM, } \\
\text { and SPR }\end{array}$ \\
\hline \multirow{3}{*}{ Growth } & Thickness control & $\begin{array}{l}\text { Growth time and growth } \\
\text { solution concentration }\end{array}$ & $\begin{array}{l}\text { Alpha step, RBS, } \\
\text { AFM, SPR, and UV- } \\
\text { visible }\end{array}$ \\
\hline & Film uniformity & Method of agitation & RBS \\
\hline & $\begin{array}{l}\text { Comparison of star } \\
\text { polymer with } \\
\text { APTMS and PAH } \\
\text { in promoting gold } \\
\text { growth }\end{array}$ & $\begin{array}{l}\text { - Type of adhesive } \\
\text { materials (star, APTMS, } \\
\text { and PAH) } \\
\text { - Templating and seeding } \\
\text { time }\end{array}$ & $\begin{array}{l}\text { RBS, AFM, and } \\
\text { Nanoindentation } \\
\text { (Scratch tests) }\end{array}$ \\
\hline
\end{tabular}

In terms of 3D gold nanoshell formation, prior investigations indicated that small

gold colloids (1-3 nm) were required to be attached to the functionalized core materials to act as seeds. Therefore, a different method to synthesize small gold colloids was

employed. THPC was used as the reducing agent instead of sodium borohydride as used in the seed synthesis for 2D gold film experiments since THPC resulted in smaller gold colloids $(1-3 \mathrm{~nm})$ compared to sodium borohydride $(5 \mathrm{~nm})$. The concentration of the gold/THPC stock solution was maintained constant throughout experiments. The amount of gold/THPC that was added to the star polymer solution was varied by adjusting the volume of the gold/THPC stock solution that was added to the reaction mixture. The 
growth solution was comprised of gold hydroxide and hydroxylamine hydrochloride as employed by previous researchers. Gold hydroxide was obtained by reacting gold (III) chloride and potassium carbonate. The growth solution concentration was varied to achieve the desirable particle size. The growth time was also adjusted to generate monodisperse particles. The experimental matrix for the 3D gold nanoshell research is summarized in Table 2.

Table 2. The design of experiments to form 3D gold nanoshells with star polymer as the core material.

\begin{tabular}{|l|l|l|l|}
\hline Step & Type of experiments & Parameters & Characterizations \\
\hline \multirow{2}{*}{ Seeding } & Gold seed synthesis & Reagent concentration & UV-visible \\
\cline { 2 - 4 } & $\begin{array}{l}\text { Seed attachment to } \\
\text { star polymer }\end{array}$ & Seed amount & UV-visible \\
\hline Growth & $\begin{array}{l}\text { Thickness/size } \\
\text { control }\end{array}$ & $\begin{array}{l}\text { Growth time and } \\
\text { growth solution } \\
\text { concentration }\end{array}$ & $\begin{array}{l}\text { UV-visible, SEM, and } \\
\text { EELS }\end{array}$ \\
\hline
\end{tabular}

\subsection{Materials}

Star polymers with a polystyrene core and amino-functionalized peripheries were synthesized based on IBM procedures developed by Sly et al. [43]. The star polymers used in this study had approximately 28 arms and a $21 \mathrm{~nm}$ hydrodynamic diameter. The molecular weight based on nuclear magnetic resonance (NMR) spectroscopy was 252,000 Daltons (Da).

Gold (III) chloride ( $\mathrm{HAuCl}_{4}$.hydrate), trisodium citrate, sodium borohydride $\left(\mathrm{NaBH}_{4}\right)$, potassium carbonate, tetrahydrofuran (THF), tetrakishydroxymethylphosphonium chloride (THPC), and hydroxylamine hydrochloride 
$\left(\mathrm{NH}_{2} \mathrm{OH} . \mathrm{HCl}\right)$ were used as purchased from Sigma-Aldrich. All chemicals were analytical grade and used without further purification. Millipore water was the main solvent used. It was obtained from the Millipore filter unit at IBM Almaden Research Center (IBM ARC). Millipore water is chosen because it goes through a very fine purification process, which minimizes any ions or organic compounds.

The SPR instrument at IBM ARC utilizes a prism that is made of SF 11 materials. Thus, to make it compatible, SF 11 substrates with $1 \mathrm{~mm}$ thickness and 1 inch diameter were used. These SF 11 substrates were purchased from Stefan Sydor Optics. Several preliminary experiments, such as the star polymer deposition were carried out using quartz wafers instead of SF 11. Quartz wafers are chosen because they have similar physical and chemical properties to SF 11. Quartz wafers with one inch diameter and 0.5 mm thickness were purchased from Boston Piezo-Optics.

\subsection{Methods}

\subsubsection{Quartz and SF 11 Surface Treatment}

Prior to use, quartz and SF 11 substrates were cleansed of any organic impurities using a piranha solution treatment. Piranha solution consists of a mixture of sulfuric acid and hydrogen peroxide. In this study, a mixture of $80 \% \mathrm{v} / \mathrm{v}$ sulfuric acid and $20 \% \mathrm{v} / \mathrm{v}$ hydrogen peroxide was used. The treatment involved substrate immersion into piranha solution for 30 minutes, followed by a water rinse. Piranha solution should be handled with extra caution since it is extremely corrosive.

After the piranha solution treatment, quartz or SF 11 substrates were exposed to a mixture of $80 \% \mathrm{v} / \mathrm{v}$ ammonium hydroxide and $20 \% \mathrm{v} / \mathrm{v}$ hydrogen peroxide. This 
treatment enriches the quartz or SF 11 surface with hydroxyl groups that are crucial in the binding process of star polymers to a SF 11 or quartz surface. After a 30 minutes exposure to the ammonium hydroxide/hydrogen peroxide mixture, the substrates were rinsed using ethanol and then dried with nitrogen flow.

\subsubsection{Star Polymer Solution Preparation for 2D Gold Films}

The star polymers used in this project were obtained in a powder form. The star polymer solution was prepared by dissolving $20 \mathrm{mg}$ of star polymers in $1 \mathrm{~mL} \mathrm{THF}$. After the star polymer was dissolved in THF, $4 \mathrm{~mL}$ of Millipore water was added drop-wise to the mixture of star polymer/THF. THF was added as an intermediate solvent to accelerate the dissolving process of star polymers in water. To eliminate any impurities, this star polymer solution was passed through a $0.2 \mu \mathrm{m}$ nylon filter before it was being deposited onto quartz or SF 11 substrates.

\subsubsection{Star Polymer Solution for 3D Gold Nanoshells}

Free-base (FB) 5,10,15,20-(3-5-di-tertbutylphenyl) porphyrins were incorporated inside the star polymer to provide insight of the fluorescence enhancement by gold nanoshells. The preparation of star polymer/porphyrin occlusion complexes was started by dissolving $10 \mathrm{mg}$ of star polymer in $1 \mathrm{~mL}$ of THF. Subsequently, $2 \mathrm{mg}$ of FB porphyrin was added to the star polymer/THF solution. This mixture was passed through a $0.2 \mu \mathrm{m}$ Teflon filter and added drop-wise to $4 \mathrm{~mL}$ of Millipore water. Due to the porphyrins insolubility in water, porphyrins are driven to the hydrophobic interstitial 
space of the star polymers. The final solution of star polymer/porphyrin complexes was passed through a $0.45 \mu \mathrm{m}$ glass filter prior to the seeding process.

\subsubsection{Citrate-capped Colloidal Gold Synthesis (for 2D Gold Films)}

The colloidal gold used for seed purposes in the $2 \mathrm{D}$ gold film experiments was synthesized based on Lei et al.'s [24] method. A $0.1 \%$ w/v trisodium citrate was added to a $0.01 \% \mathrm{w} / \mathrm{v}$ aqueous $\mathrm{HAuCl}_{4}$ with a vigorous stirring for one minute. After adding trisodium citrate, a $0.075 \% \mathrm{w} / \mathrm{v}$ of $\mathrm{NaBH}_{4}$ was added to the mixture. Vigorous stirring was continued for another five minutes after the $\mathrm{NaBH}_{4}$ addition. As soon as $\mathrm{NaBH}_{4}$ was added, the mixture exhibited a clear pinkish color. Gold colloids with an average of 3-5 $\mathrm{nm}$ in diameter were expected from this procedure. The size of the synthesized gold colloids was verified using UV-visible spectrophotometer and TEM. According to Lei $e t$ al. [24], gold colloids with $3 \mathrm{~nm}$ diameter should have a UV-visible signature peak at 506 nm. The UV-visible absorbance peak for gold nanoparticles depends heavily on particle size. The larger the particles, the more UV-visible peak shifts towards a longer wavelength.

\subsubsection{Gold/THPC Synthesis (for 3D Gold Nanoshells)}

Pham et al.'s [68] method of fabricating 1-3 nm gold colloids through reduction of gold (III) chloride by THPC was used. In summary, $4.5 \mathrm{~mL}$ of $\mathrm{NaOH} 0.2 \mathrm{M}$ was added into $45.5 \mathrm{~mL}$ of Millipore water and solution was stirred for 2 minutes at $600 \mathrm{rpm}$. Subsequently, $12 \mu \mathrm{L}$ of $80 \%$ THPC that was diluted in $1 \mathrm{~mL}$ of Millipore water was added to the mixture and stirred for another 2 minutes. The $\mathrm{pH}$ of the solution was in the 
range of 12 as suggested by literature. The final step was the fast addition of $2 \mathrm{~mL}$ of gold (III) chloride $0.029 \mathrm{M}$ then the solution was stirred for another 5 minutes. The color of the solution changed from colorless to light brownish as soon as gold (III) chloride was added. This indicated the formation of 1-3 nm gold colloids as reported by Shi et al. [16].

\subsubsection{Plated Gold Film Preparation}

After SF 11 or quartz substrates were treated with the piranha solution and the ammonium hydroxide/hydrogen peroxide mixture, the substrates were dipped in a star polymer solution for 30 minutes and then rinsed with Millipore water to remove any unbound star polymer material. The star polymer-coated substrates were then ready for the seeding step. The seeding process was performed by immersing the coated substrates in the gold colloid solution for one hour. These substrates were then rinsed with Millipore water and dried in a nitrogen flow. As shown by Jin et al. [41], the binding process of gold colloids onto a modified substrate changed the substrate color from transparent to pink. To further verify gold colloid attachments, UV-visible and AFM analysis were conducted.

A plating solution was prepared from a mixture of $\mathrm{HAuCl}_{4}$ and hydroxylamine hydrochloride. The $0.1 \%$ plating solution consisted of $0.1 \% \mathrm{w} / \mathrm{v}^{\mathrm{HAuCl}}{ }_{4}(5 \mathrm{mg} / 5 \mathrm{~mL}$ Millipore water) and $347.5 \mathrm{mg} \mathrm{NH} \mathrm{N}_{2} \mathrm{OH} . \mathrm{HCl}$ in $5 \mathrm{~mL}$ of Millipore water. Plating solution concentrations of $0.2 \%$ and $0.5 \%$ were prepared by proportioning the $0.1 \%$ solution recipe. The plating process began when a seeded substrate was dipped into the plating solution. A color change from pink to blue to gold luster was observed as described by 
Jin et al. [41]. Substrates that were coated with a thin gold film were then rinsed with an excessive amount of Millipore water and dried in a stream of nitrogen. Gold film thickness was analyzed by Rutherford backscattering (RBS) method while gold film morphology, roughness, and stability were analyzed by AFM. SPR spectroscopy further verified the presence of any plasmon resonance effect of the plated gold films.

\subsubsection{D Gold Nanoshell Synthesis}

For seeding process, $5 \mathrm{~mL}$ of star polymer/porphyrin solution was mixed with 5 $\mathrm{mL}$ of freshly prepared gold/THPC. This solution was diluted with $10 \mathrm{~mL}$ of Millipore water and stirred overnight. After overnight stirring, the solution was dialyzed against Millipore water using a cellulose dialysis membrane with 12-14,000 Da molecular weight cut-off (MWCO) for 24 hours. The purpose of the dialysis was to remove any excess reagents or by-product salts from the gold/THPC solution. The dialysed solution was stored at $4{ }^{\circ} \mathrm{C}$ prior to its use as the seed solution in the growth step.

As mentioned previously, a mixture of gold hydroxide and hydroxylamine hydrochloride was chosen to be used as the growth solution in the gold nanoshell experiments. Gold hydroxide was attained by adding $100 \mathrm{mg}$ potassium carbonate into a solution of $1.74 \mathrm{~mL}$ of gold (III) chloride $(0.0955 \mathrm{M})$ that was diluted with $98.2 \mathrm{~mL}$ of Millipore water. Gold hydroxide was dark-aged for 24 hours prior to use. A freshly prepared hydroxylamine hydrochloride $0.026 \% \mathrm{w} / \mathrm{v}$ was used as the reducing agent solution.

The growth step was initiated by mixing a specified volume of gold hydroxide and seed solution (star/porphyrin-gold/THPC) at $650 \mathrm{rpm}$ for one minute. A specified 
volume of hydroxylamine hydrochloride solution was then added to the mixture. The volume ratio of gold hydroxide, seed solution, and hydroxylamine hydrochloride depends on the desired size of final particles. For instance, to achieve an average of $100 \mathrm{~nm}$ particles (in diameter), the volume of gold hydroxide, seed solution, and hydroxylamine hydrochloride was $14.8 \mathrm{~mL}, 9 \mathrm{~mL}$, and $20 \mathrm{~mL}$, respectively. The final solution was stirred for another 15 minutes and stored at $4{ }^{\circ} \mathrm{C}$ prior to further purification steps.

In order to have 'clean' particles for SEM imaging, the gold nanoshell solution was dialysed against water for 24 hours and then against methanol for 24 hours. Both dialysis steps utilized a cellulose membrane with 12-14,000 Da MWCO. The dialysed solution was then spin-coated onto a clean silicon wafer for 30 seconds at 1,000 rpm. The wafer was then heated at $90{ }^{\circ} \mathrm{C}$ for 1 minute to remove any remaining solvent and cooled down to room temperature prior to SEM imaging.

\subsection{Instrumentation}

\subsubsection{Circular Shaker}

A VWR DS-500E digital circular shaker was used as the agitation provider for the plating process. This shaker operates in a circular motion that travels over a $19 \mathrm{~mm}$ orbit. The speed range is $25-500 \mathrm{rpm}$, however, for this study the agitation rate was set at a constant rate of $65 \mathrm{rpm}$. A constant rate of $120 \mathrm{rpm}$, as suggested by Lei et al. [24], was initially used in this work. The $120 \mathrm{rpm}$, however, was found to be too fast for the experimental setup. Instead, a constant rate of $65 \mathrm{rpm}$ was shown to be the optimum agitation rate in this study. 


\subsubsection{UV-visible and Fluorescence Spectrophotometer}

An Agilent UV-visible spectrophotometer was employed to verify the gold colloid formation (both citrate-capped and gold/THPC) and the gold colloid attachments onto a star polymer-coated substrate or star polymers in solution. Different holders can be attached to the UV-visible spectrophotometer, thus analysis is made possible for both solution and solid/wafers. According to Lei et al. [24], gold colloids with $3 \mathrm{~nm}$ diameter should have a UV-visible signature peak at $506 \mathrm{~nm}$. Small gold colloids (1-3 nm) exhibit a weak absorbance peak at around $500 \mathrm{~nm}[72]$. In a surface-confined form, the absorbance peak shifts to a longer wavelength $(600-800 \mathrm{~nm})$ [59].

A Fluorolog® Jobin Yvon-Spex from Instruments S.A. Inc. was used to record the fluorescence spectrum of porphyrin-embedded star polymers. The excitation wavelength was set at $420 \mathrm{~nm}$ and the scan range was set at $500-800 \mathrm{~nm}$. This scan range was adjusted to the typical emission peaks of porphyrin at $650 \mathrm{~nm}$ and $720 \mathrm{~nm}$.

\subsubsection{AFM}

A Digital Instruments 3100 AFM with a $1 \mathrm{~N} / \mathrm{m}$ spring constant silicon nitride cantilever was operated in a tapping mode to analyze star polymer films, seeded films, and plated gold films. The scan rate was set at $1 \mathrm{~Hz}$ while scan size was varied from 500 $\mathrm{nm} \times 500 \mathrm{~nm}$ to $5 \mu \mathrm{m} \times 5 \mu \mathrm{m}$ to provide a thorough analysis. A $5 \mu \mathrm{m} \times 5 \mu \mathrm{m}$ area provided information on film coverage while a $500 \mathrm{~nm}$ x $500 \mathrm{~nm}$ area offered information on how individual particles arranged themselves into a surface-confined form. Surface roughness was determined from the RMS (root mean square) roughness analysis that is featured in the Digital Instruments software. 


\subsubsection{SPR}

The SPR used in this study was designed and built by IBM ARC. It is operated by manipulating two arms that act as a source arm and a collecting arm. An $854 \mathrm{~nm}$ laser diode, polarizer, and compensation optics are mounted on the source arm, while receiver optics are mounted on the collection arm to detect the reflected light. These two arms counter rotate at an equal angle of incidence with a 0.001 degree resolution. In the middle section of these two operating arms, a flow cell is placed. An SPR substrate can be positioned on top of the flow cell where the gold side of the substrate faces the flow cell. A vacuum line, connected to the flow cell, holds the substrate in place using a vacuum force. A small amount of an appropriate refractive index matching fluid is added to the uncoated side of the substrate before a SF 11 hemi-cylindrical prism is slowly set on top of the SPR substrate. Air bubbles must be avoided within the index matching fluid, since air trapped at the interface of the SPR substrate and prism will likely cause SPR response interference. A photograph of the SPR instrument at IBM ARC is presented in Figure 27.

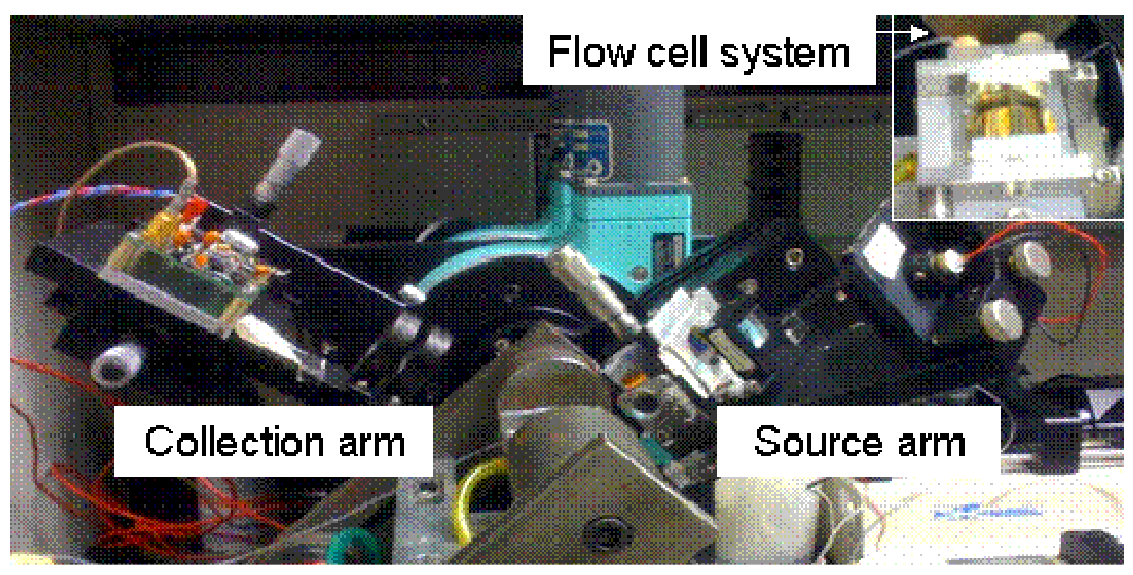

Figure 27. SPR instrument at IBM ARC. The inset is the flow cell system that consists of a flow cell, SPR substrate (surface detector), and SF 11 hemi-cylindrical prism. 


\subsubsection{RBS, SEM, TEM, and EELS}

Rutherford backscattering (RBS) spectroscopy is often used to provide elemental analysis. This technique also provides layer thickness measurement with high accuracy, thus, it is chosen to be the prime instrument for measuring film thickness in this work. Specifically the RBS was performed by Andrew Kellock at IBM ARC using an NEC 3UH Pelletron, using $\mathrm{He}^{+}$ions with a beam current of $20 \mathrm{nA}$ at $2.3 \mathrm{MeV}$. Data reduction and peak fitting were performed using RUMP software.

TEM was required to verify the morphology of the citrate-capped gold colloids (seeds for 2D gold films) while EELS was required to verify the existence of star polymers inside the 3D gold nanoshells. Samples were sent to the TEM/EELS facilities at the IBM ARC, and analysis was performed by Leslie Krupp and Phillip Rice (a Topcon 002B TEM running at $200 \mathrm{kV}$ was used). A Hitachi S-4700 cold field emission SEM was used to verify the morphology of 3D gold nanoshells.

\subsubsection{Nanoindenter}

Scratch tests by Hysitron TI 950 Triboindenter (with a diamond-based Berkovich tip) were performed to test the mechanical stability of the electroless gold thin films. Various normal loads were applied to observe gold films resistance and to determine the films failures. At each load, seven tests were performed to provide a credible set of statistical data. 


\subsection{Data Analysis of 2D Gold Films}

The objectives of this study were to produce a reproducible and stable gold film with $50 \mathrm{~nm}$ thickness via electroless plating by utilizing self-assembled star polymers as templates, and to characterize the plated gold film by AFM, UV-visible

spectrophotometer, SPR, and RBS. The stability of the gold film was analyzed from the AFM images. Any morphology changes due to air exposures such as blistering or dewetting (if any) can be observed from the AFM images. From the AFM analysis, surface roughness of the plated films was determined by the RMS (rough mean square) analysis that is provided by the AFM software. The RMS determination was carried out in three different spots and an average value was taken.

The gold film thickness was analyzed by the RBS spectroscopy. Measurements were conducted in five spots over a single quartz or SF11 substrate. The multiple spots measurements were also useful in determining film uniformity. In the plating time versus film thickness study, the average thickness from five different measurements along oneinch wafer was used. Reproducibility was examined from batch-to-batch basis and dayto-day basis. In this case, reproducibility refers to film thickness and SPR response. 


\section{CHAPTER FIVE}

\section{RESULTS AND DISCUSSION}

This chapter summarizes the results obtained in the formation of $2 \mathrm{D}$ and $3 \mathrm{D}$ star polymer-templated gold nanostructures. The discussion for the 2D star polymertemplated gold films is divided into sections based on the sequential steps used in the research approach depicted in Figure 28. It starts with the deposition of the star polymers as an adhesive layer, followed by the gold seeds attachment and gold seed enlargement (growth). An additional section compares the star polymer with other adhesive templates. The discussion for the 3D gold nanoshells is focused upon the gold seed attachment and the growth process since 2D surface-confinement of star polymers is not required.

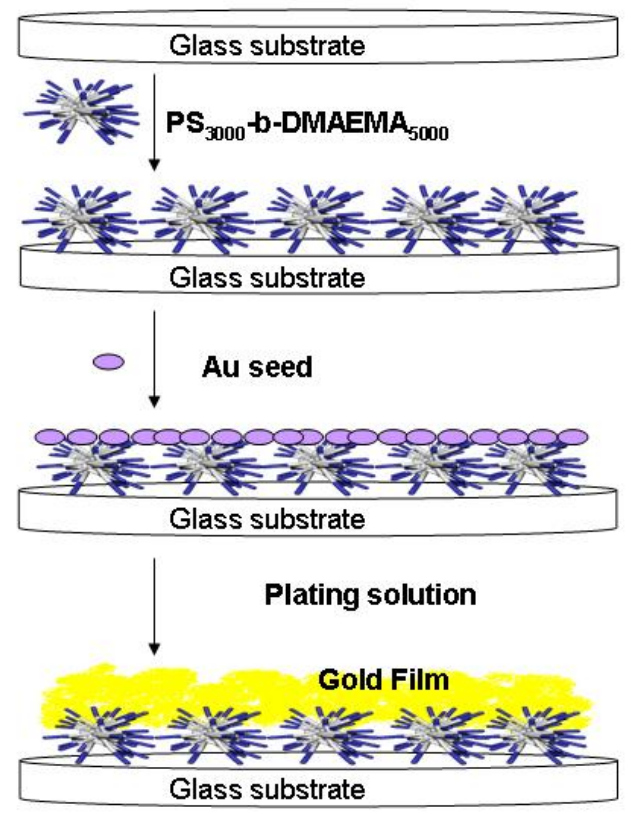

Figure 28. Schematic illustration of 2D electroless gold film formation by utilizing star polymers as templates. 


\subsection{Self-assembled Star Polymer PS-DMAEMA as Adhesive Template}

In general, amine groups interact with silanols group via electrostatic interaction that results in a amine-silanol complex [58]. Due to the availability of amine groups on the star polymer, it was postulated that star polymers formed amino-silanol complexes with the silanols on glass or silicon substrates leading to a strong attachment of the star polymers onto glass or silicon wafers. This postulate was confirmed through AFM images of the star polymer layer. The left image in Figure 29 shows the morphology of a silicon wafer that was treated with UV/Ozonolysis followed by a Millipore rinsing. A granular morphology was observed upon exposure of this silicon wafer to the star polymer solution, as shown in the right image of Figure 29. The grain size ranged from 20-25 $\mathrm{nm}$. The size range is in agreement with the light scattering data done in solution (21 $\mathrm{nm}$ in diameter).
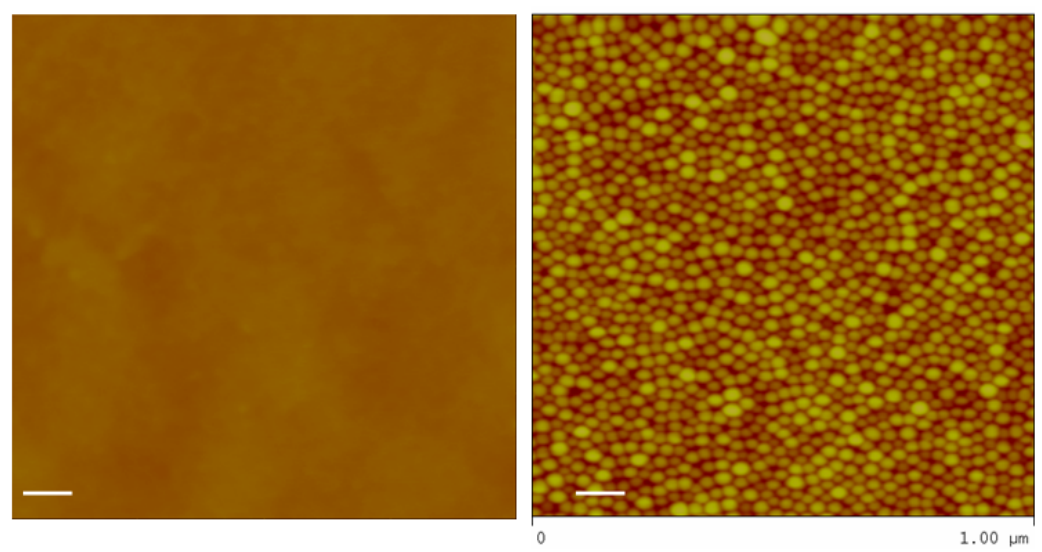

Figure 29. AFM images of a UV/ozone-treated silicon wafer: before (left) and after the deposition of star polymer PS-DMAEMA (right). Scale bar: $100 \mathrm{~nm}$; Z height: $30 \mathrm{~nm}$.

The star polymer also formed a stable monolayer and displayed a closely packed coverage over a $1 \mu \mathrm{m} \times 1 \mu \mathrm{m}$ area despite the substrates which the star polymers were 
being deposited onto, as shown in Figure 30. In this case, glass quartz and silicon wafer were used in comparison and the dipping technique was used to deposit the star polymers. The different substrates showed no obvious differences in star polymer size, shape, and distribution. From AFM images in Figure 29 and Figure 30, it can be also concluded that the dipping technique was effective in forming a monolayer without introducing any significant contaminants.
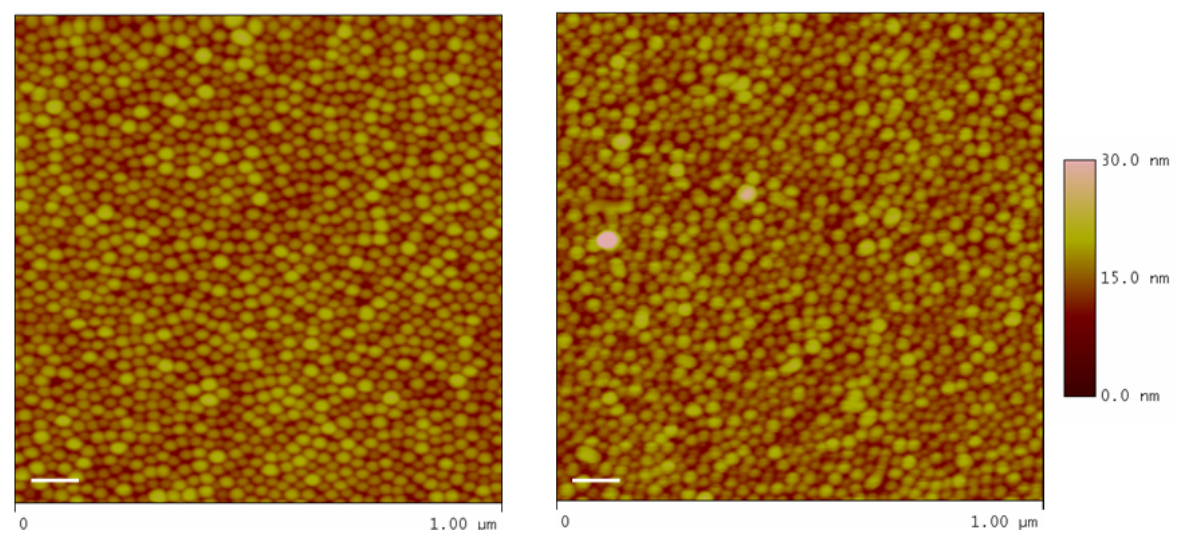

Figure 30. AFM images of star polymer PS-DMAEMA (water base) that was deposited on a silicon wafer (left) and a quartz wafer (right) by dipping. Scale bar: $100 \mathrm{~nm}$.

Following the successful deposition of star polymer as a monolayer, the next step was to optimize the dipping time. Previous studies showed that an adhesive layer, such as APTMS and PDDA, required hours for optimal deposition time [24, 25]. Thus, a rapid formation of star polymer as a stable adhesive layer offers advantages, templating time in particular, over APTMS and PDDA. Three variations (5, 2, and 0.5 hours) in dipping time were used for this set of experiments. AFM results in Figure 31 revealed that there was no significant alteration in the star polymer morphology regardless of changes in dipping time. 

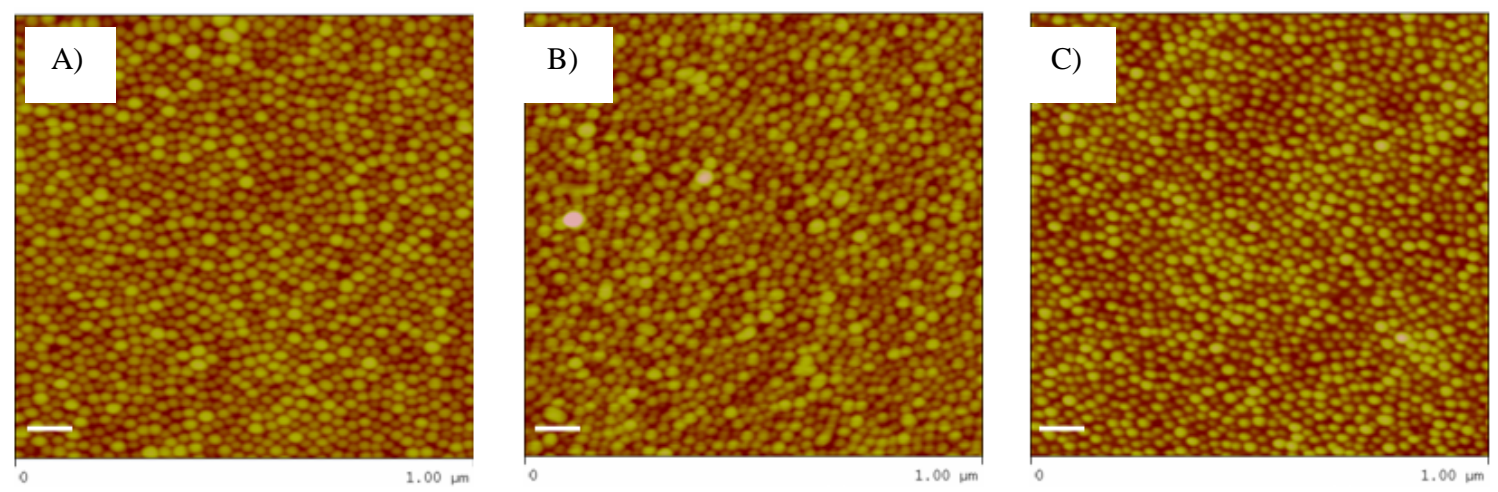

Figure 31. AFM images of star polymer PS-DMAEMA (water base) films that were formed in: A) 5 hour, B) 2 hour, and C) 0.5 hour dipping time. Scale bar: $100 \mathrm{~nm}$; Z height: $30 \mathrm{~nm}$.

In order to further optimize the dipping time, in situ observation with SPR spectroscopy was carried out. With the in situ experiments, the actual time required to form a stable star polymer layer was determined. After a water base measurement of SPR substrate was taken, the star polymer solution was flowed to the flow cell to interact with the SPR substrate. The deposition was then monitored with the kinetics and scan mode of the SPR spectroscopy. Figure 32 shows how significantly fast the star polymer deposition was. It took only seconds to achieve a stable jump in the kinetics spectra which reflected the rapidity of the star polymer deposition. An obvious resonance angle shift was observed even after triple rinsing by injecting water to the flow cell, as shown in Figure 32 (scan mode). In general, some materials show an increase in the signal intensity of the kinetics mode but when rinsing is applied, the materials get washed off due to weak interactions between the materials and the SPR substrates. As a result, there is no resonance angle shift in the scan mode. In this case the observable resonance angle 
shift in the star polymer deposition can be translated into a sufficiently strong attachment of the star polymer onto the SPR substrate via electrostatic interactions.
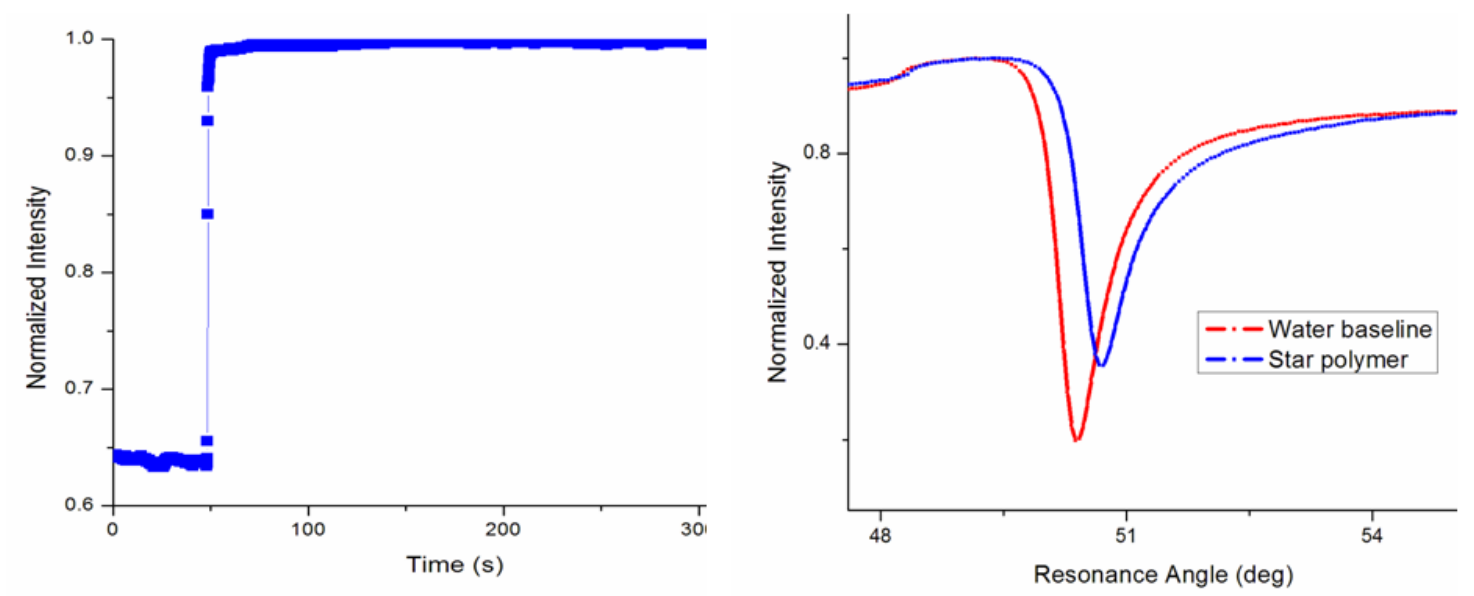

Figure 32. The rapid formation of star polymer PS-DMAEMA (water base) layer as traced by SPR spectroscopy (left: kinetics mode; right: scan mode).

Reviewing the AFM and SPR results, it was concluded that the star polymer was soluble in water and could be deposited as a monolayer on either glass or silicon dioxide substrate. The deposition occurred in seconds and the resulting films were stable in dry state. The rapid deposition of star polymer can be attributed to the large attachment sites provided by multiple amines in the periphery. Electrostatic interactions between these amines and silanols on the glass or silicon dioxide surface were also shown to be effective for anchoring the star polymer film. The synthesis of star polymer PSDMAEMA was shown to be robust. Figure 33 shows star polymer films that were originated from two different batches. The star polymers average diameter was shown to be consistent in the range of $20-25 \mathrm{~nm}$. 

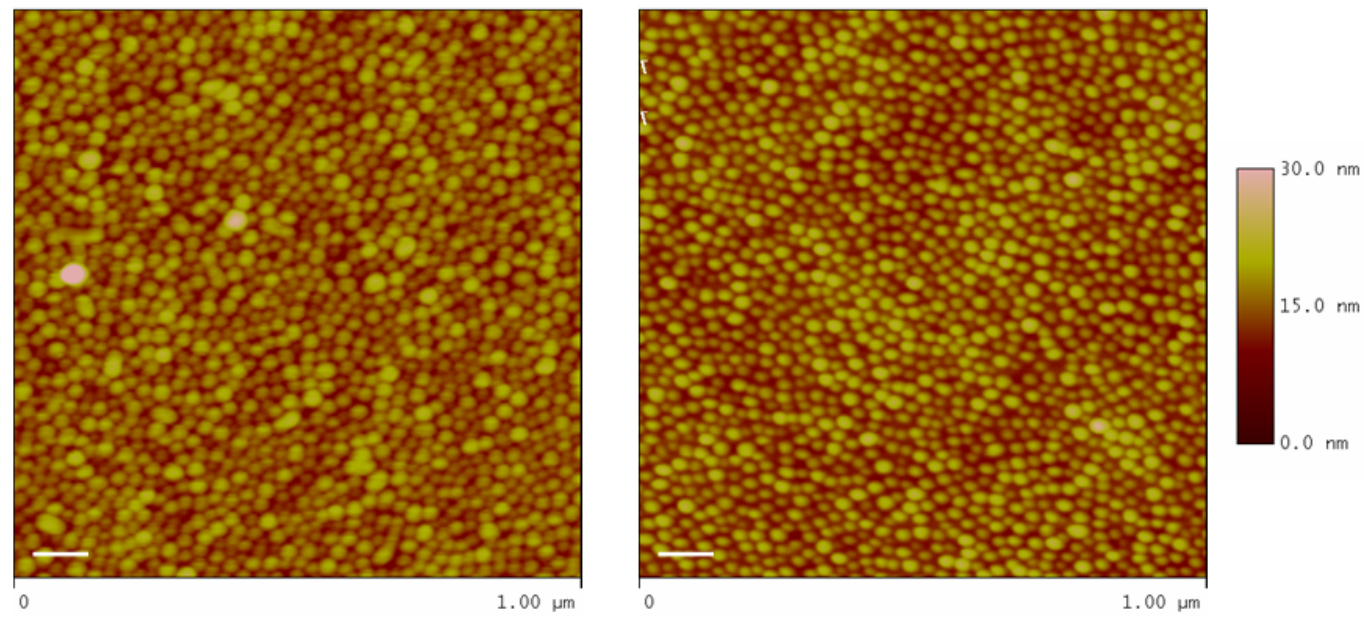

Figure 33. AFM images of star polymer PS-DMAEMA (water base) films that were produced from two different synthesis batches. Scale bar: $100 \mathrm{~nm}$.

\subsection{Gold Colloid Immobilization as Seeding Step}

\subsubsection{Gold Colloid Synthesis}

Many methods to produce gold colloids are available. Numerous combinations of different stabilizers and reducing agents can be used depending on the preferred final states of the colloids (size, shape, solubility, and etc.). In this research study, sodium citrate and sodium borohydride were chosen to produce water-soluble spherical gold colloids with an average of 3-5 $\mathrm{nm}$ in diameter. As soon as sodium borohydride was added to the mixture of gold (III) chloride and sodium citrate, the color of the mixture turned to a clear pink/purple color. The color change indicated the formation of the gold colloids, as suggested by Lei et al. [24].

Based on literature, spherical gold colloids with an average of $3 \mathrm{~nm}$ diameter display an absorbance in the visible region at around 506-508 $\mathrm{nm}[24,41]$. Therefore a UV-visible analysis was conducted to verify the gold colloid formation. The synthesized gold colloid solution was shown to exhibit an absorption peak at $510 \mathrm{~nm}$, as shown in 
Figure 34. The peak was shifted by $2 \mathrm{~nm}$ from the reference value $(508 \mathrm{~nm})$. This suggested that the size of the particles was larger than $3 \mathrm{~nm}$. To verify the particle size, the solution was then cast-dropped on a copper grid and sent for TEM analysis. The TEM image in Figure 35 shows that there is a wide particle size distribution ranging from 3-10 $\mathrm{nm}$ with an average of $5 \mathrm{~nm}$.

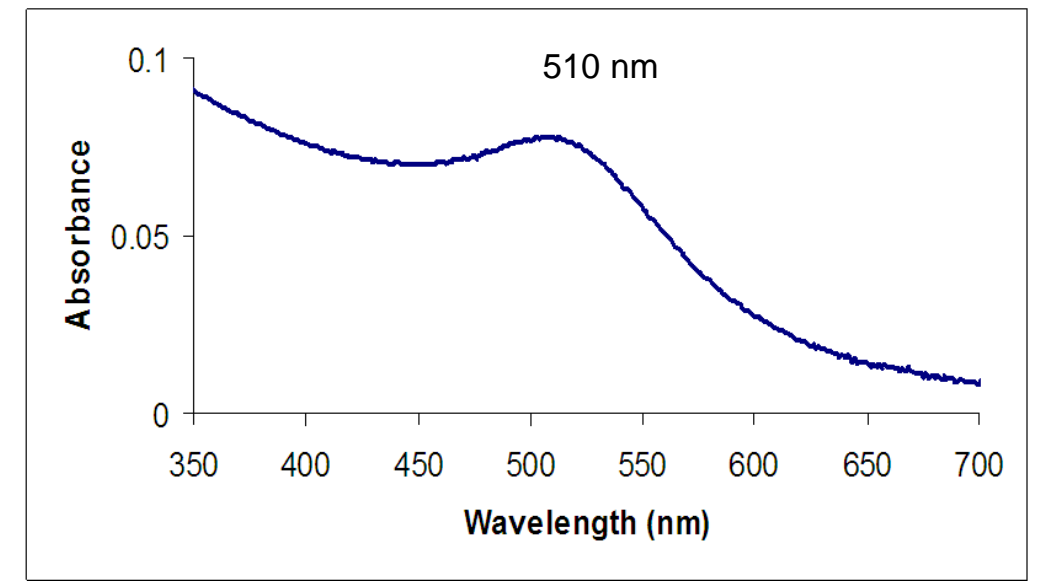

Figure 34. UV-visible spectra of a citrate-capped gold colloid solution.

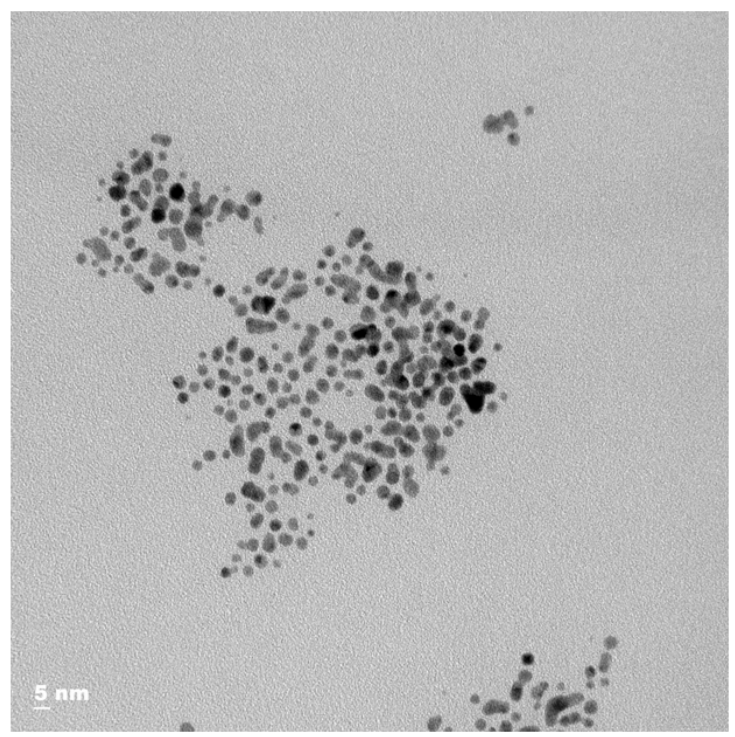

Figure 35. TEM image of citrate-capped gold colloids with an absorption peak at $510 \mathrm{~nm}$ as shown in Figure 34. 
Since the size of the particles was larger than expected, a $\mathrm{NaBH}_{4}$ calibration was conducted. It was possible that the crude $\mathrm{NaBH}_{4}$ used had partially decomposed, so its efficacy in reducing the gold ions was diminished. The blue spectrum in Figure 36 refers to the gold colloids with $0.2 \mathrm{~mL} \mathrm{NaBH}_{4}$ addition (the original volume that was used to produce gold particles in Figure 24) while the red, yellow, and green spectra refer to the gold particles with $0.25,0.30$, and $0.40 \mathrm{~mL} \mathrm{NaBH}_{4}$ addition, respectively. As the amount of $\mathrm{NaBH}_{4}$ was increased, the absorbance peak blue-shifted towards a shorter wavelength. This indicated that a reduction in the size of the particles might have occurred. It also demonstrated that the $\mathrm{NaBH}_{4}$ had decomposed since an additional volume of $\mathrm{NaBH}_{4}$ was required to achieve the preferred gold particle size. It is worth noting, that over a period of storage time, a calibration of $\mathrm{NaBH}_{4}$ might be required.

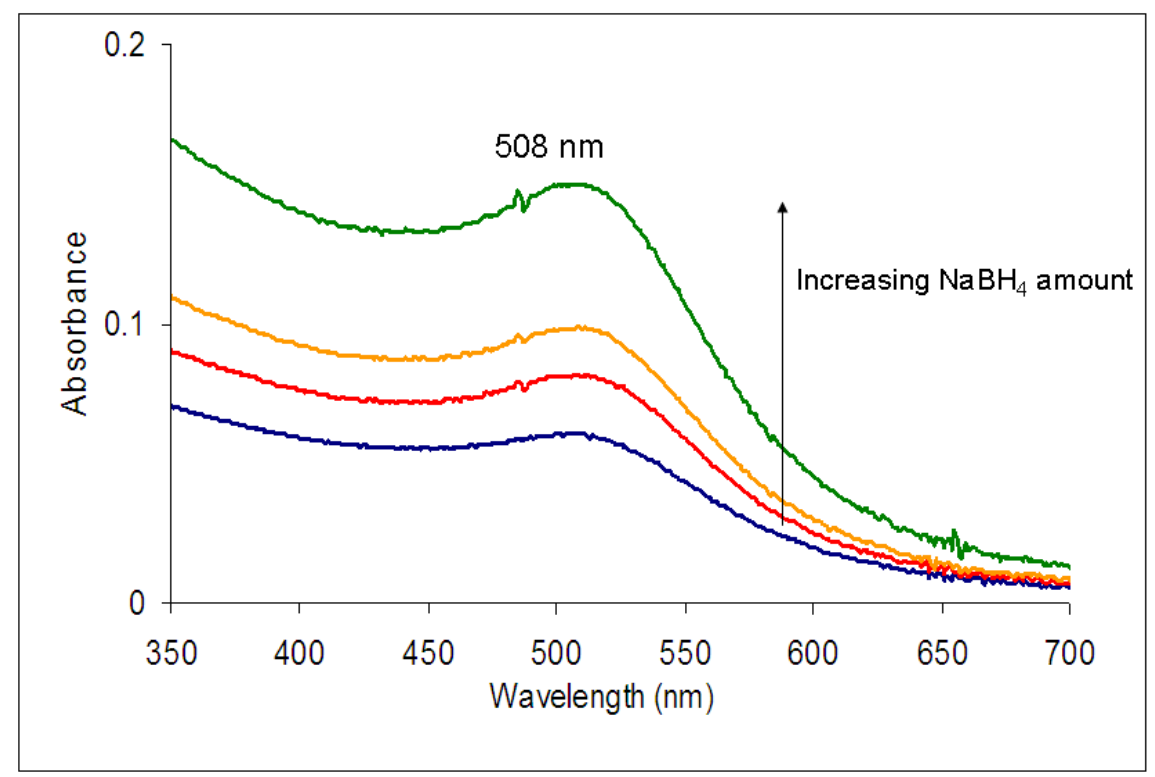

Figure 36. Red-shifted UV-visible spectra of gold seed solution due to an increase in $\mathrm{NaBH}_{4}$ volume. 
TEM analysis was further performed to verify the size of the particles.

Interestingly, the diameter of the particles was still in the $5 \mathrm{~nm}$ range. However, an improvement in the particle size distribution was observed, as shown in Figure 37. Hrapovic et al. [25] suggest that the quality of electroless gold film deposition depends more heavily on the seed size distribution rather than the seed size. Thus, it was decided that the procedure to produce a narrow distribution of $5 \mathrm{~nm}$ spherical gold particles was maintained throughout this research.

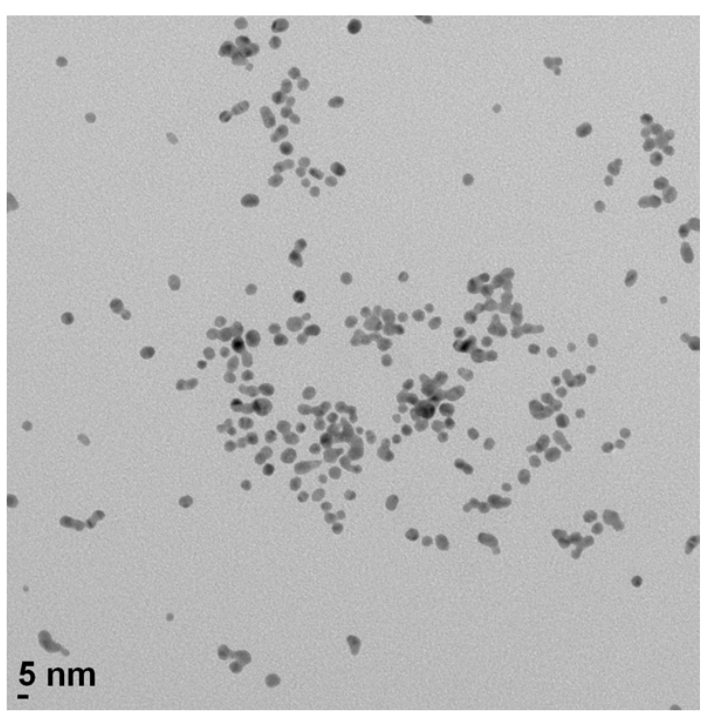

Figure 37. A narrower size distribution of the gold seeds was achieved by doubling the $\mathrm{NaBH}_{4}$ volume (UV-visible spectrum of this solution is displayed in Figure 41: the green spectrum).

The repeatability and reproducibility of the gold seed synthesis were also demonstrated in Figure 38 and Figure 39. Figure 38 shows the same absorbance peaks of two gold seed solutions made in the same day from the same gold chloride, sodium citrate, and sodium borohydride stock solution. 


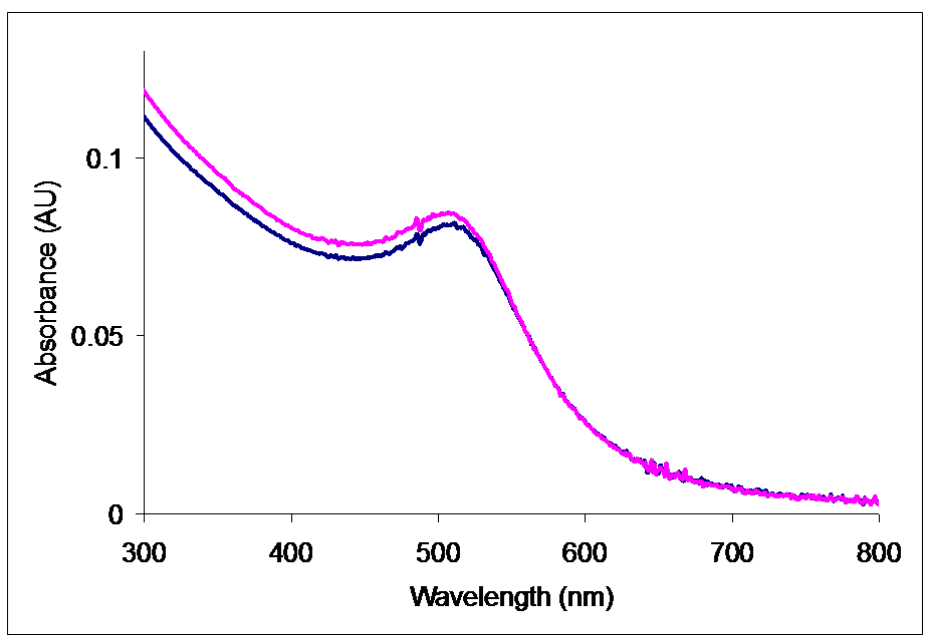

Figure 38. The repeatability of citrate-capped gold colloid synthesis as analyzed by UVvisible spectrophotometer.

Figure 39 shows the same absorbance peaks of gold seed solutions that were made individually in a five months period. Each solution was made from different gold (III) chloride, sodium citrate, and sodium borohydride solutions on different days. It is very important that the gold seeds had the same state in every experiment. The same conditions at the seeding step eliminated a key variable in the growing/plating step study.

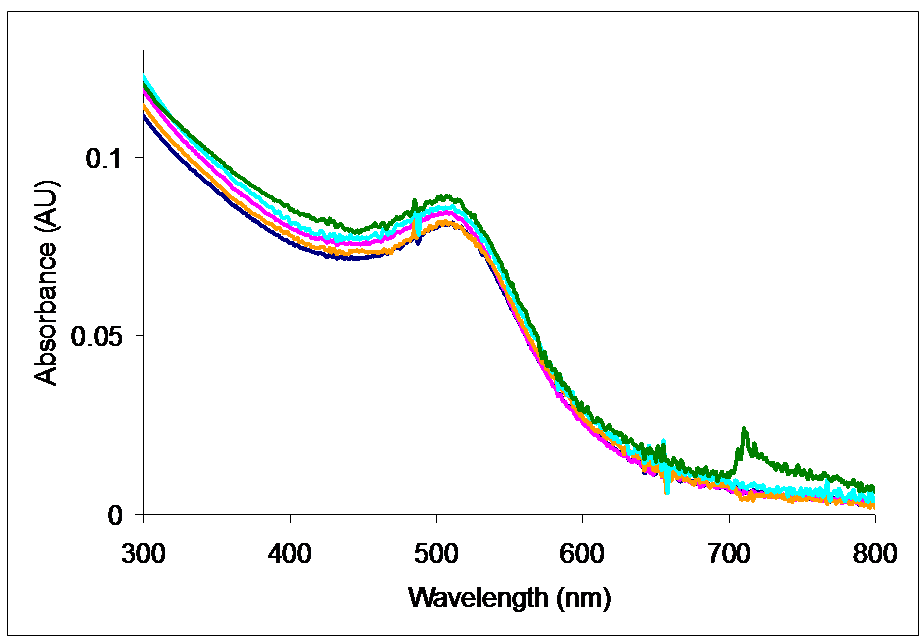

Figure 39. The reproducibility of citrate-capped gold colloid synthesis as traced by UVvisible spectrophotometer. 


\subsubsection{Gold Seed Attachment to Star Polymer Film}

The most significant aspect of the seeding step is to make sure that the star polymer can interact with the gold seeds and immobilize them in a dense layer form. Without a proper seed layer formed on top of the star polymer layer, there will be no gold growth/plating. The real-time SPR spectroscopy was chosen to monitor the feasibility of this gold seed attachment to the star polymer layer. The seed solution was flowed into the flow cell to interact with a star polymer-templated SPR substrate. This star polymer was deposited in situ onto the SPR substrate prior to the seeding step. From the kinetics plot shown in Figure 40 (left), a significant jump in the intensity indicated that there was a rapid deposition of gold seeds onto the star polymer layer. After a triple rinsing with Millipore water was performed, a regular scan was performed. The resonance angle was shifted indicating that the gold seeds interacted firmly with the star polymers. After the SPR substrate was taken out from the flow cell setup, it was clearly seen that the area that was exposed to the seed solution exhibited a purple color.
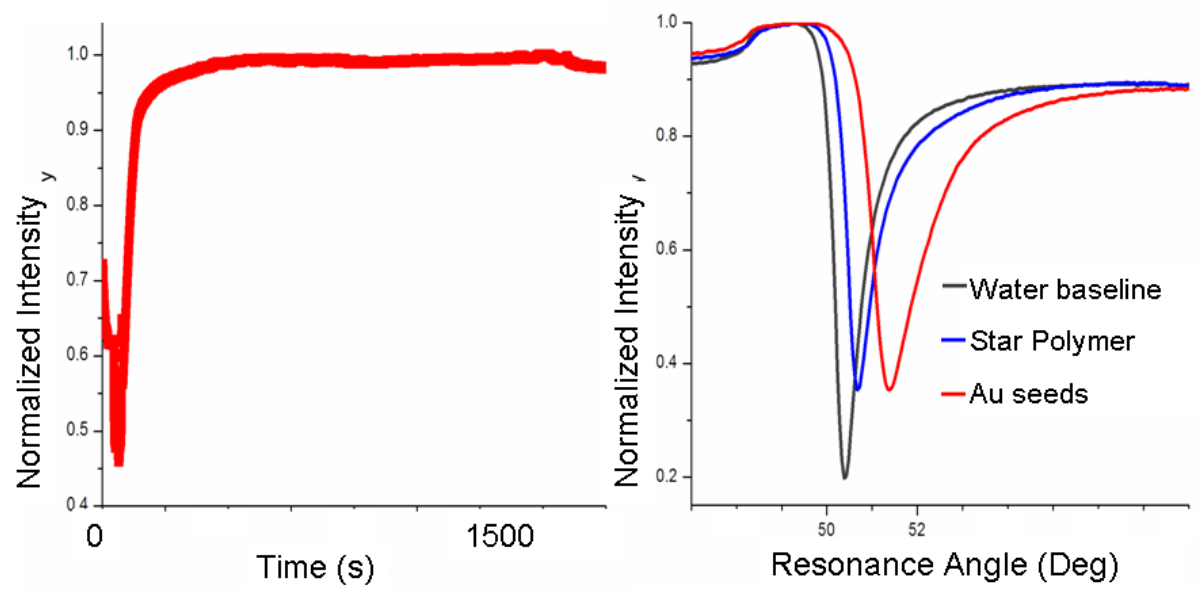

Figure 40. SPR curves of citrate-capped gold colloid deposition on a star polymertemplated glass surface (left: kinetics mode; right: scan mode). 
The seed attachment experiment was repeated. Instead of in situ deposition, deposition by dip coating was performed. The SPR substrate was also changed to a blank quartz wafer so that any changes were traceable by UV-visible spectrophotometer. The red spectrum in Figure 41 is the spectrum of star polymer-templated quartz that was exposed to gold seed solution for one hour. Compared to the peak of gold seeds in solution (the blue spectra in Figure 41), a red shift was observed. This phenomenon also occurred in the study conducted by Grabar et al. [59]. The $13 \mathrm{~nm}$ gold particles in their study projected a peak at $520 \mathrm{~nm}$ when in solution. When the particles were immobilized on an organosilicate layer, the peak shifted to a longer wavelength.

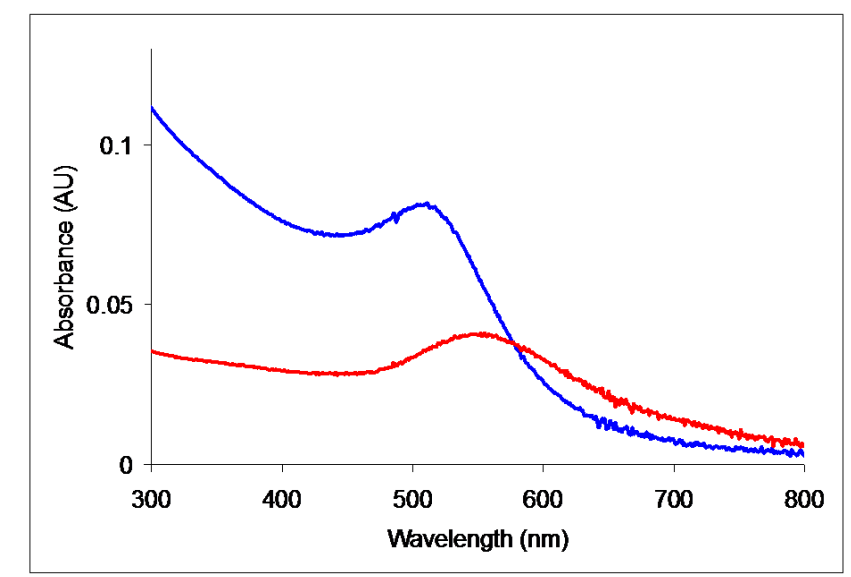

Figure 41. UV-visible spectra of citrate-capped gold colloids: in solution (blue) and after being immobilized on a star polymer-templated glass surface (red).

The effect of this gold seed immobilization on the star polymer layer was then examined using AFM, and the AFM images are presented in Figure 42. The star polymer film appeared intact without any dewetting problems although an inflated condition seemed to appear after the deposition of the gold seeds. Since the gold seeds are spherical and in the range of $5 \mathrm{~nm}$ while the star polymers themselves form spherical 
shapes, AFM tip convolution may have contributed to the fact that individual seeds were hard to see.
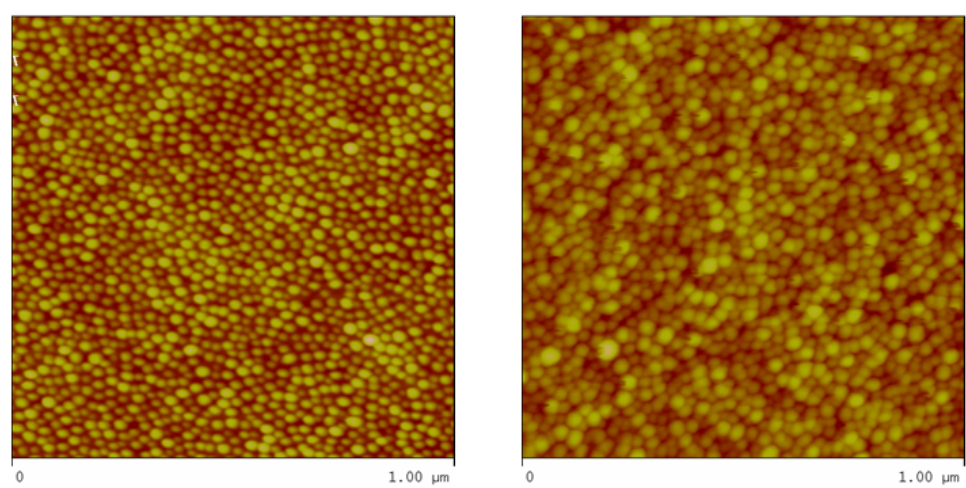

Figure 42. AFM images of a star polymer film on a glass surface before (left) and after (right) a seeding process ( $\mathrm{Z}$ height: $30 \mathrm{~nm}$ ).

The optimum seeding time was also studied. Previous studies have shown that at least 12 hours were required to develop a proper seed layer [24, 25]. However, referring to the kinetics plot of the SPR data derived from the deposition of gold seeds on star polymer layer (Figure 40), it can be seen that the deposition of gold seeds happened in less than one hour. Thus, the variation for seeding time in this study was ranged from 5 to 60 minutes and the results are shown in Figure 43. There was no significant increase in the absorbance for the 5 and 15 minute seeding times. However, a considerable increase in the absorbance from 15 to 30 minutes was observed. As for the 30 and 60 minute seeding times, there was only a slight increase in absorbance. It can be concluded that 30 minutes is sufficient for the seeding time although 60 minutes can be applied to ensure a complete deposition. Later in the next section of this chapter it will be demonstrated that $5,15,30$, and 60 minute seeding times generated gold films that were 
similar in terms of thickness and uniformity (at constant growth time and growth solution concentration).

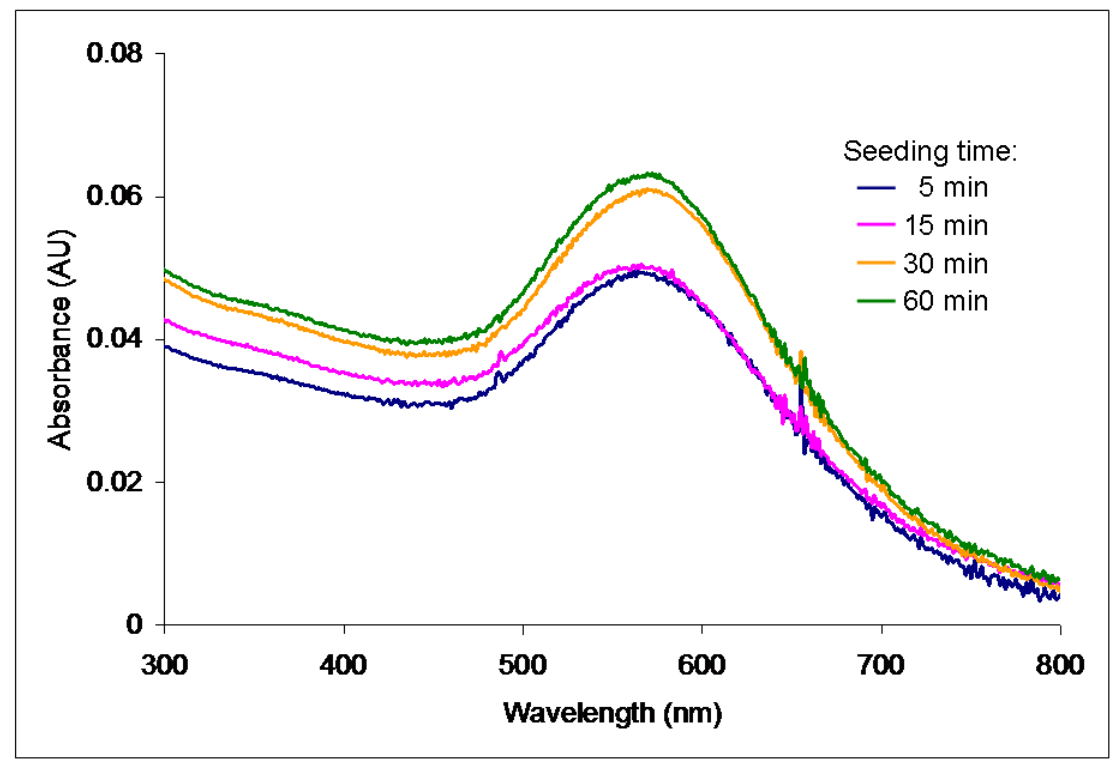

Figure 43. The resulting different absorption levels due to a variation in seeding time.

\subsection{Growth/Plating Process to Form Contiguous Gold Films}

\subsubsection{Effects of Growth Solution Concentration}

After the feasibility of gold seed deposition onto star polymer template had been established, the next step was to set up proper growth conditions to grow thin gold films on the star polymer-seeded films. As mentioned in the literature review, the most suitable growth solution is a mixture of gold (III) chloride and hydroxylamine hydrochloride. Thus, freshly made mixtures of $0.1 \% \mathrm{w} / \mathrm{v}$ gold (III) chloride and hydroxylamine hydrochloride were used for the initial experiments. This specific concentration was used since it was effective in producing gold films in the study carried out by Lei et al. [24]. In situ monitoring by SPR was selected as the first characterization 
method. From Figure 44, the shift in the resonance angle after 50 minute growth step (red curve) can be translated into a successful deposition process.

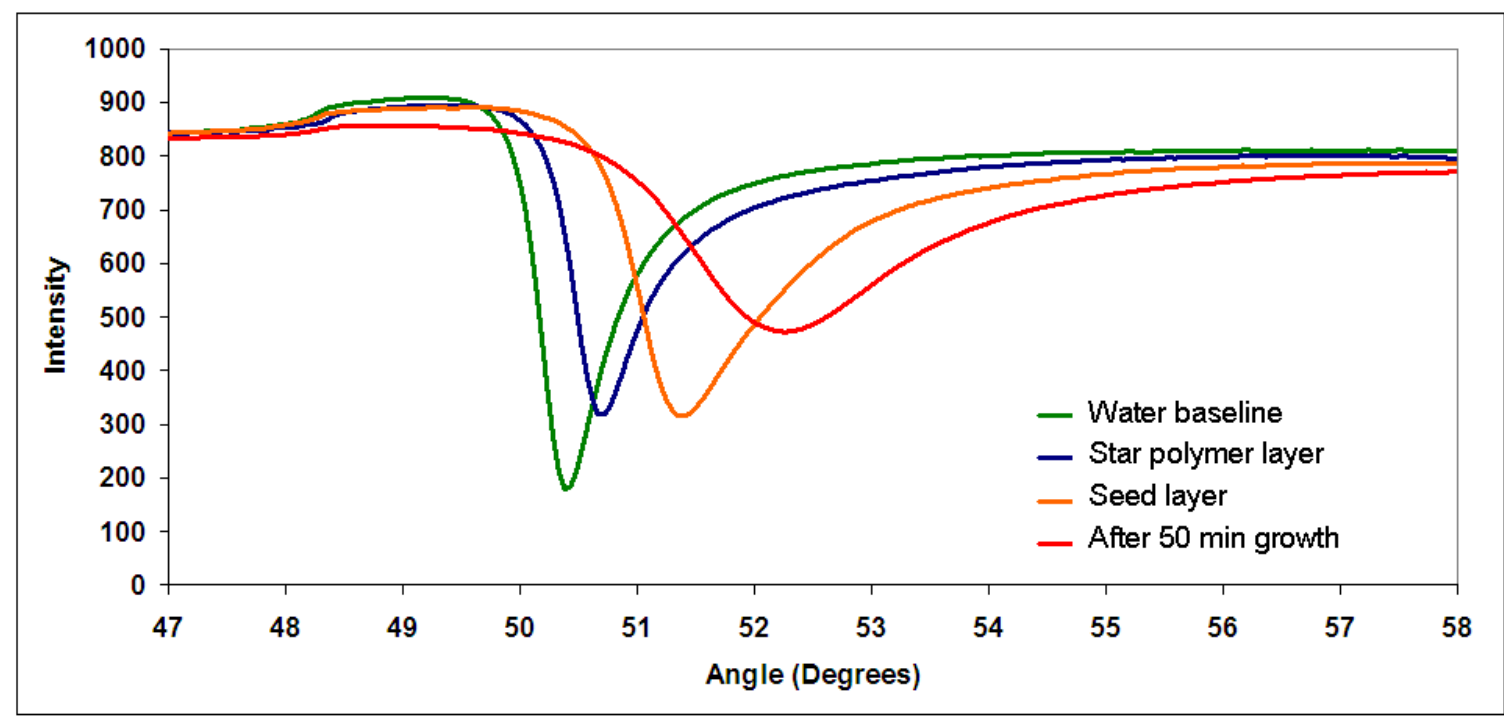

Figure 44. Growth process of the seeded star polymer film as monitored in situ by SPR spectroscopy.

AFM analysis was then carried out to confirm this deposition was due to the enlargement of the gold seed particles. Samples for the AFM analysis were generated from dipping process instead of the in situ SPR flow cell. The dipping process was chosen so that multiple samples could be grown from the same growth solution batch and any color changes on the substrate could be instantly observed. Based on previous studies, growth step can be determined by the color changes on the substrate from pink to blue to finally reflective gold luster $[24,41]$. From AFM images shown in Figure 45, it is apparent that the gold particles grew. It was also noticeable that the longer the plating time, the larger the particles that were generated. 

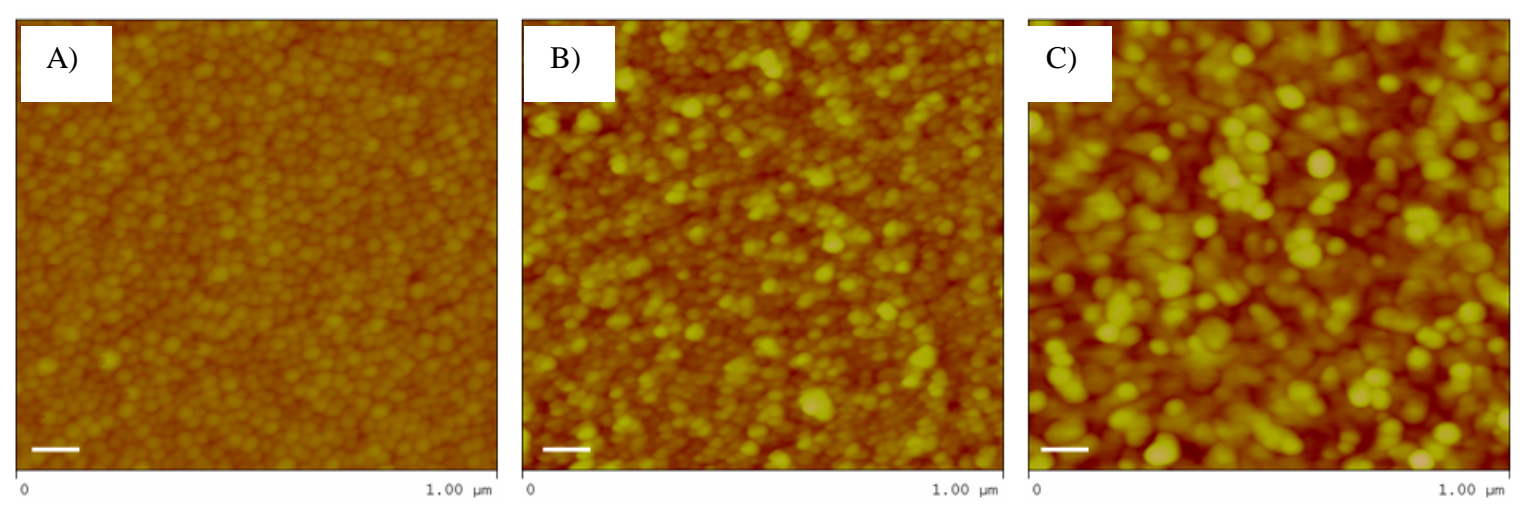

Figure 45. AFM images of gold seeded star polymer: A) before growth step, B) after 5 min growth, and C) after 10 min growth with a $0.1 \%$ growth solution. Scale bar: 100 $\mathrm{nm}$; Z height: $80 \mathrm{~nm}$.

One of the objectives in this work was to produce thin gold films that would generate good SPR signals. Therefore, the next experiment was designed to obtain SPR signals from the star polymer-templated gold films. The growth solution concentration was set at $0.1 \% \mathrm{w} / \mathrm{v}$ while the growth time was varied until a SPR signal was generated. It was found that a good SPR signal was generated from a gold film grown for 20 minutes as displayed in Figure 46B). After the 20 minutes of growth, reflective gold clusters were formed randomly on the substrate. In the micrometer range, however, the film looked completely covered by the enlarged gold particles as shown in Figure 46A). AFM analysis also showed that the film roughness value was fairly low (RMS: $6.7 \mathrm{~nm}$ ). This is comparable to the RMS value obtained by Huang et al. [26] in their study (RMS: $6.8 \mathrm{~nm}$ for a $57 \mathrm{~nm}$ thick gold film). The SPR signal of this star polymer-templated gold film was equivalent to that of evaporated gold film, as depicted in Figure 47. The difference in the resonance angle can be attributed to a slight difference in the thickness of each gold film. 

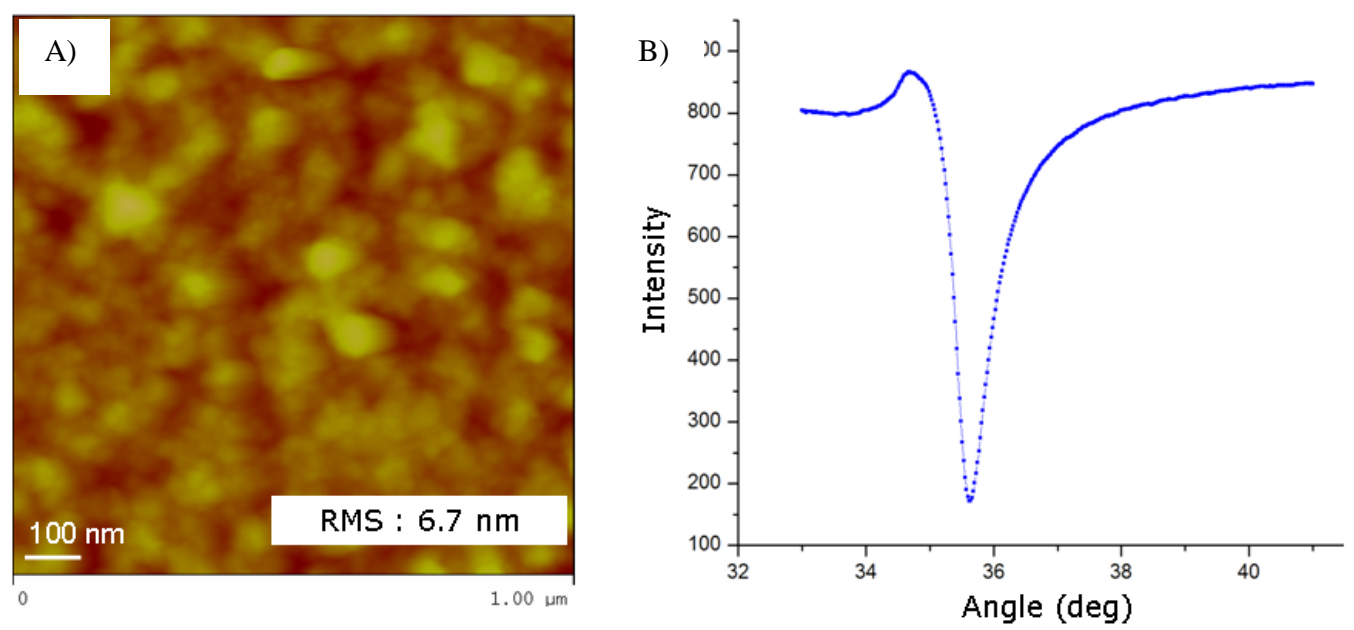

Figure 46. A) AFM image of a star polymer-templated gold film with a 20 min plating time and B) the corresponding SPR response.

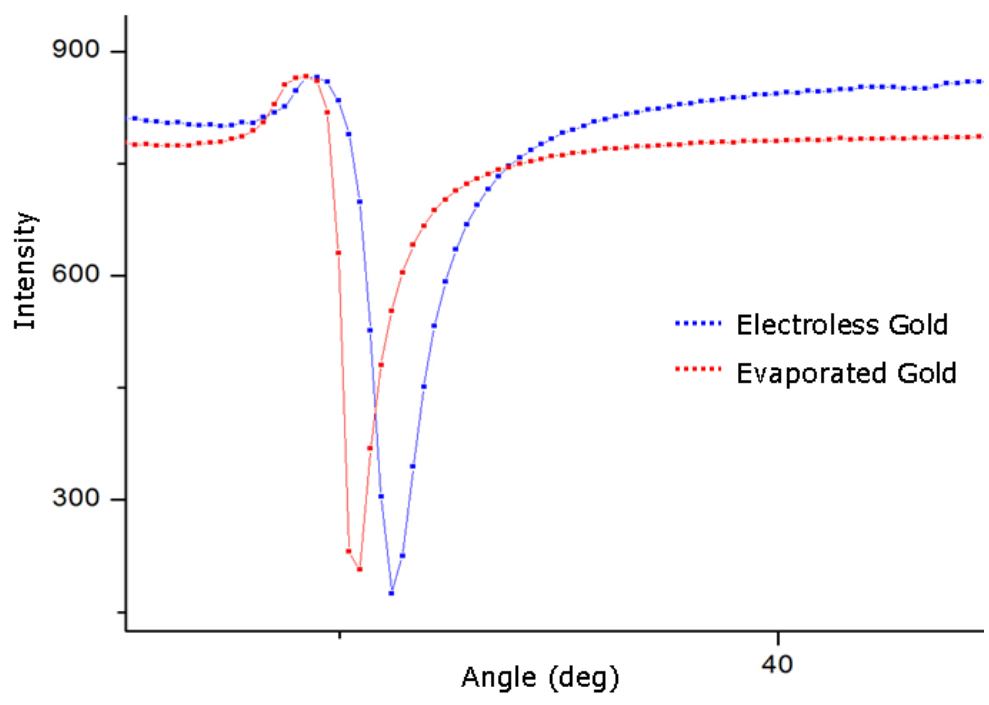

Figure 47. Comparison of SPR responses from an electroless plated gold film and an evaporated gold film.

Since the preliminary results of the star polymer-templated gold films were promising, the next step was to reproduce these results with an improvement in the gold film appearance (more uniform gold luster across the substrate). All parameters throughout the templating, seeding, and plating process were retained. However, after a 
number of experiments were performed, similar results could not be achieved. The resulting gold films were still patchy with no SPR signal generation. Thus, the growth solution concentration was increased to $0.5 \% \mathrm{w} / \mathrm{v}$ from $0.1 \% \mathrm{w} / \mathrm{v}$. Plating time was decreased accordingly to a range of 4-5 minutes. Surprisingly, this resulted in visually uniform gold luster films across the substrates. The color changes on the substrates during the growth step were also more noticeable (from pink to blue to a final gold luster). The next parameter to be varied was the plating time that correlated to film thickness. In order to get an excellent SPR signal, the optimum gold film thickness is around $50 \mathrm{~nm}$ [38]. From Table 3 it can be noticed that the film thickness increased as the growth time increased. Two minutes was found to be the optimum growth time to generate a $45 \mathrm{~nm}$ gold film. The star polymer-seeded layers are believed to be the contributing factor for the $15-20 \mathrm{~nm}$ thickness difference from the alpha step and RBS measurements.

Table 3. Increasing gold film thicknesses resulting from longer plating times.

\begin{tabular}{|l|l|l|}
\hline \multirow{2}{*}{$\begin{array}{l}\text { Time } \\
(\min )\end{array}$} & \multicolumn{2}{|l|}{ Thickness (nm) } \\
\cline { 2 - 3 } & Alpha step & RBS \\
\hline 1 & 40 & 26.4 \\
\hline 2 & 60 & 45.7 \\
\hline 3.5 & 85 & 64.7 \\
\hline 4.5 & 90 & 69.2 \\
\hline
\end{tabular}

Besides the color changes on the substrate that were visually observed, the formation of thin gold films was traced by the UV-visible spectrophotometer. The green curve in Figure 48 shows what the UV-visible spectrum of a visually gold luster film 
(from electroless deposition) looks like. The absorption peak of the plated gold film (green spectrum) is blue-shifted to a longer wavelength compared to that of seeded film (red spectrum). This shift indicates that larger particles were formed. A similar absorption feature of plated gold film was also shown by the evaporated gold film (spectrum is not shown).

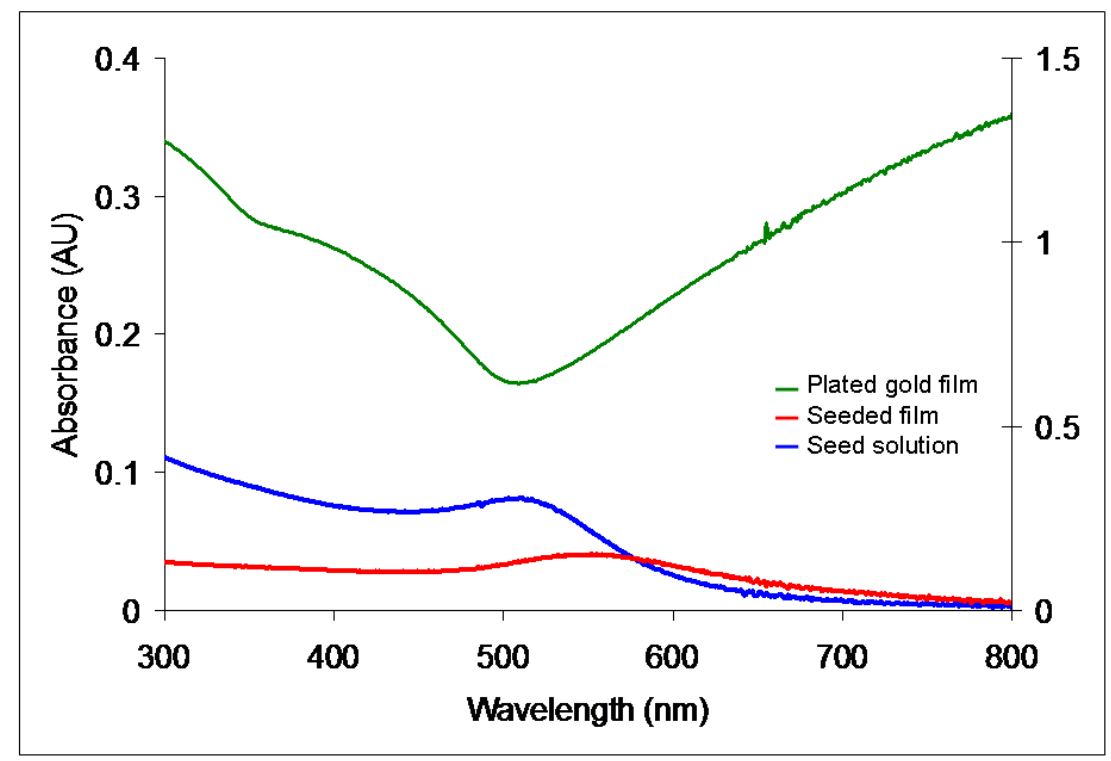

Figure 48. Seeding and growth steps in the electroless gold film formation as characterized by UV-visible spectrophotometer.

These UV-visible absorption spectra are similar to those obtained by Huang et al. [26] in their study. Their results are shown in Figure 49. Their seeded glass substrate showed an absorption peak at around $550 \mathrm{~nm}$. When their samples were exposed to a growth solution for different periods of time, blue-shifts to a longer wavelength occurred. 


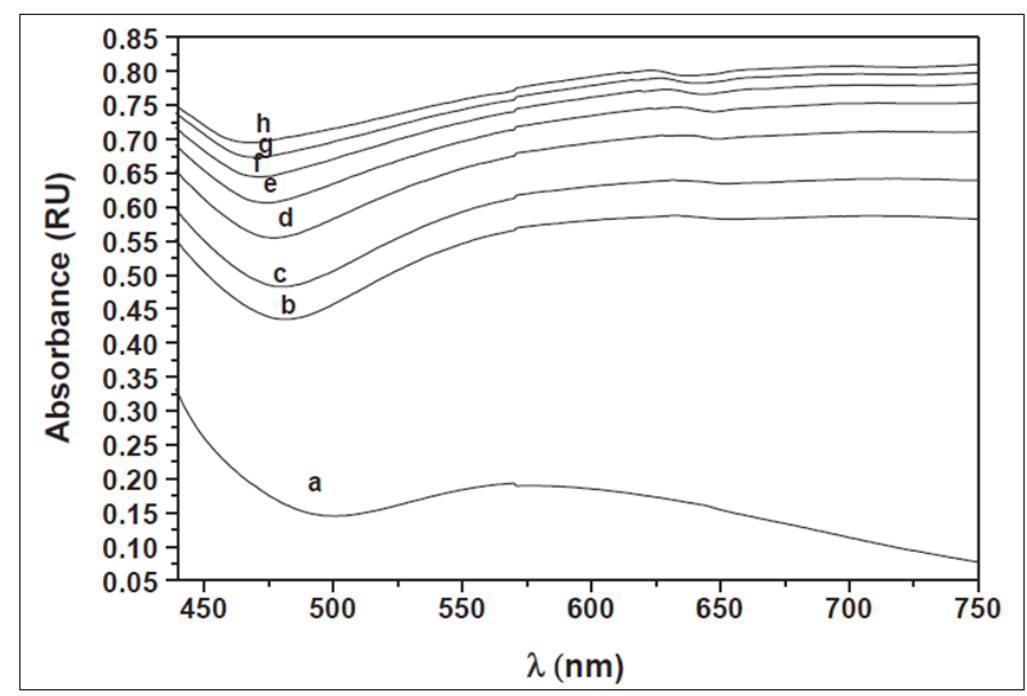

Figure 49. Absorption spectra of a gold-seeded layer (a) and plated gold films with various plating times (b-h) [26] (reprinted with permission from Elsevier).

The ease of monitoring the growth by UV-visible spectrophotometer can also be applied to qualitatively measure the film thickness. A higher intensity can be correlated to a thicker film. It can also be used to check for the reproducibility of the gold film formation, as shown in Figure 50.

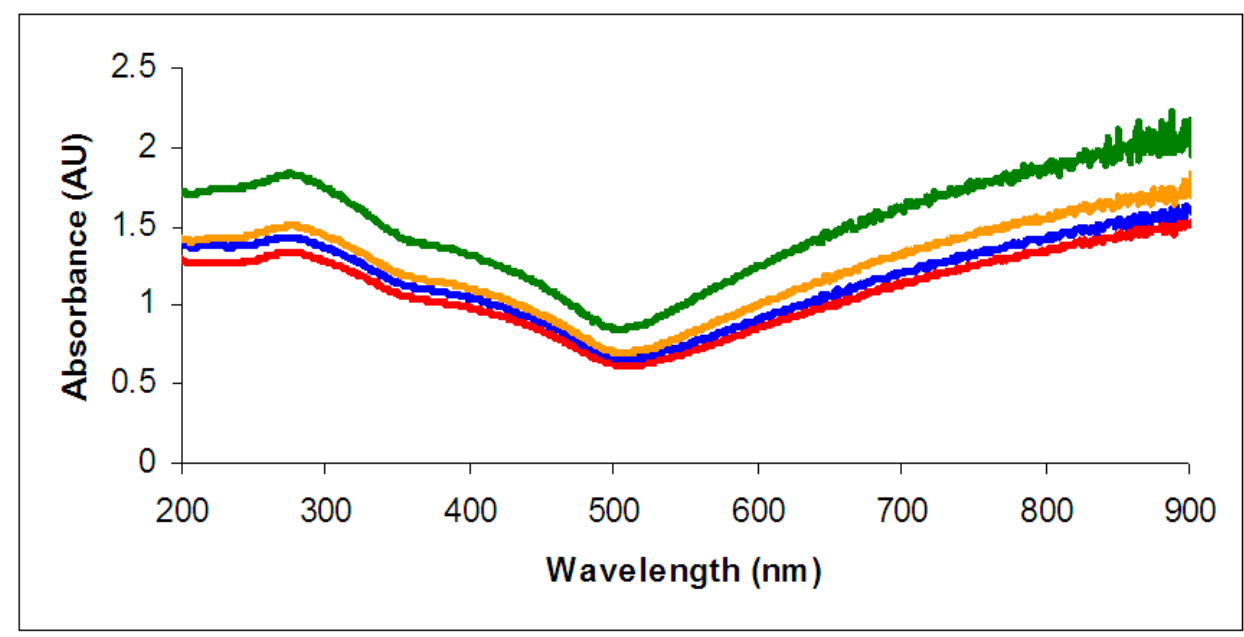

Figure 50. Qualitative measurement of gold film thickness carried out using UV-visible spectrophotometer. A higher absorbance is correlated to a thicker film. 
Although plating time plays a major role in determining the thickness of the plated gold films, it seems to have a lesser impact upon the average grain size and film roughness. Figure 51 shows the AFM images of four different gold films grown at different plating time, 1, 2, 3.5, and 4 minutes for A), B), C), and D), respectively. The thicknesses were in the range of 26.4-69.2 nm (each thickness can be found in Table 3). As the film became thicker, the grain size did not increase. It remained in the range of 50-60 nm although some clumps were observed in the thicker films (B), C), and D)). The film roughness (RMS value) was also found not to be a linear function of film thickness. These RMS values were lower than the RMS values reported by previous researchers [25, 26].
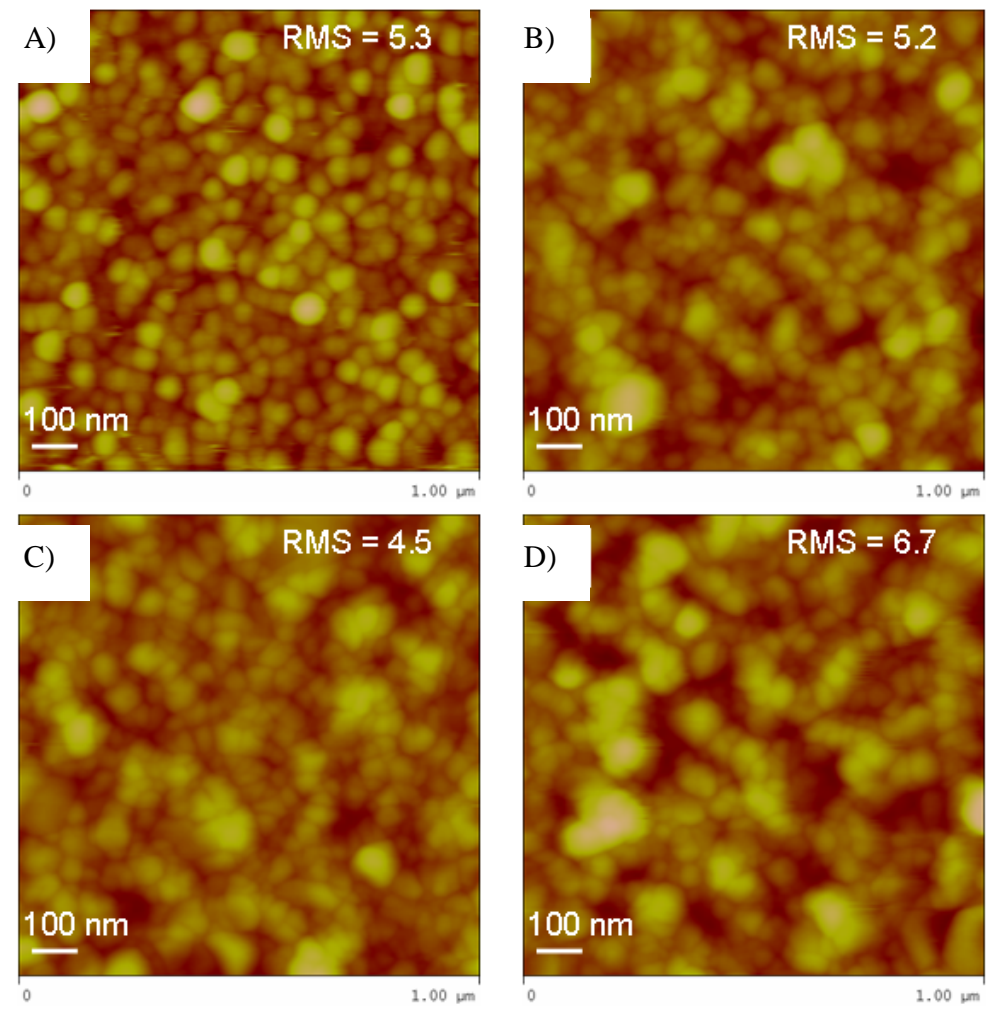

Figure 51. AFM images of star polymer-templated gold film that was plated for: A) 1 min, B) $2 \mathrm{~min}$, C) $3.5 \mathrm{~min}$, and D) $4.5 \mathrm{~min}$. The film thicknesses of A), B), C), and D) can be found in Table 2 . 
The use of $0.5 \% \mathrm{w} / \mathrm{v} \mathrm{HAuCl}_{4}$ and hydroxylamine hydrochloride as growth solution was found to significantly boost the reproducibility of the process, as shown in Figure 52. The data points are from three different batches and measured by alpha step and RBS. From this graph, a certain film thickness can be projected by adjusting the plating time accordingly. The difference in the measurement again confirms the presence of the star polymer-seeded layer underneath the gold film. In this case, alpha step measured the thickness of the overall film while RBS only measured the gold film.

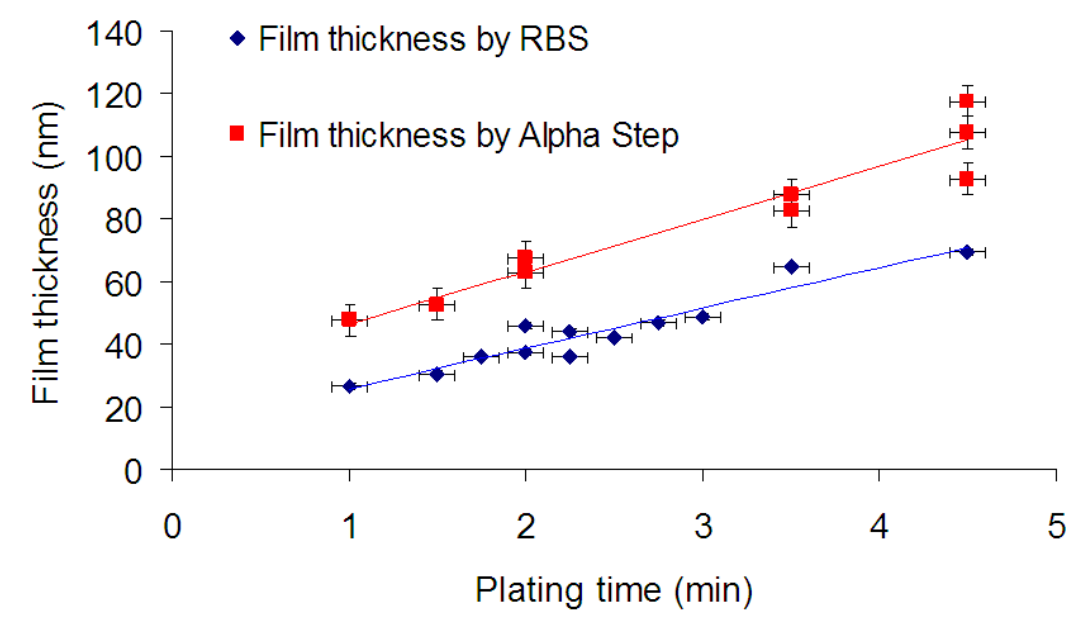

Figure 52. Film thickness reproducibility of the star polymer-templated gold films.

All processes in this project were done manually. A difference in seconds when a substrate was pulled out of the growth solution resulted in a significant film thickness difference. By lowering the growth solution concentration, the reaction rate is assumed to be suppressed. The working window can be stretched so that a slight difference in pulling the substrates out of growth solution will not significantly affect the films thicknesses. From Figure 53, it can be observed that film thickness is a linear function of growth solution concentration (at a constant plating time). Lower growth solution 
concentrations increase the precision of the plating process. However, since the $0.5 \%$ w/v $\mathrm{HAuCl}_{4}$ and hydroxylamine hydrochloride mixture was already producing reproducible gold films, this concentration was used for the latter experiments in this project.

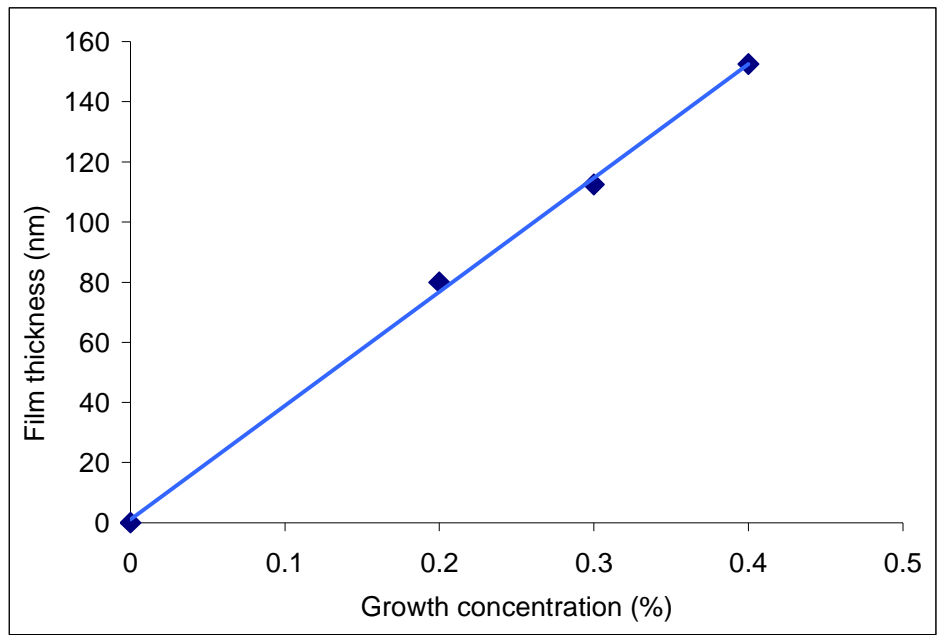

Figure 53. Film thickness as a linear function of growth solution concentration (at a constant plating time).

\subsubsection{Effects of Agitation on Film Uniformity}

The effect of agitation was also explored in this project. Previous studies mentioned the importance of agitation on film uniformity [24-26]. A circular shaker at $120 \mathrm{rpm}$, as suggested by Lei et al. [24], was initially used in this work. The $120 \mathrm{rpm}$, however, was found to be too fast for the experimental setup. Instead, 65 rpm was shown to be the optimum agitation rate in this study. When agitation was applied, the deposition rate increased. This resulted in thicker films at the same plating time, as shown in Figure 54. As the plating time gets longer, the deposition rate significantly increases. The agitation seems to remove the mass transfer limitation. Assuming the surface of star 
polymer-seeded layer is non-porous (thus removing diffusion limitation), the growth process depends solely on the reaction rate [73].

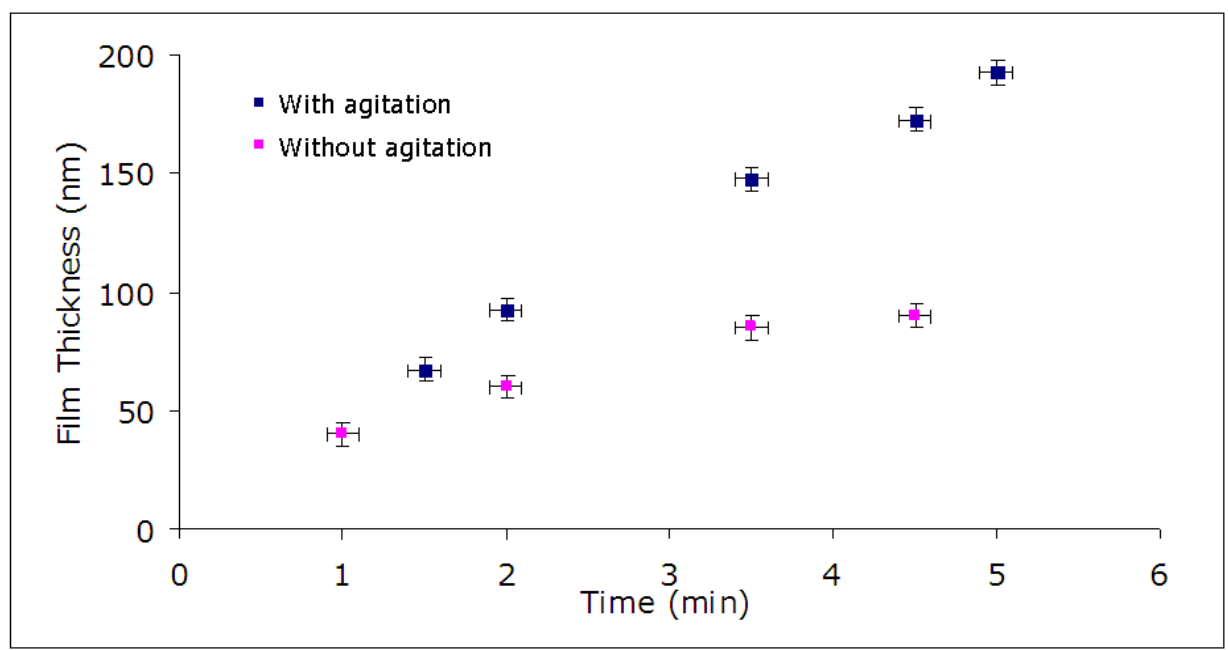

Figure 54. The effects of agitation on gold film thickness.

The next parameter analyzed was film uniformity across a one-inch wafer. RBS measurements for each film were done at five different points with one point in the center of the wafer and the other 4 points were $10 \mathrm{~mm}$ apart from the center. Four films from the same batch (with different plating times) were analyzed. The different plating times in this case correlated to different film thicknesses. The RBS results showed that those films were not uniform across the wafers. The edge region of each film was consistently thicker by $25 \%$ compared to the center part. The data is presented in Figure 55 . The agitation method was then changed to address this non-uniformity issue. In the previous setup, the seeded samples were exposed on the surface of the agitating growth solution. The new protocol was to let the seeded samples settle on the bottom of reactor, as shown in Figure 56. 


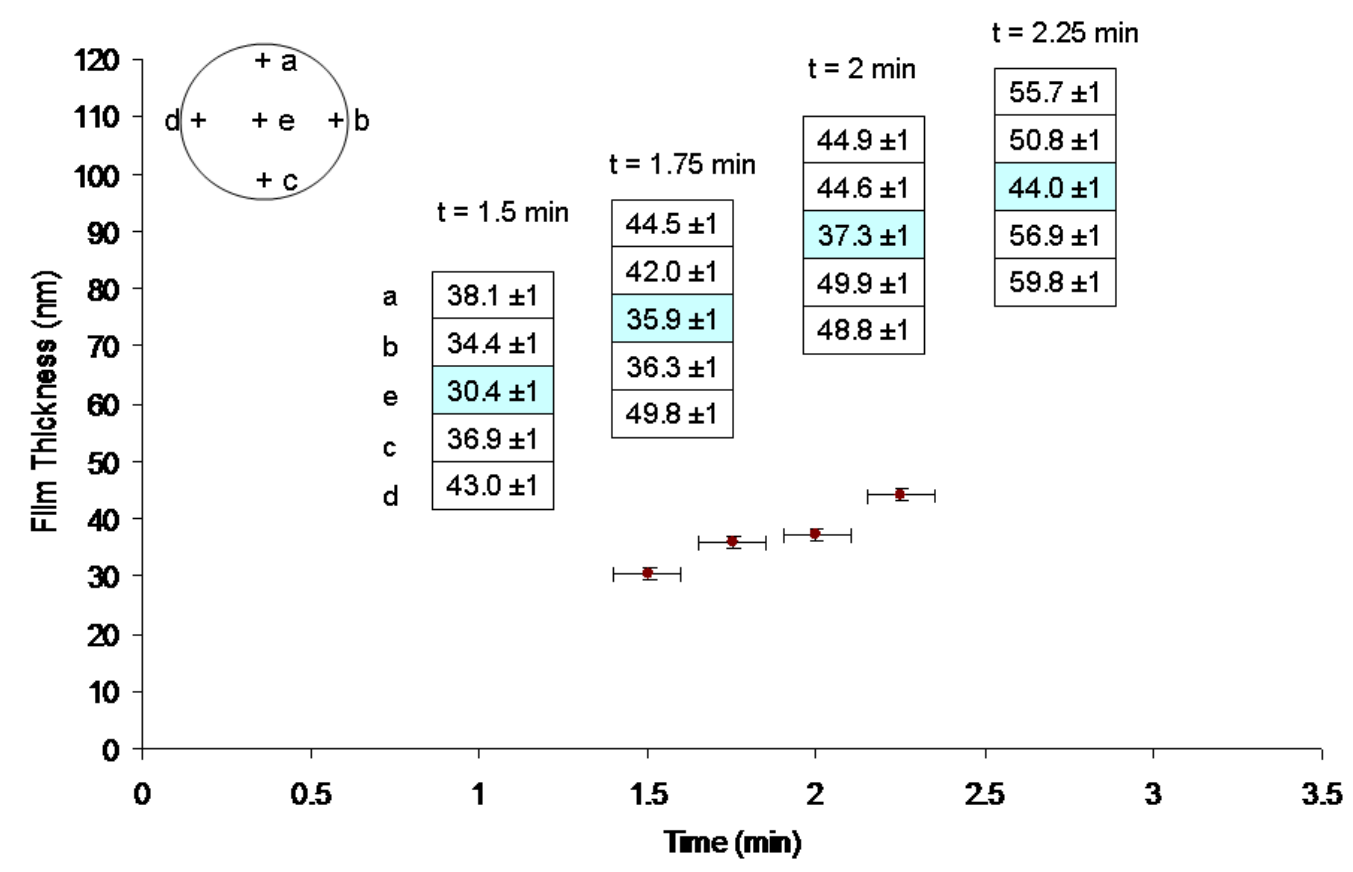

Figure 55. Thickness variances were observed along one-inch wafers with the center area having $25 \%$ less thickness compared to the edges.

A)

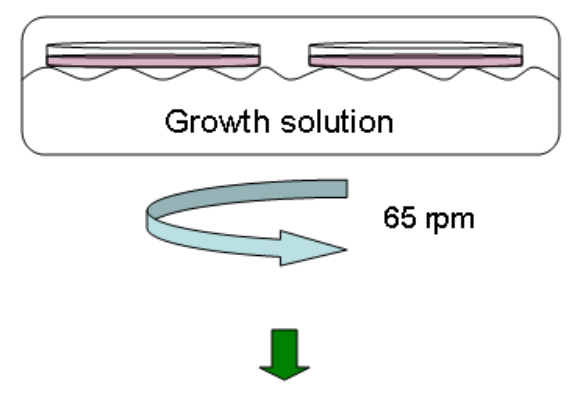

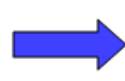

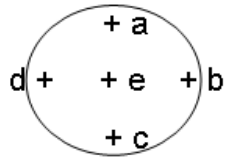

B)

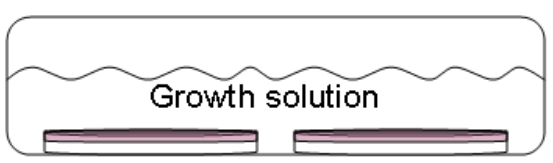

$65 \mathrm{pm}$

\begin{tabular}{|c|c|c|c|}
\hline $\mathrm{t}=1.5 \mathrm{~min}$ & $\mathrm{t}=1.75 \mathrm{~min}$ & $\mathrm{t}=2 \mathrm{~min}$ & $\mathrm{t}=2.25 \mathrm{~min}$ \\
\hline $38.1 \pm 1$ & $44.5 \pm 1$ & $44.9 \pm 1$ & $55.7 \pm 1$ \\
\hline $34.4 \pm 1$ & $42.0 \pm 1$ & $44.6 \pm 1$ & $50.8 \pm 1$ \\
\hline $30.4 \pm 1$ & $35.9 \pm 1$ & $37.3 \pm 1$ & $44.0 \pm 1$ \\
\hline $36.9 \pm 1$ & $36.3 \pm 1$ & $49.9 \pm 1$ & $56.9 \pm 1$ \\
\hline $43.0 \pm 1$ & $49.8 \pm 1$ & $48.8 \pm 1$ & $59.8 \pm 1$ \\
\hline
\end{tabular}

\begin{tabular}{|l|l|l|l|}
\multicolumn{1}{c}{$t=1.5 \min$} & $t=1.75 \min$ & $t=2 \min$ & $t=2.25$ \\
\hline $51.3 \pm 1$ & $63.3 \pm 1$ & $49.0 \pm 1$ & $25.5 \pm 1$ \\
\hline $41.8 \pm 1$ & $48.8 \pm 1$ & $64.6 \pm 1$ & $51.7 \pm 1$ \\
\hline $27.0 \pm 1$ & $29.8 \pm 1$ & $36.5 \pm 1$ & $45.4 \pm 1$ \\
\hline $24.7 \pm 1$ & $33.8 \pm 1$ & $50.9 \pm 1$ & $75.9 \pm 1$ \\
\hline $31.3 \pm 1$ & $28.1 \pm 1$ & $40.3 \pm 1$ & $65.6 \pm 1$ \\
\hline
\end{tabular}

Figure 56. Floating technique A) resulted in films with consistently $25 \%$ less thickness at the center while the settling technique B) produced variable film thicknesses. 
The settling technique apparently produced a more random pattern on the film thicknesses across a one-inch wafer. Furthermore, this technique generated more issues, such as the formation of air bubbles on the surface that led to holes on the film. The thicker edge phenomenon in the plated gold films was studied by Okinaka et al. [74] in 1974. In their study, they used different growth solution mixture and aimed for thicker films (micrometer range). They reported thicker films were obtained in the edge region compared to the center despite different agitation techniques used. Their results are shown in Figure 57. In their study, the distance between the center and the edge for a 4 $\mu \mathrm{m}$ thick gold film was $75 \mu \mathrm{m}$. The thickness difference from the center and the edge was $5 \%$. If this ratio of film thickness to edge-center distance is applied in this study, it means the measurement distance for a $44 \mathrm{~nm}$ film should be $825 \mathrm{~nm}$ and not $10 \mathrm{~mm}$.
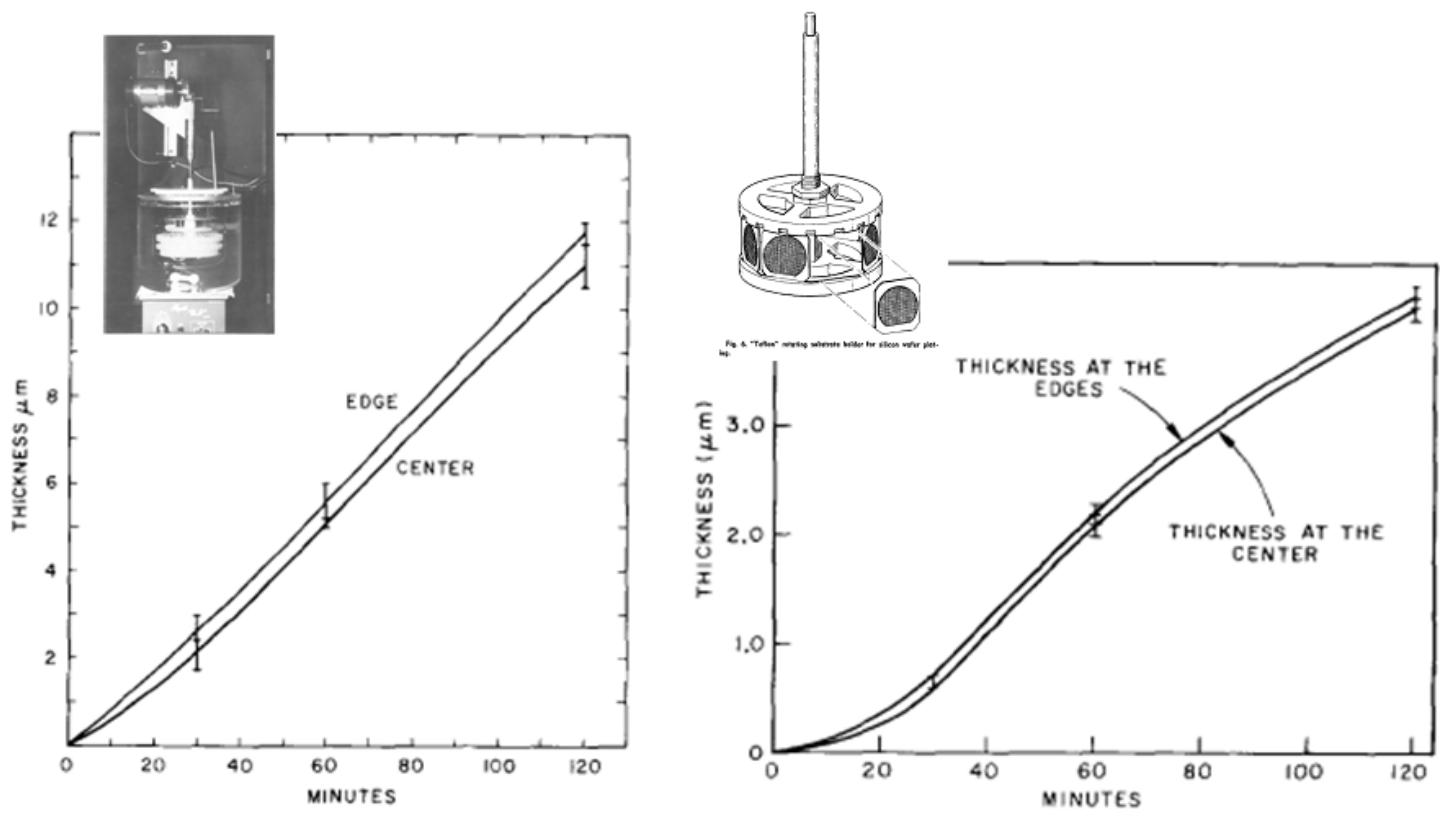

Figure 57. Thicker films at edges despite having different agitation methods as shown by Okinaka et al. [74] (reproduced by permission of The Electrochemical Society). 
Accordingly, it was decided that the distance between the edge and the center for the uniformity study should be reduced. Instead of $10 \mathrm{~mm}, 3 \mathrm{~mm}$ was defined as the center-edge distance. Within this new definition, it was found out that the plated gold films were indeed uniform. Gold film thicknesses of seven samples that were produced from two different batches are tabulated in Table 4. Measurements were done in five different spots (labeled 1-5) with 1 as the center point. All samples demonstrated excellent uniformity with a maximum of five percent tolerance. Therefore it was concluded that the floating technique was a better agitation method to produce uniform films in this research project. A shorter range $(3 \mathrm{~mm})$ was also applied as the distance reference in the film thickness measurements.

Table 4. Gold film thicknesses of seven samples from two batches $\left({ }^{\mathrm{a}}\right.$ and $\left.{ }^{\mathrm{b}}\right)$, measured at five points across a $3 \mathrm{~mm}$ radial area.

\begin{tabular}{|c|c|c|c|c|c|c|c|c|}
\hline \multirow[b]{2}{*}{ Sample } & \multirow{2}{*}{$\begin{array}{l}\text { Plating } \\
\text { time (min) }\end{array}$} & \multicolumn{5}{|c|}{ Gold film thickness (nm) } & \multirow{2}{*}{$\begin{array}{c}\text { Average } \\
\text { thickness } \\
(\mathrm{nm})\end{array}$} & \multirow{2}{*}{$\begin{array}{l}\text { Uniformity } \\
\text { tolerance }\end{array}$} \\
\hline & & 1 & 2 & 3 & 4 & 5 & & \\
\hline ja & $1 ' 45^{\prime \prime}$ & 34.2 & 35.4 & 35.6 & 36.1 & 36.8 & 35.62 & $1 \%$ \\
\hline iia & $2^{\prime} 00^{\prime \prime}$ & 37.4 & 36.8 & 35.7 & 37.4 & 38.0 & 37.06 & $1 \%$ \\
\hline $\mathrm{iija}^{\mathrm{a}}$ & $2^{\prime} 15^{\prime \prime}$ & 45.9 & 46.2 & 45.4 & 45.5 & 43.0 & 45.20 & $3 \%$ \\
\hline$i v^{b}$ & $2^{\prime} 15^{\prime \prime}$ & 35.8 & 35.9 & 39.2 & 37.0 & 38.3 & 37.24 & $5 \%$ \\
\hline$v^{b}$ & $2^{\prime} 30^{\prime \prime}$ & 43.1 & 41.8 & 43.4 & 44.1 & 41.7 & 42.82 & $3 \%$ \\
\hline$v^{b}$ & $2^{\prime} 45^{\prime \prime}$ & 43.7 & 46.6 & 45 & 43.1 & 45.7 & 44.82 & $5 \%$ \\
\hline$v_{i j}$ & $3^{\prime} 00^{\prime \prime}$ & 47.3 & 48.5 & 48.6 & 47.2 & 48.9 & 48.10 & $1 \%$ \\
\hline
\end{tabular}

\subsubsection{SPR Signal Generation}

After all conditions for the plating process were established, the final parameter to be optimized was the SPR signal generation. A comparison of SPR responses generated from electrolessly deposited gold film and evaporated gold film is presented in Figure 58. 
The plasmon depth from the electrolessly deposited gold is better than that from the evaporated gold. The width, however, is inferior compared to the evaporated gold. This occurrence might be as a result from the roughness of the film. In general, evaporated gold has a very low RMS value. The evaporated gold films produced with the vacuum chamber at IBM ARC have RMS values ranging from 0.5 to $1 \mathrm{~nm}$, while the RMS values for electroless gold range from 4 to $6 \mathrm{~nm}$. As for the resonance angle difference, it is likely due to a slight difference in the film thicknesses. Gold film thickness and roughness are known to greatly impact the generation of SPR signals [38].

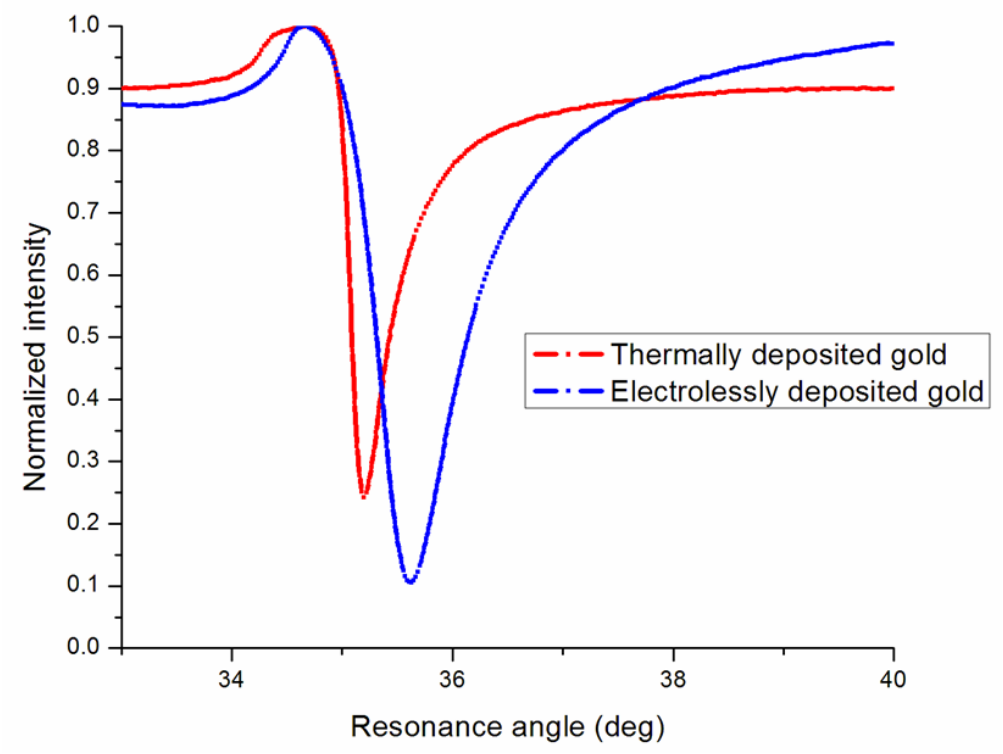

Figure 58. SPR curves of electrolessly deposited gold versus thermally deposited gold.

The overall procedure also demonstrated that SPR signal generation was reproducible. Figure 59 shows four SPR signals generated from three different electroless plated gold films and one evaporated gold film. The signals from two electroless gold films, labeled as 1 and 3, were located at the same resonance angle and slightly differed in the intensity depth. The intensity depth of the SPR signals from these 
two electroless plated gold films was higher compared to the evaporated gold signal. However, the width of the electroless gold plasmon was wider than the evaporated gold. On the other hand, a comparable width to the evaporated gold signal was shown by the electroless gold sample 2. However, the intensity depth was lower and the resonance angle shifted to a higher angle. Therefore, to achieve a good SPR signal from an electroless gold film, the intensity depth and plasmon width have to be compromised.

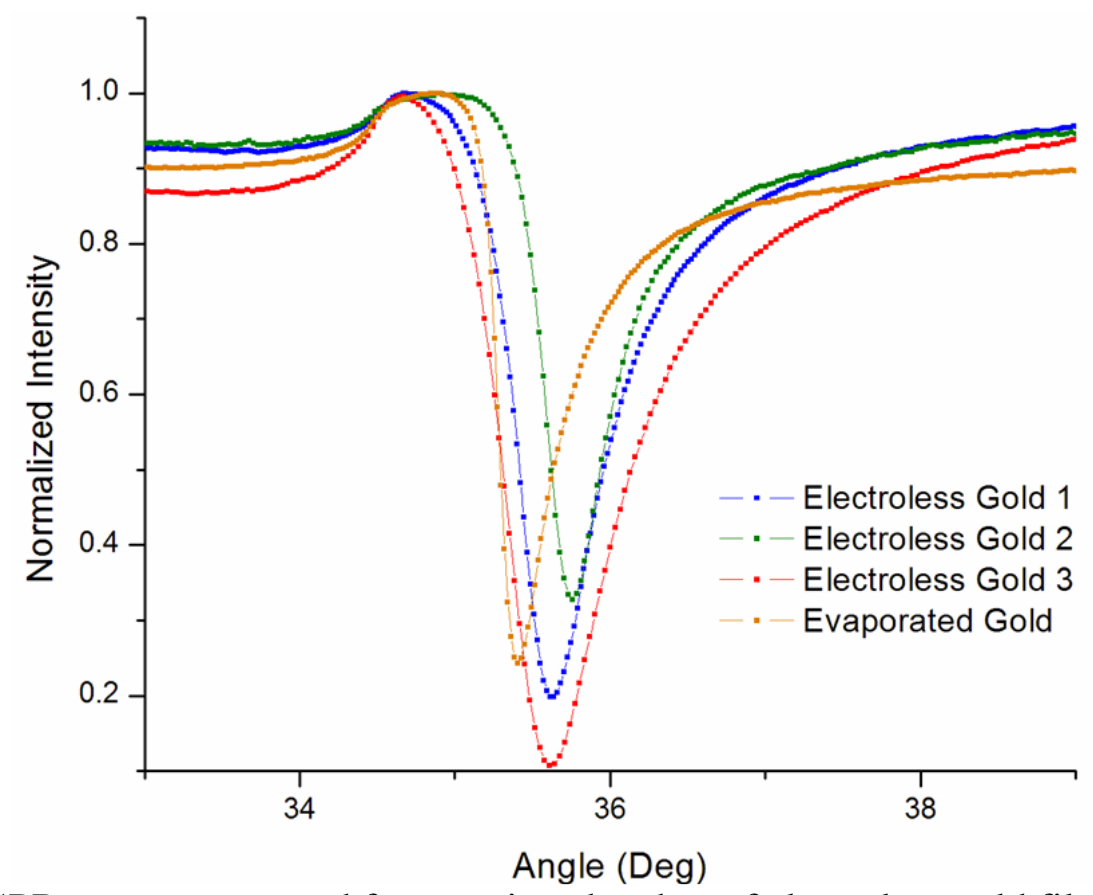

Figure 59. SPR curves generated from various batches of electroless gold films versus evaporated gold film.

\subsection{Comparison of Different Adhesive Templates}

\subsubsection{APTMS, PAH, and Star Polymer PS-DMAEMA Comparison}

The use of different adhesive templates was also explored in this work as a comparison study. APTMS and PDDA have been reported to demonstrate a good efficacy in promoting gold film growth [24-26]. Thus, a direct comparison with these 
templates can reflect the efficacy of star polymers in promoting gold film growth. PAH was used instead of PDDA in this project. Although they are slightly different in structure, both are categorized as amine-functionalized linear polymers. The morphologies of APTMS, PAH, and star polymer layer are presented in Figure 60. Figure 60A) and B) refer to the APTMS layers deposited via vapor deposition and solution deposition, respectively.
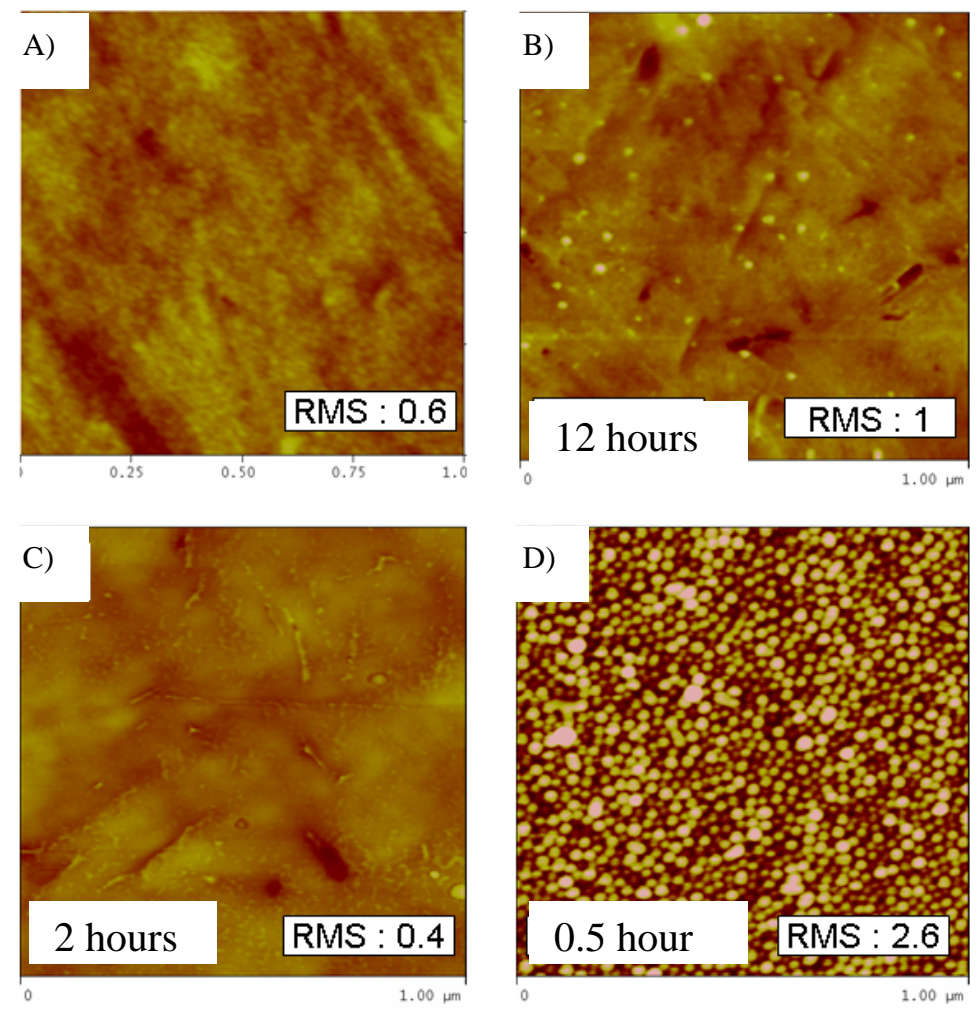

Figure 60. Morphology of different adhesive templates as characterized by AFM $(1 \mu \mathrm{m} \mathrm{x}$ $\mu \mathrm{m})$ : A) APTMS via vapor deposition, B) APTMS via solution deposition, C) PAH via solution deposition, and D) star polymer via solution deposition.

The APTMS deposition in solution state was done in 12 hours as suggested by Lei et al. [24], while a 2 hour deposition time was used for PAH [25]. The star polymer deposition process was carried out in 30 minutes. After the adhesive layers were 
deposited on the substrates, these substrates were immersed into a gold seed solution for one hour. Previous studies showed that the gold seed deposition usually took more than 16 hours to complete. From Figure 61, it can be seen that in one hour, star polymer templates developed a higher absorption intensity compared to APTMS and PAH. This suggests that star polymers exhibit a higher attachment force towards gold seeds.

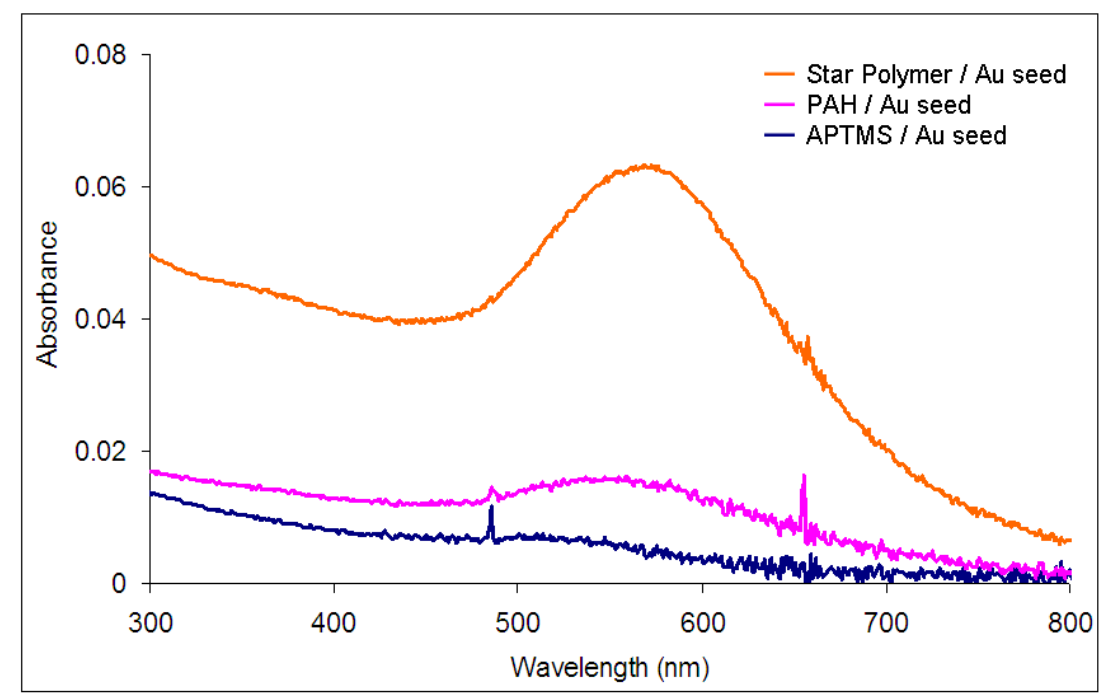

Figure 61. Various absorbance levels are shown by different templates (APTMS, PAH, and star polymer) at one hour seeding time.

The AFM analysis was then undertaken to evaluate the seed deposition. The gold seeds were more clearly observed in the APTMS-templated and PAH-templated substrates, as shown in Figure 62. The flatter feature of the APTMS or PAH layer made it easier to distinguish spherical gold seeds. Compared to the star polymers that formed closely-packed spherical structures, the spherical gold seeds were more difficult to observe. However, the inflated state of star polymers after the exposure to the gold seed implied that gold seed attachment had occurred. 

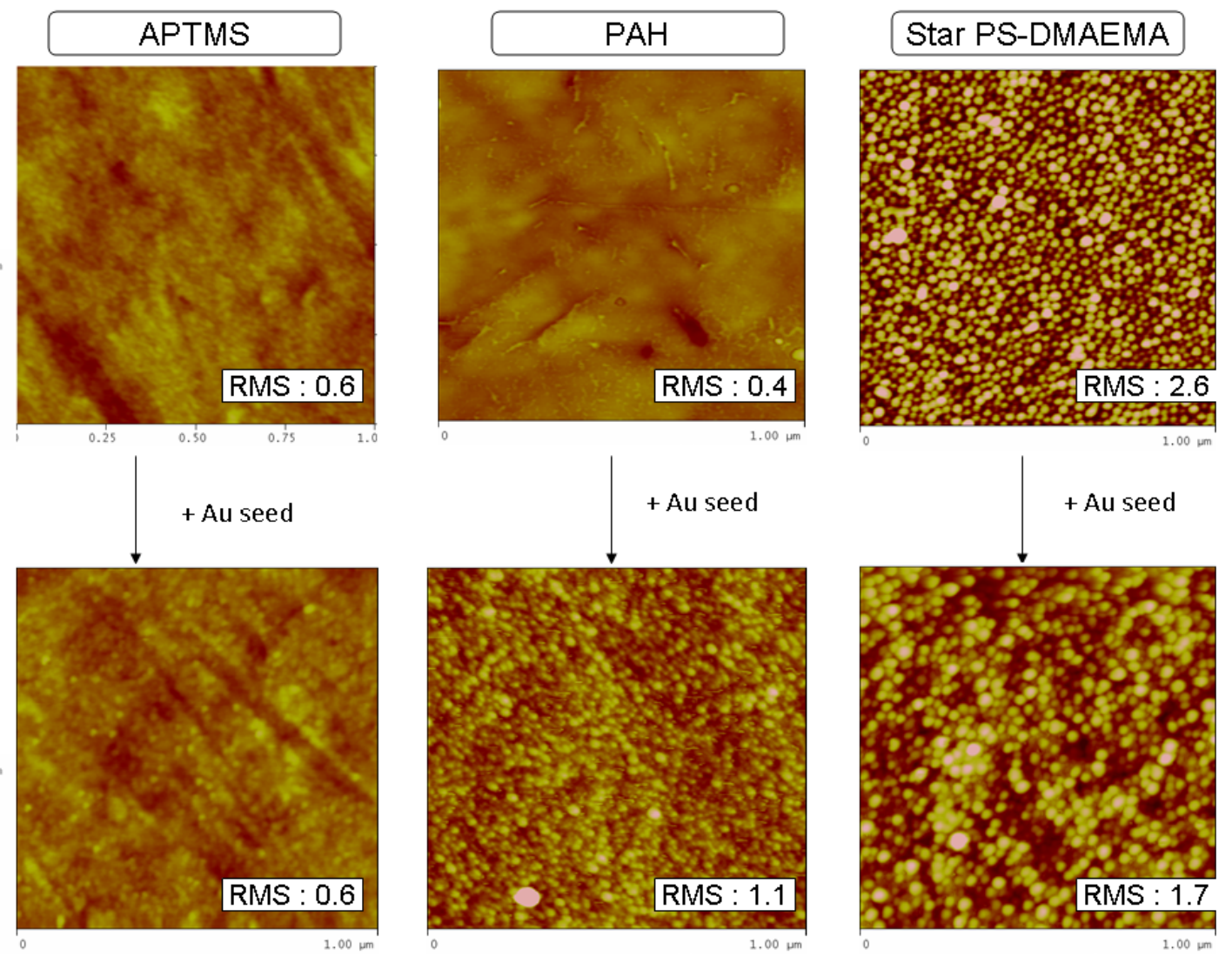

Figure 62. AFM images of gold seed deposited on different adhesive templates (APTMS, PAH, and star polymer PS-DMAEMA).

After seeded layers had been formed for APTMS-templated, PAH-templated, and star polymer-templated substrates, these substrates were exposed to a growth solution containing $0.5 \% \mathrm{w} / \mathrm{v}$ gold (III) chloride and hydroxylamine hydrochloride. The first experiment was performed to compare the gold films grown on all templates that were deposited at their optimal conditions (as reported in the published articles for APTMS and $\mathrm{PAH})$. The second experiment was carried out to compare the gold films grown on all templates that were deposited based on the conditions set forth by the star polymer template. 
The optimal conditions for each step in the first experiment are described in Table 5. For APTMS-based experiments, a substrate was immersed in the APTMS solution for 12 hours and then annealed at $120{ }^{\circ} \mathrm{C}$ for 30 minutes. The substrate was then immersed in the gold seed solution for 16 hours. As for the PAH-based experiments, a substrate was immersed in the PAH solution for 2 hours and then exposed to the gold seed solution for 20 hours. In the case of the star polymer-based experiments, the templating and seeding time were set at 30 and 60 minutes, respectively. All of these substrates were then put in a freshly made $0.5 \% \mathrm{w} / \mathrm{v}$ gold (III) chloride and hydroxylamine hydrochloride mixture for 3 minutes.

Table 5. Conditions applied throughout the whole process for APTMS, PAH, and star polymer comparison study.

\begin{tabular}{|c|c|c|c|c|}
\hline Template & Templating & Annealing & Seeding & Growth \\
\hline APTMS & 12 hours & 0.5 hour at $120^{\circ} \mathrm{C}$ & 16 hours & 3 min \\
\hline Star Polymer & 0.5 hour & - & 1 hour & 3 min \\
\hline PAH & 2 hours & - & 20 hours & 3 min \\
\hline
\end{tabular}

In order to determine film thickness and uniformity, the resulting gold films were analyzed by RBS. Measurements were done in five different spots with four points radially separated from the center point by $3 \mathrm{~mm}$. The film thicknesses are tabulated in Table 6. The average thickness of each film was in the range of $60 \mathrm{~nm}$ with a standard deviation value of 2.41. This standard deviation is assumed to be caused by the time differences from the substrates being pulled out of the growth solution. In terms of film uniformity, all templates generated uniform films across one-inch wafers by maintaining uniformity tolerance under $10 \%$. 
Table 6. Gold film thicknesses resulting from three different templates. Measurement was carried out in five different spots on the films. * Tol: maximum thickness difference of each spot compared to average thickness.

\begin{tabular}{|c|c|c|c|c|c|c|c|}
\hline \multirow{2}{*}{ Template } & \multicolumn{5}{|c|}{ RBS Thickness (nm) } & \multirow{2}{*}{ Avg (nm) } & \multirow{2}{*}{ Tol *(\%) } \\
\cline { 2 - 6 } & 1 & 2 & 3 & 4 & 5 & & 10 \\
\hline APTMS & 64.3 & 60.9 & 59.7 & 59.8 & 66 & 62.14 & 10 \\
\hline Star Polymer & 60.6 & 64.6 & 72.3 & 65.4 & 63 & 65.18 & 10 \\
\hline PAH & 61.1 & 58.5 & 61.2 & 56.9 & 64.4 & 60.42 & 10 \\
\hline
\end{tabular}

Based on the AFM results in Figure 63, the gold film morphology for all templates (APTMS, PAH, and star polymer) are similar in terms of the grain size/shape and how the grain was closely packed in a non-ordered structure. The lowest RMS value was shown by the star polymer-templated gold film despite the fact that the star polymer layer had a higher RMS value compared to APTMS or PAH. This demonstrates that the wavy surface of star polymer layer as the initial layer did not lead to a rough gold film surface.
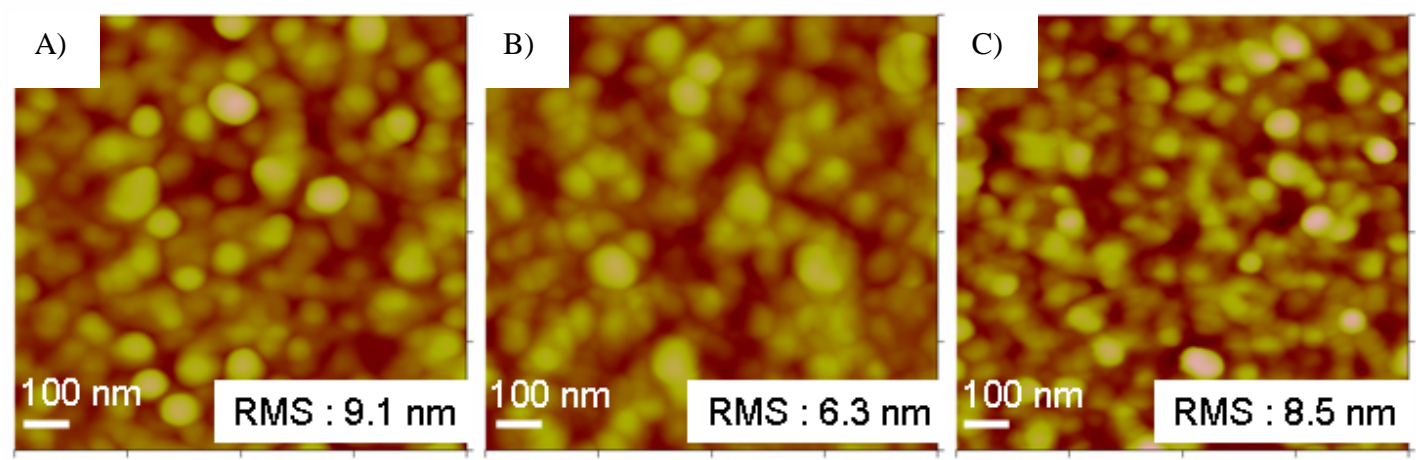

Figure 63. Gold film as grown on: A) APTMS, B) star polymer, and C) PAH in 3 min plating time. The process conditions and resulting film thicknesses of each film can be found in Table 5 and 6 , respectively. 
Based on the AFM analysis, the star polymer-templated gold films were shown to be as good as the APTMS-templated and PAH-templated gold films in terms of film morphology and roughness. Another film property of interest was mechanical stability, particularly on how strong the star polymer-templated gold adhered to the substrate compared to APTMS-templated or PAH-templated gold considering its short processing time. For clarification, scratch tests were performed. The scratch test is a common method to determine film delamination. However, the thicknesses of the measured films are usually in the micrometer range. In this study, a nanoindenter was specifically used to perform the scratch tests because the measured films were very thin (less than 100 $\mathrm{nm})$.

Electroless gold films with different types of adhesive templates (APTMS, PAH, and star polymer) and evaporated gold film with $\mathrm{Cr}$ as an adhesive layer were independently tested with $150 \mu \mathrm{N}$ normal load setting at seven different locations. The normal load setting was chosen based on the nanoindentation results that were obtained prior to the scratch tests. The nanoindentation results of the electroless gold and the evaporated gold films showed that $150 \mu \mathrm{N}$ was sufficient to induce physical contact up to $20 \mathrm{~nm}$ (data is not shown here). Considering the film thicknesses for the tested gold films were was in the range of $60 \mathrm{~nm}$, the effect of $20 \mathrm{~nm}$ contact depth should propagate way deeper $(\sim 60 \mathrm{~nm})$ where the interface of adhesive materials and gold was located.

For each scratch test measurement (at $150 \mu \mathrm{N}$ normal load), the lateral force was plotted versus time. The lateral force magnitude is inversely proportional to the mechanical stability of a film. Despite the type of the adhesive materials, the electroless 
gold films displayed a similar lateral force trend as shown in Figure 64. This indicated that regardless the short processing time that star polymer experienced, the quality of the induced gold film was equal to that of APTMS or PAH which required a longer processing time. Regardless of which adhesive templates were used for the electroless gold film, the electroless gold films were still inferior to the evaporated gold films. The method of deposition (evaporation and chemical reduction) might have been a contributing factor and further studies would be required to improve the mechanical properties of the electroless gold.

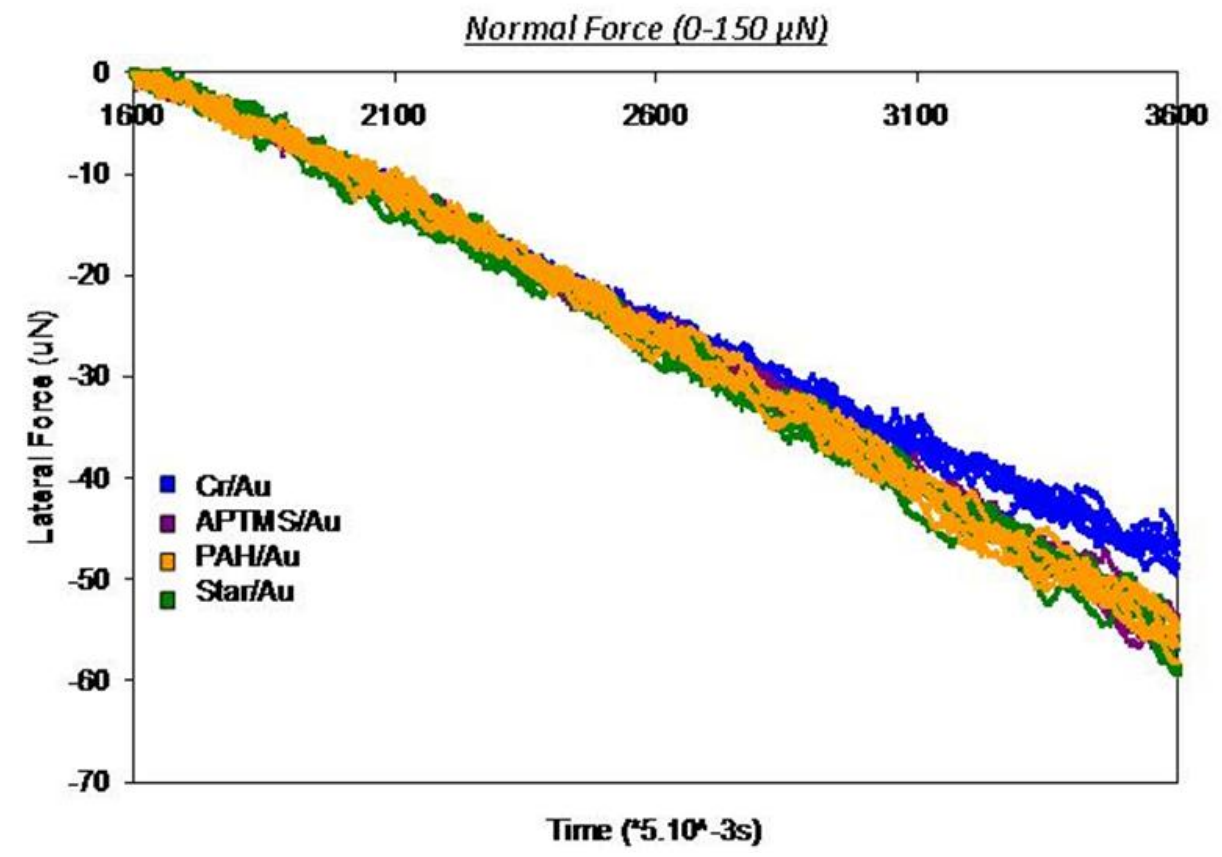

Figure 64. Lateral force measurement as a method to compare mechanical stability of star polymer-templated gold film to that of evaporated gold film and APTMS-templated or PAH-templated gold film.

As demonstrated previously, one apparent advantage of the process incorporating star polymer is the short templating and seeding time. A question then emerged on how 
other templates (APTMS and PAH) behaved if star polymer processing time was applied. To answer this question, a second experimental setup was carried out. The templating time was set at 30 minutes while the seeding time was set at one hour. No annealing was performed for the films after the templating process. After all substrates had been subjected to a seeding process, a mixture of freshly made $0.5 \% \mathrm{w} / \mathrm{v}$ gold (III) chloride and hydroxylamine hydrochloride was brought into contact with the substrates. The plating time was set at 3 minutes for all substrates. The resulting gold films were then analyzed using RBS spectroscopy. Measurements were done in five different spots with four points radially separated from the center point by $3 \mathrm{~mm}$. The data is summarized in Table 7.

Table 7. Gold film thicknesses resulting from three different templates under the conditions applied to star polymer ( 0.5 hour templating and 1 hour seeding with 3 min plating time). Measurement was carried out in five different spots on the films. ${ }^{*}$ Tol: maximum thickness difference of each spot compared to average thickness.

\begin{tabular}{|c|c|c|c|c|c|c|c|}
\hline \multirow[b]{2}{*}{ Template } & \multicolumn{5}{|c|}{ RBS Thickness (nm) } & \multirow[b]{2}{*}{ Avg (nm) } & \multirow[b]{2}{*}{ Tol (\%) } \\
\hline & 1 & 2 & 3 & 4 & 5 & & \\
\hline APTMS & 64.9 & 60.8 & 55.9 & 63.5 & 60.4 & 61.10 & 10 \\
\hline Star Polymer & 60.6 & 64.6 & 72.3 & 65.4 & 63 & 65.18 & 10 \\
\hline PAH & 47.4 & 47.7 & 49.5 & 54.6 & 45.7 & 48.98 & 12 \\
\hline
\end{tabular}

The RBS results showed that APTMS and star polymer templates generated gold films with similar average thicknesses. The uniformity levels of both films were below $10 \%$. The PAH, on the other hand, resulted in a fairly poor film with a lower film thickness and uniformity. These results imply that 30 minute templating time and one hour seeding time were essentially sufficient for the APTMS to generate a good gold 
film, but they were insufficient for the PAH. The AFM results in Figure 65 also revealed that the PAH-templated gold film had a rougher film compared to the APTMS-templated and star polymer-templated gold films.
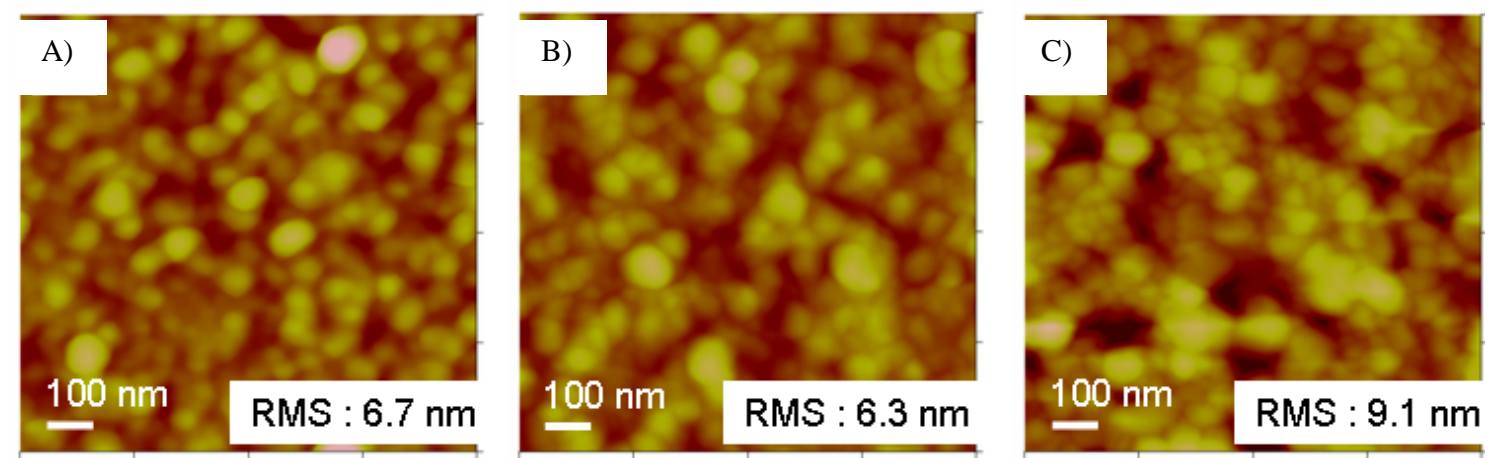

Figure 65. Gold film as grown on: A) APTMS, B) star polymer, and C) PAH under the conditions applied to star polymer ( 0.5 hour templating and 1 hour seeding with 3 min plating time). The resulting film thicknesses of each film can be found in Table 7.

\subsubsection{Star Polymer PS-NH $\mathrm{NH}_{2}$ as Template}

The other template that was explored in this project was the star polymer PS- $\mathrm{NH}_{2}$. The distinguishable parameter that differentiates star polymer PS-DMAEMA and PS$\mathrm{NH}_{2}$ is the solvent compatibility. PS-DMAEMA can be dissolved in an aqueous solution while PS- $\mathrm{NH}_{2}$ can only be dissolved in organic solvents. The objective of this experiment was to show that although organic solvents were involved in the star polymer deposition, the dry state of the star polymer layer still supported the gold seed attachment and the growth of gold particles. According to Bonifacio and Chang, the suitable solvent for the star polymer PS- $\mathrm{NH}_{2}$ deposition is dichloromethane (DCM) followed by a THF wash $[57,58]$. Therefore this solvent mixture was also used for the initial experiment. A pre-treated (with piranha solution) glass substrate was immersed into star polymer PS$\mathrm{NH}_{2}$ solution for ten minutes. A THF wash was then performed three times to remove 
any unbound materials. The deposition was probed by the AFM. As seen in Figure $66 \mathrm{~A})$, the star polymer PS- $\mathrm{NH}_{2}$ layer did not form a uniform and contiguous film due to dewetting problems. Although this dewetting issue was acknowledged, it was decided to expose this substrate to a gold seed solution. Interestingly, the gold seeds attached to the star polymer layer as shown in Figure 66B). Following the growth step, clumps of the enlarged gold particles were spotted randomly as shown in Figure 66C).
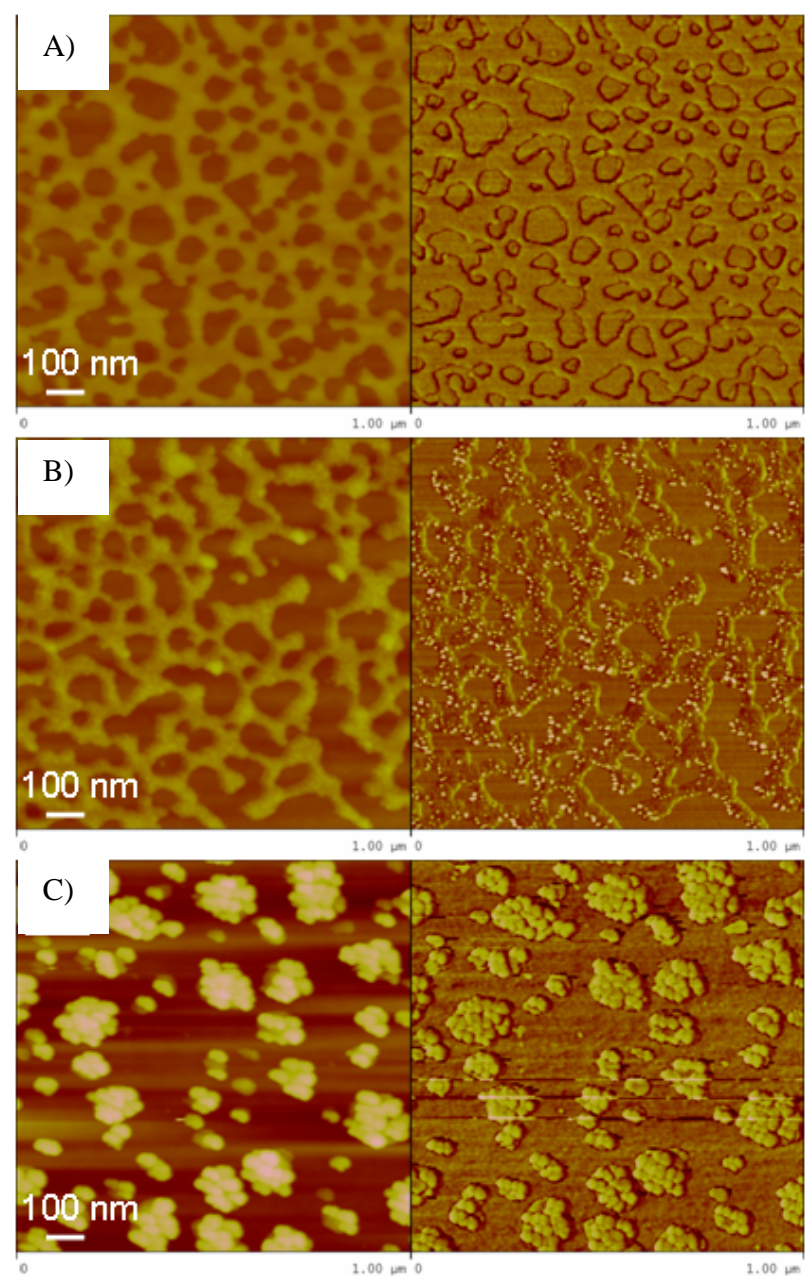

Figure 66. PS-NH2 layer (DCM as the dissolving solvent) before and after an exposure to a gold seed solution and a growth solution. A) before exposure to a gold seed solution, B) after being exposed to a gold seed solution, and C) after being exposed to a growth solution. Z height: $50 \mathrm{~nm}$. 
In order to make a uniform and contiguous star polymer PS-NH 2 layer, solvent choice was re-evaluated and toluene was used to dissolve the star polymer. Apparently, toluene assisted the formation of a uniform and contiguous film of the star polymer PS$\mathrm{NH}_{2}$ as shown in Figure 67A). The star polymer in dry state also developed an attachment of gold seeds as shown in Figure 67B). The enlarged gold particles were more closely packed, as shown in Figure 67C). Therefore it was concluded that the star polymer PS- $\mathrm{NH}_{2}$ in toluene could be used as a template in the electroless gold plating, however, the resulting film was not as contiguous as the star polymer PS-DMAEMA.
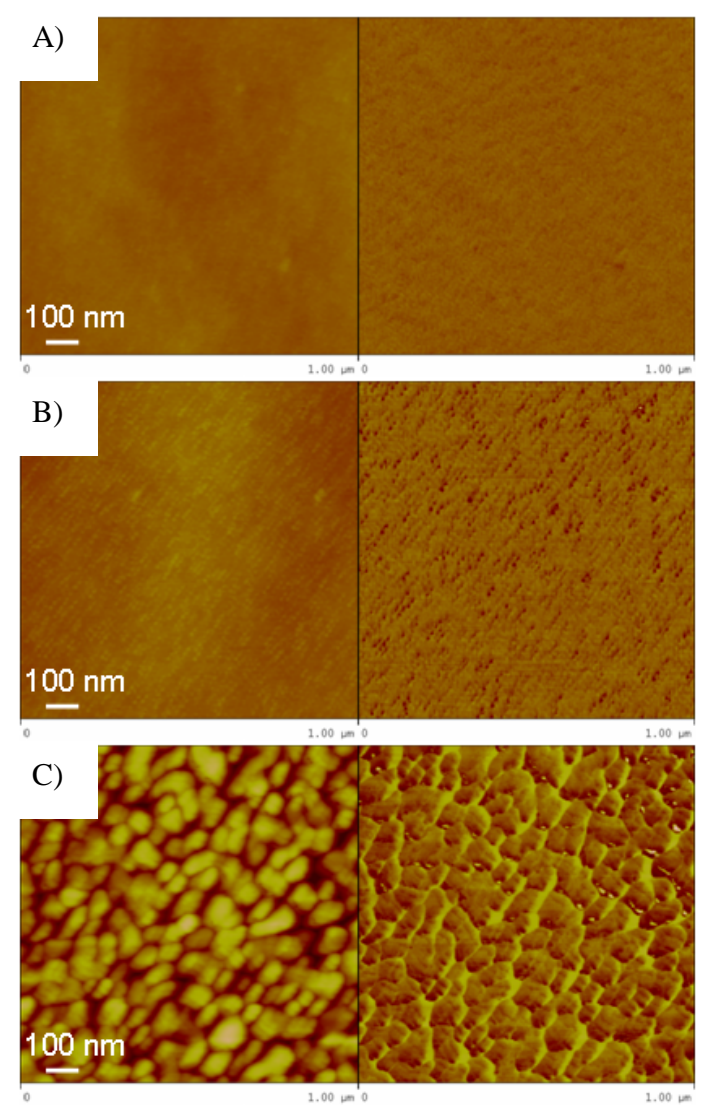

Figure 67. PS-NH2 layer (toluene as the dissolving solvent) before and after an exposure to a gold seed solution and a growth solution. A) before exposure to a gold seed solution, B) after being exposed to a gold seed solution, and C) after being exposed to a growth solution. Z height: $30 \mathrm{~nm}$. 


\subsection{D Gold Nanoshell Synthesis}

As mentioned previously, gold nanoshells have emerged as a potential candidate in cancer imaging and therapy due to their strong scattering and/or absorption in the NIR window. Other studies have shown that the close proximity ( $\sim 10 \mathrm{~nm}$ or greater $)$ of molecular chromophores to a metal surface could lead to a fluorescence enhancement by a 100-fold [75]. A direct attachment or a less than $5 \mathrm{~nm}$ proximity of chromophores to a metal surface, on the other hand, can quench the fluorescence effect $[75,76]$. From the literature, the process to put chromophores at the proper distance from the gold nanoparticles was tedious. A conjugation of chromophores to a spacer layer was required, and chromophores were able to be positioned only at the outer layer of the gold nanoparticles [76].

By utilizing star polymer as a template, chromophores can be effortlessly embedded inside the star polymer via self-assembled occlusion complex formation. Proximity can easily be adjusted by utilizing different sizes of star polymers. The outer layer of gold shell can be exploited for any desired bio-conjugation or functionalization. Other materials such as drugs can be embedded inside the star polymer leading to an integrated form of imaging contrast, drug-delivery system, and photothermal ablation agent. Furthermore, the feasibility of star polymer as a template in gold thin film formation was demonstrated. Therefore, an adaptation of this technique to generate a star polymer-templated gold nanoshell was of interest. The synthetic process for this adaptation is presented in Figure 68. 


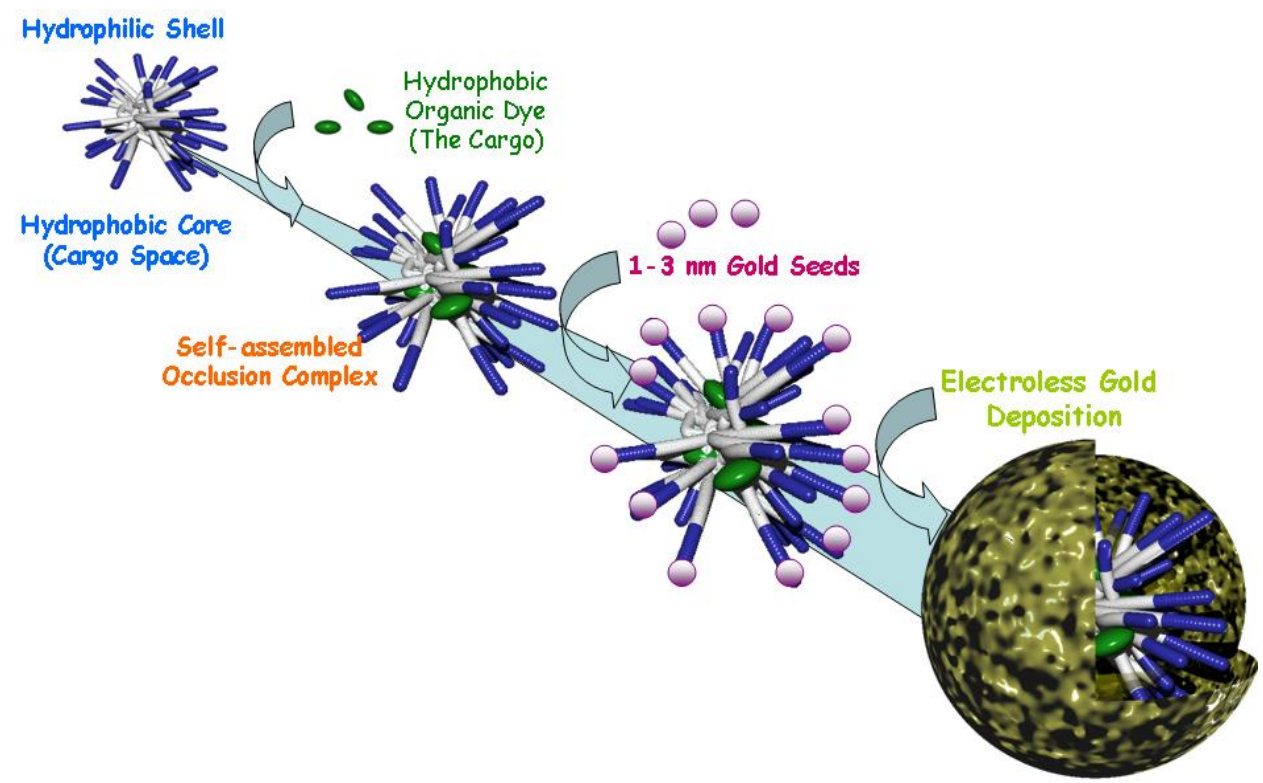

Figure 68. Illustration of gold nanoshell formation utilizing star polymer as a template.

Star polymers with an average hydrodynamic diameter of $25 \mathrm{~nm}$ (in water) were used as templates in this investigation. The smaller the seed size, the more coverage that can be attained on the surface of the star polymer. Therefore, a different approach to synthesize gold seeds was applied by using THPC as the reducing agent in gold (III) chloride reduction. This route had been reported to produce negatively charged gold colloids $1-3 \mathrm{~nm}$ in diameter $[16,68,69]$. A change in color solution from colorless to light brown was observed as soon as gold (III) chloride was added to the NaOH/THPC mixture. The color of the solution is shown in Figure 69. According to Shi et al. [16], the change in color indicates the formation of small gold nanoparticles. The formation of the gold nanoparticles was also tracked by a UV-visible spectrophotometer. A weak absorbance peak at $\sim 510 \mathrm{~nm}$ was also observed in the seed solution, as depicted in Figure 69. This peak is similar to that obtained by Park et al. [77] for their gold seed solution 
(1-3 nm gold/THPC). The synthesized gold seeds were further characterized by DLS to determine the particle size and distribution. The DLS results are presented in Figure 70. Based on these DLS data, $89.5 \%$ (\% weight) of the particles were $2.2 \mathrm{~nm}$ in diameter.

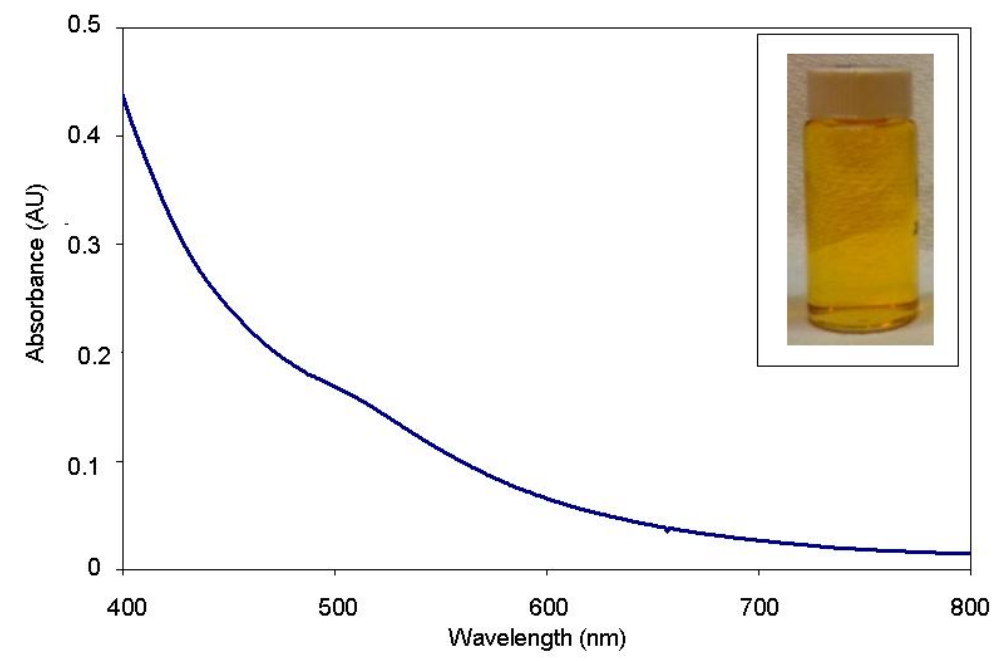

Figure 69. UV-visible absorption spectrum of gold/THPC (Inset: the light brownish color of gold/THPC solution).

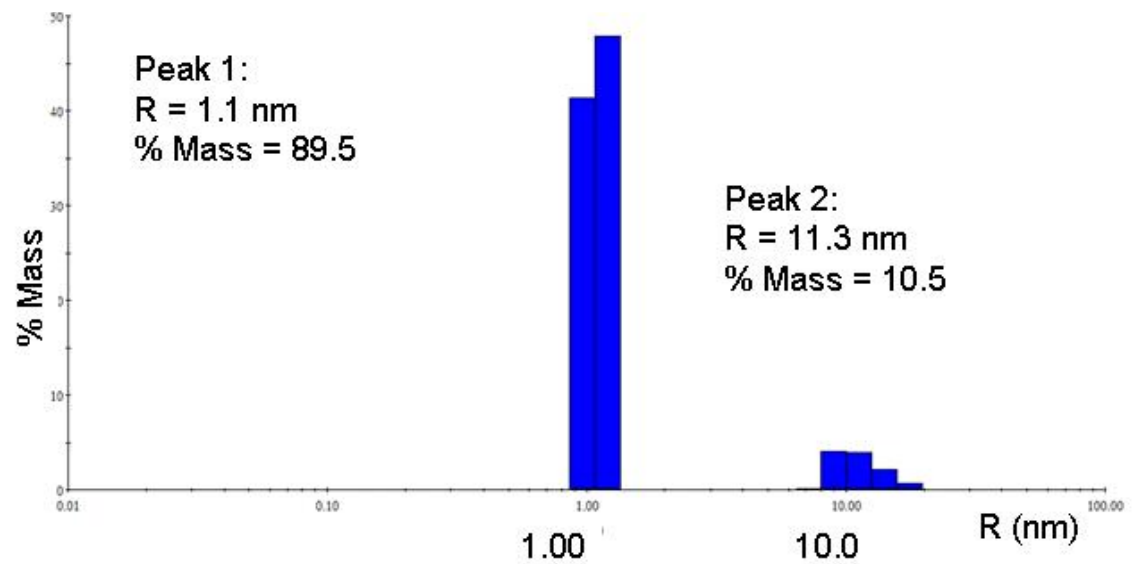

Figure 70. DLS data of the gold/THPC that was used as seed in gold nanoshell formation.

After the seed size was confirmed to be $1-3 \mathrm{~nm}$, selective precipitation of gold seeds by centrifugation was performed to ensure the attachment of these seeds to the 
amine groups of the star polymers and to remove any unbound seeds to avoid nonspecific growth. Using this process, solutions of the gold seeds produced a significant amount of black precipitate after centrifugation (4,000 rpm, 60 minutes) in the presence of THF. The presence of THF presumably reduced the solubility of the gold seeds, allowing them to be isolated by centrifugation. In contrast, when a mixture of $5 \mathrm{~mL}$ gold seeds and $5 \mathrm{~mL}$ star polymer solution was centrifuged at $4,000 \mathrm{rpm}$ for 60 minutes in the presence of THF, only a trivial amount of precipitate was observed. This finding indicated that the gold seeds were associated to the star polymers, and the association allowed them to be soluble in THF and prevented them from precipitating. To test this hypothesis, $15 \mathrm{~mL}$ gold seed solution was added to the previous mixture of star polymer and gold seeds. The new solution was centrifuged for a second time at 4,000 rpm for 60 minutes. At the end of the centrifugation process, black precipitates were visible at the bottom of the centrifuge tube. This precipitation suggested that an excessive amount of seeds was available in the solution, and the non-associated ones (with the star polymer) precipitated due to their insolubility in THF. These set of experiments indirectly demonstrated that the gold seeds were attached to the star polymers. The unbound seeds were able to be avoided by adding the right amount of seeds into the star polymer solution. In this case, the volume of $5 \mathrm{~mL}$ of seeds was shown to be the saturation limit for $5 \mathrm{~mL}$ star polymer solution. A more comprehensive study is required if the precise limit for gold-seed saturation is to be determined.

After the seeding step was established, the next step was to grow the gold seeds. The enlarged seeds would eventually coalesce and form a thin gold film at the outer layer 
of core materials as suggested by previous studies. As described in the materials and method chapter, the growth step was started by mixing gold-decorated star polymer with gold hydroxide. The addition of hydroxylamine hydrochloride completed the reduction process and led to the gold nanoshell formation. The change in the color of solutions from clear yellow to blue, as shown in Figure 71 (right), was one indication that the nanoshells had been formed. The blue color as a sign that solution contained gold nanoshells was also observed in prior studies $[17,20]$. The blue color of a material is typically due to its red light absorption (longer wavelength in the visible range). The UV-visible spectra of the blue solutions in Figure 71 (left) confirmed that they all absorbed light at the longer wavelength of the visible range $(700 \mathrm{~nm})$ and at the NIR range $(850-1,000 \mathrm{~nm})$. The longer wavelength absorption in this case corresponded to a more bluish color.
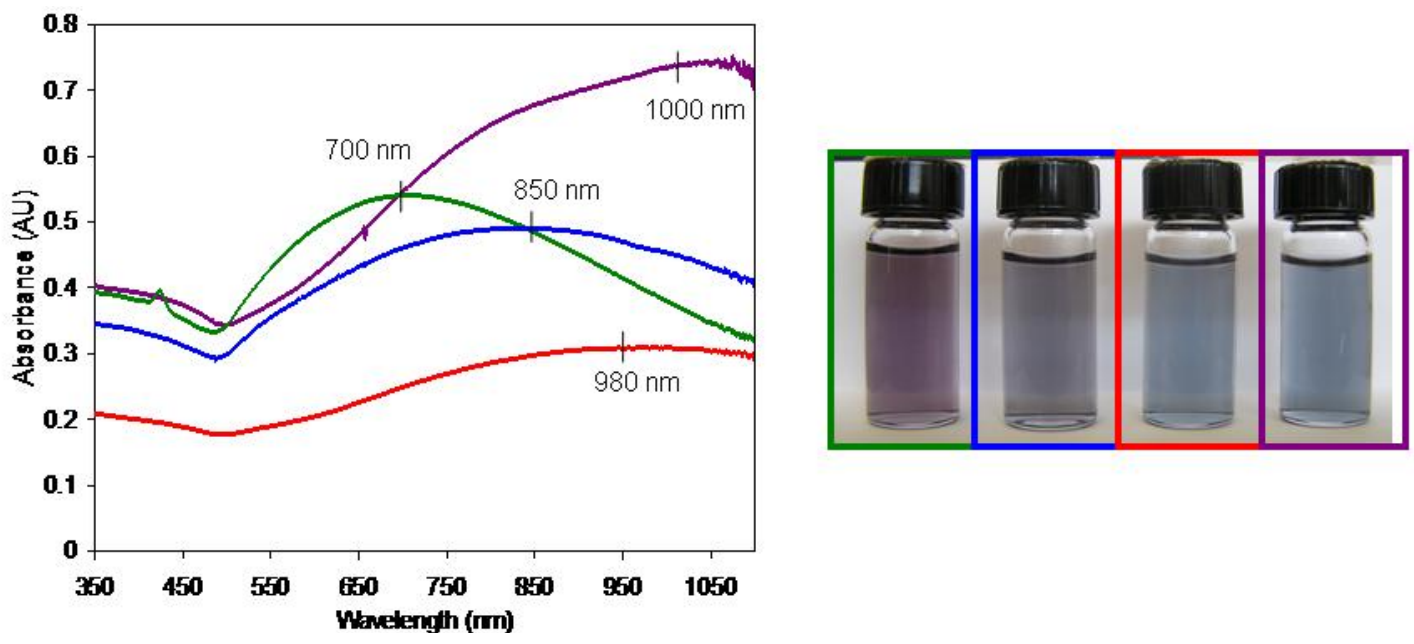

Figure 71. A tunable NIR absorption from different sizes of star polymer-templated gold nanoshells (left) and the corresponding color of solutions in the left image (right). 
To determine the final sizes of these particles, SEM analysis was performed. The SEM samples were prepared as described in the materials and method chapter. Briefly, the samples were spun down onto a clean silicon wafer and dried at $90{ }^{\circ} \mathrm{C}$ for one minute prior to the analysis. The SEM analysis revealed that the particles that displayed absorption peaks at 700, 850, 980, and 1,000 $\mathrm{nm}$ had a mean diameter of 90, 130, 200, and $220 \mathrm{~nm}$, respectively. The SEM images are presented in Figure 72.

A)

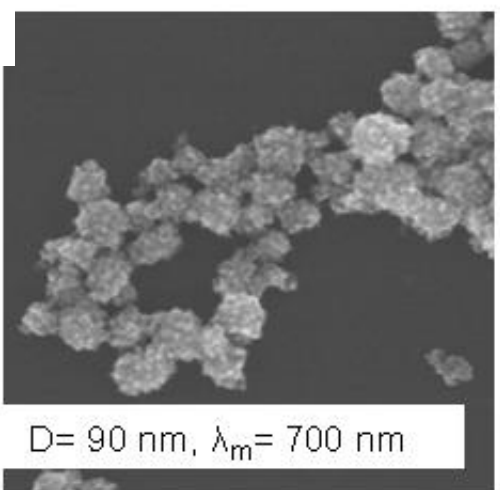

B)

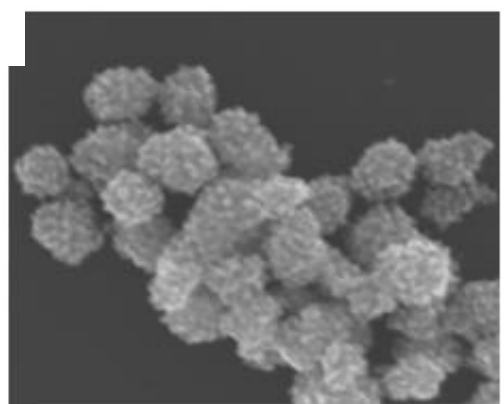

$\mathrm{D}=130 \mathrm{~nm}, \lambda_{\mathrm{m}}=850 \mathrm{~nm}$

$100 \mathrm{~nm}$

C)

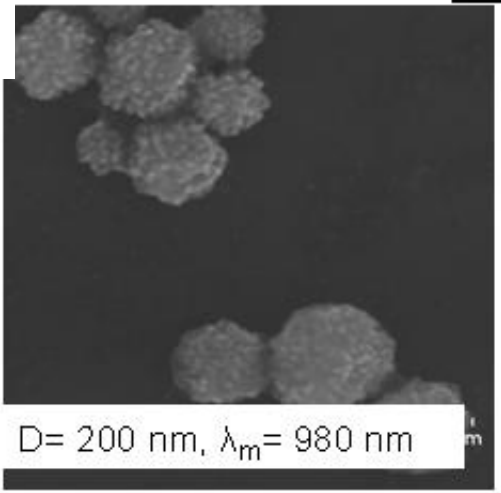

D)

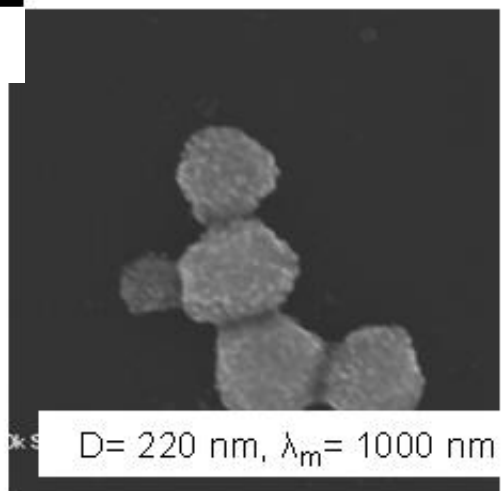

Figure 72. SEM images of different sizes of star polymer-templated gold nanoshells. D $=$ diameter, $\lambda_{\mathrm{m}}=$ absorption peak.

This result suggested that the absorption relied heavily on the final size of the particles. Larger particles drive the absorption to a longer wavelength. The size of the 
particles itself is a function of the amount of seeds and concentration of the growth solution. From the SEM images, it was obvious that the surface of these particles was not smooth. The same results were reported in previous studies, some of which focused on reducing this roughness issue in search of higher absorption or scattering cross-section. However, no attempt to reduce roughness was carried out in this work.

In order to get clean particles for SEM imaging as shown in Figure 72, a series of purification techniques were attempted. A non-purified (crude) sample usually carries excess reagents or by-product such as salts, as shown in Figure 73 (left). The first attempt to remove these salts was to filter the solution with a $0.45 \mu \mathrm{m}$ glass filter prior to spin-coating. However, the SEM analysis in Figure 73 (right) showed that this filtering process also removed the desired gold nanoshells. Presumably, the gold nanoshells were somehow interacted with one another leading to entanglement in the filter.
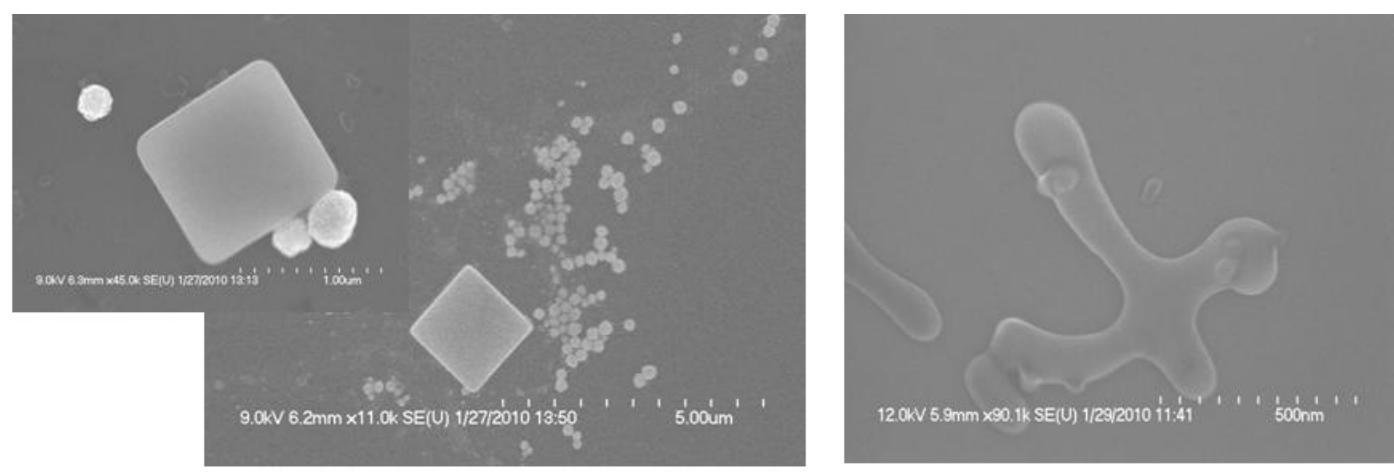

Figure 73. SEM images of a crude sample of star polymer-templated gold nanoshells with observable excess salts (left) and the crude sample after filtration with a $0.45 \mu \mathrm{m}$ glass filter (right).

Since filtering the samples was ineffective in cleaning the particles, dialysis was attempted. A crude sample was put into a 12-14,000 Da MWCO cellulose bag and 
subjected to a dialysis process against Millipore water for 24 hours. The dialysis seemed to remove the salts. However, some other materials surrounded the particles and formed a cloudy background, as shown in Figure 74B). In order to remove these background materials, a second dialysis step was performed. In this case, methanol was used as the solvent instead of Millipore water and the solution was stirred in the methanol for 24 hours. SEM analysis showed that the second dialysis successfully removed the background materials. The particles were clean as shown in Figure 74C).
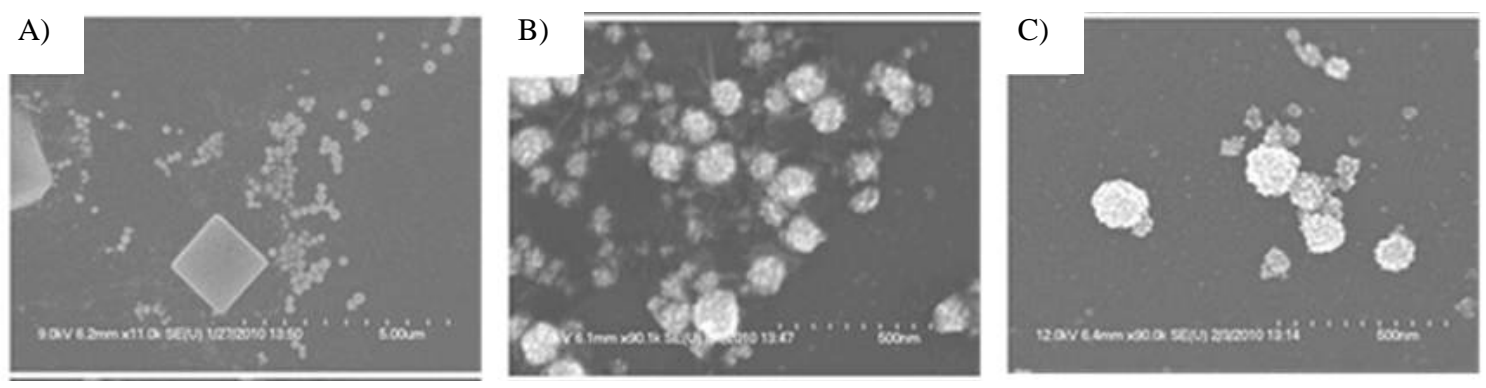

Figure 74. SEM images of a crude sample of star polymer-templated gold nanoshells: A) before dialysis, B) after 24 hours dialysis against Millipore water, and C) after 24 hours dialysis against Millipore water and 24 hours against methanol.

Although dialysis appeared to be an effective solution to clean the particles, the long dialysis time ( $2 \times 24$ hours) was considered to be a drawback. Thus, centrifugation was attempted. A crude sample was centrifuged at 4,000 rpm for one hour. The supernatant was removed and the pellet was redispersed in $4 \mathrm{~mL}$ of Millipore water. The redispersed solution was then subjected to SEM-sample preparation as described in the materials and methods chapter. SEM analysis of the redispersed pellet showed that onestep centrifugation effectively removed contaminants and/or salts. The particles were clean compared to the crude sample, as shown in Figure 75. Therefore, one-step 
centrifugation was decided to be the main purification technique to clean synthesized gold nanoshells in this work.
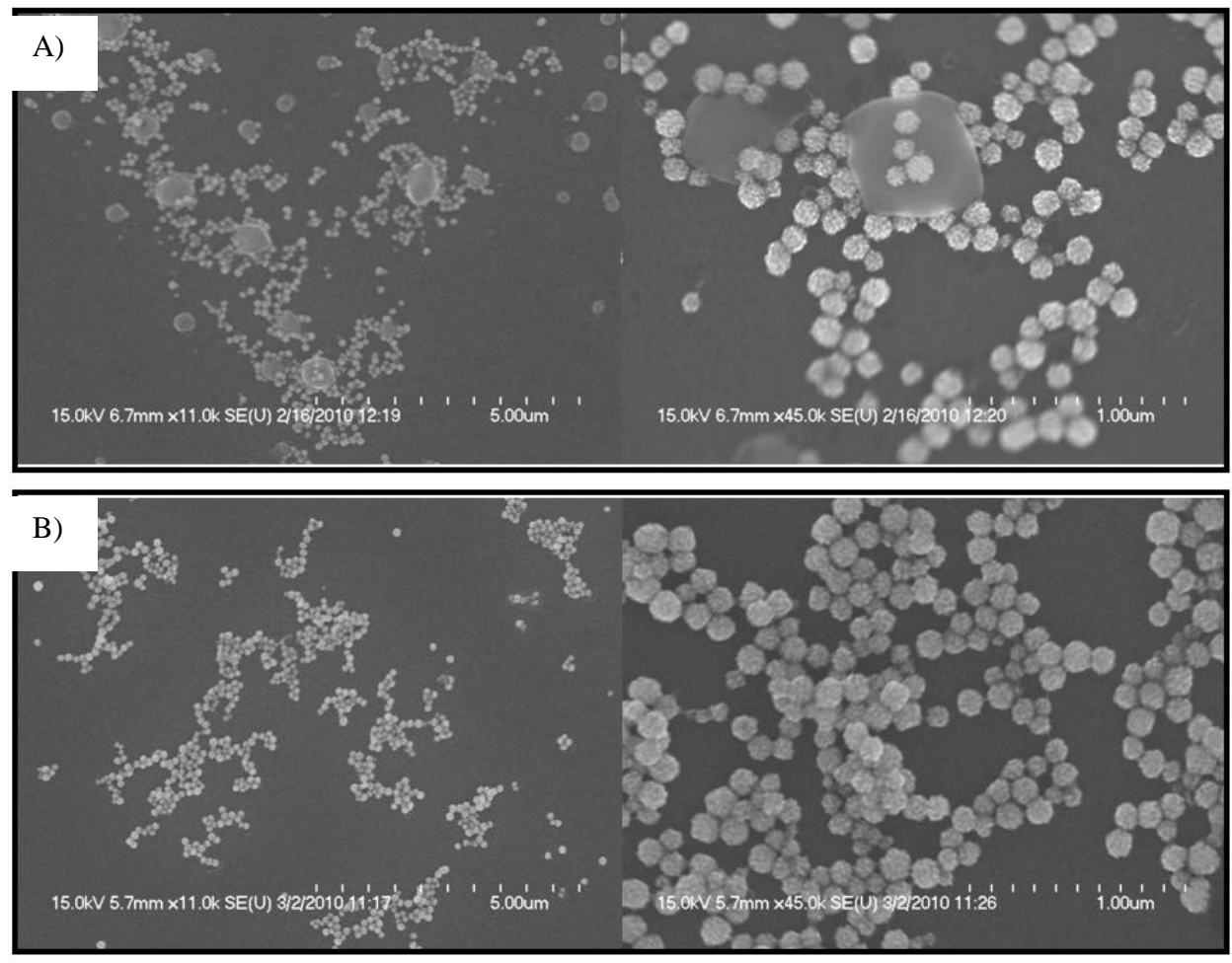

Figure 75. SEM images of a crude sample of star polymer-templated gold nanoshells: A) before centrifugation and B) after one-step centrifugation at 4,000 rpm for one hour (pellet was redispersed in Millipore water).

After establishing the purification technique, the next experiments were aimed to target a specific size of gold nanoshells. The size of nanoparticles significantly affects the particles intracellular uptake and distribution in the body $[78,79]$. The size of nanoparticles particularly affects passive delivery capability of nanoparticles in the tumor studies. Tumor vessels have a leaky endothelial structure with 100 to $780 \mathrm{~nm}$ gaps compared to 5 to $10 \mathrm{~nm}$ of normal vessels [79]. With the right size of particles, an enhanced permeability to tumor vessels and preferential accumulation in the tumor cells 
can be achieved. One study demonstrated that nanoshells with $130 \mathrm{~nm}$ in diameter easily passed through the walls of vessels and accumulated in the surrounding tumor tissues [78]. Therefore, gold nanoshells with a mean diameter of $100 \mathrm{~nm}$ were specifically targeted in this work to achieve similar function of passive delivery to tumor cells.

As shown previously, the size of gold nanoshells was able to be varied by adjusting the seed amount and growth solution concentration. However, the rate of the hydroxylamine hydrochloride addition was also observed to be important in synthesizing nanoshells with a certain size. Relatively enormous particles with $500 \mathrm{~nm}$ in diameter were obtained by slowly adding hydroxylamine hydrochloride. In addition, smaller particles with $200 \mathrm{~nm}$ diameter were also noticeable. With a slow addition, the resulting particle diameters were in the range of 100 to $250 \mathrm{~nm}$ with a high polydispersity level. In contrast, particles with an average diameter of $100 \mathrm{~nm}$ were produced by a fast addition of hydroxylamine hydrochloride. These particles also showed a low size distribution. The occurrence can be seen in Figure 76. From this study, it can be concluded that the fast addition of hydroxylamine hydrochloride produces monodisperse star polymertemplated gold nanoshells. SEM image of the monodisperse particles in higher magnification is shown in Figure 77. The particles were subjected to centrifugation process to remove any excess reagents or by-product salts prior to imaging. 

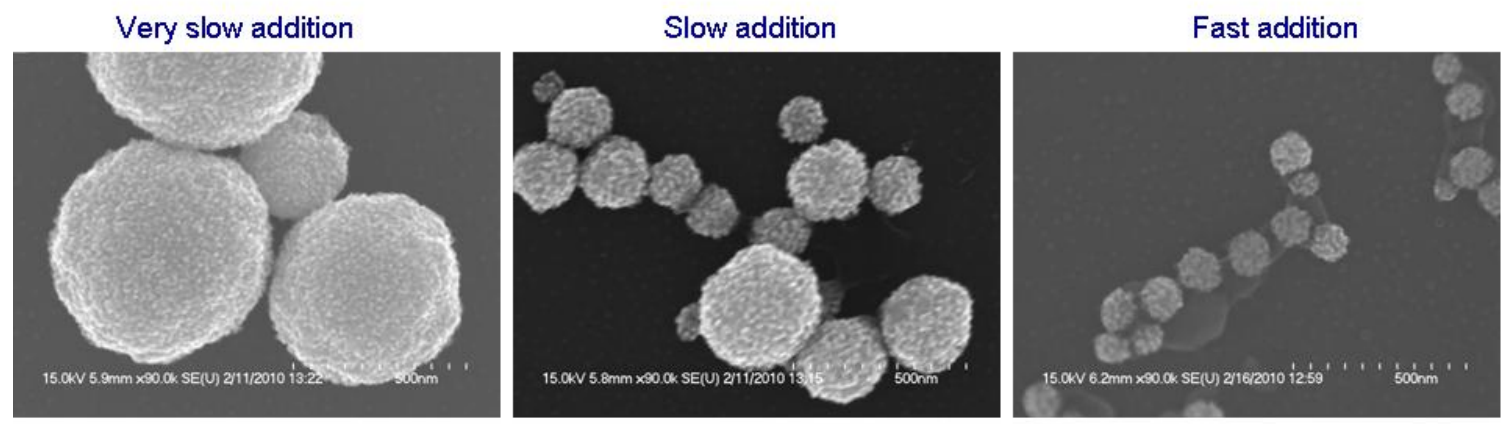

Figure 76. SEM images of star polymer-templated gold nanoshells resulting from three different addition rates of hydroxylamine hydrochloride. The fast addition generated monodisperse particles.

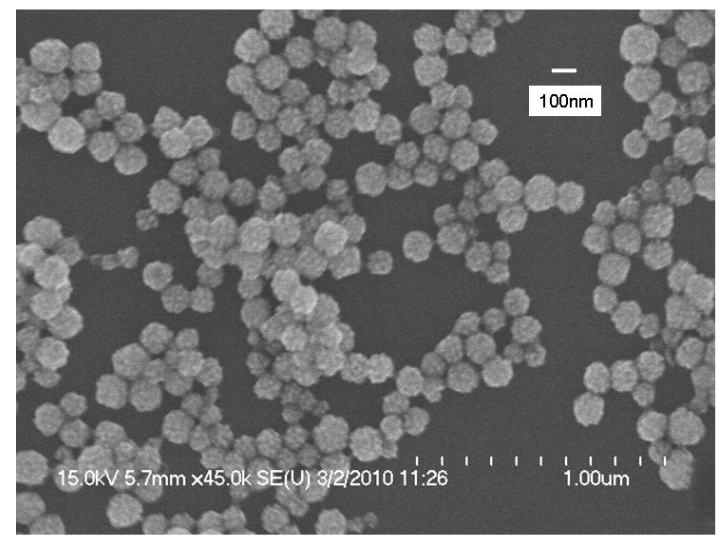

Figure 77. SEM image of $100 \mathrm{~nm}$ gold nanoshells from the fast addition of hydroxylamine hydrochloride in high magnification.

To further verify that star polymers were inside the gold nanoshells, a crosssection SEM was performed. In the sample preparation, the gold nanoparticles were coated with $\mathrm{Al}_{2} \mathrm{O}_{3}$, and the focused ion beam (FIB) technique was used to make the cross-section cut of an individual particle. From the cross-section image, as shown in Figure 78, it seemed there were multiple star polymers instead of a single star polymer inside the gold nanoshells. The thin gold shells displayed the brightest contrast in this image while the dark holes inside the whole particle were presumably the star polymers. 


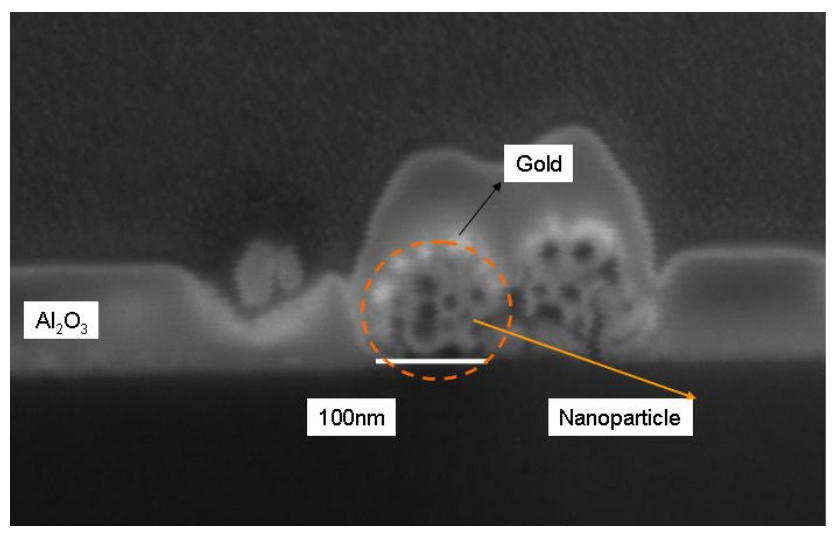

Figure 78. Cross-section SEM image of star polymer-templated gold nanoshells.

In order to further confirm the composition, TEM and EELS analysis were performed. TEM dark-field and bright-field images in Figure 79 clearly showed that the gold particles exhibited a porous structure. The holes/porous were in the range of 15-20 $\mathrm{nm}$ which is the right size to be the diameter of star polymer as measured by light scattering and AFM. Additionally, based on the EELS result, these holes were composed of carbon-rich materials. As shown in Figure 80, alternating traces of gold and carbonrich materials were observed when a scan was performed across one gold particle. This result further verified that star polymers were indeed contained within the gold particle and served as core materials.
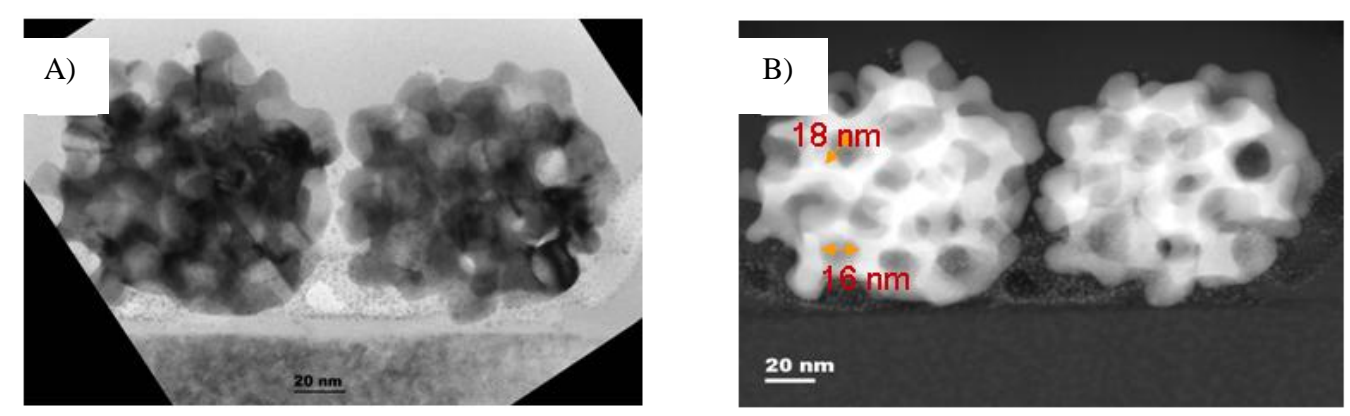

Figure 79. A) bright-field and B) dark-field TEM images of star polymer-templated gold nanoparticles. 


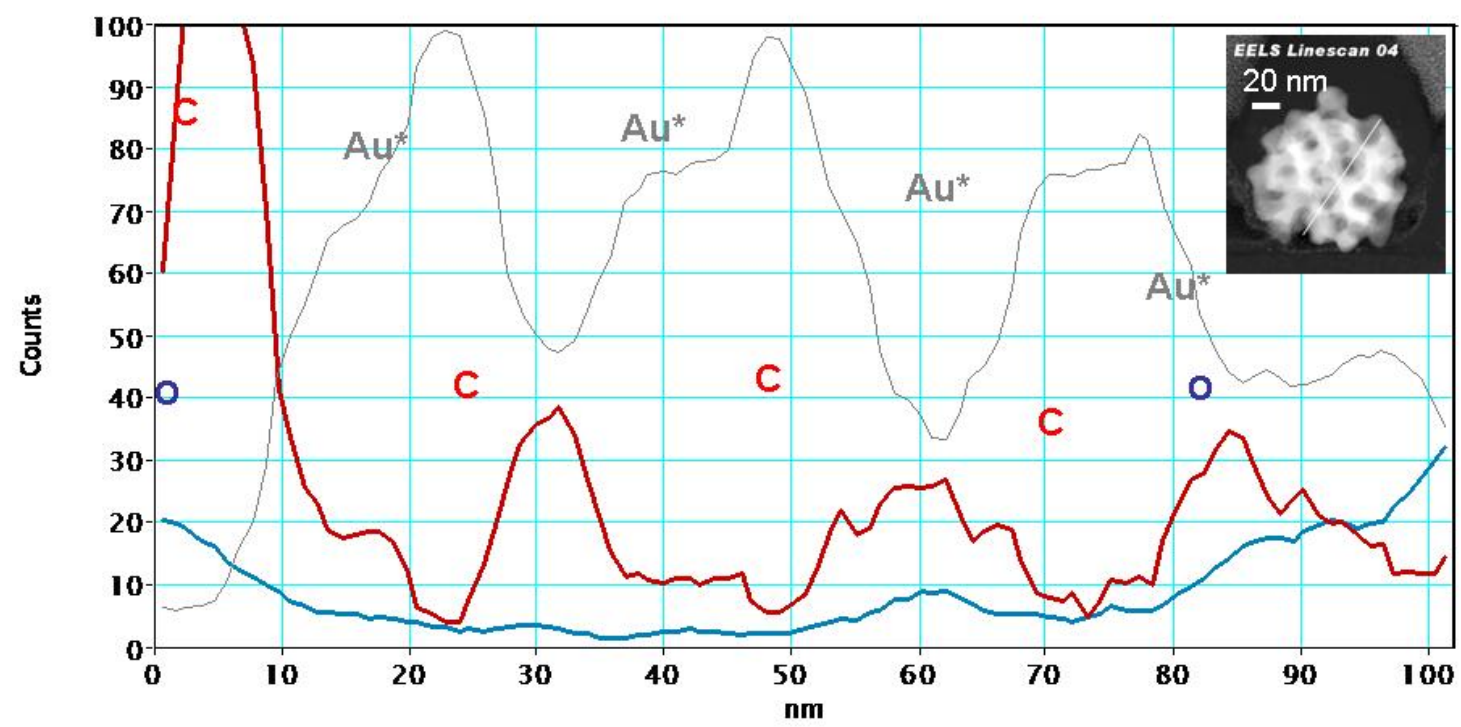

Figure 80. EELS spectra of star polymer-templated gold nanoparticles. A scan was performed across one gold particle as shown by the white line in the inset.

The fact that multiple star polymers were contained in one gold particle is possibly caused by some interactions of the seeds-decorated star polymers with each other during the growth or seeding process. Presuming that the amine groups of the star polymer interact with the negatively charged gold seeds via electrostatic interactions, fully decorated star polymer should repel each other due to the negative charge at the outer seed layer. It is possible, however, that the star polymers are not fully decorated with the seeds, allowing two amine groups from two different star polymers to interact with one seed, as illustrated in Figure 81. 


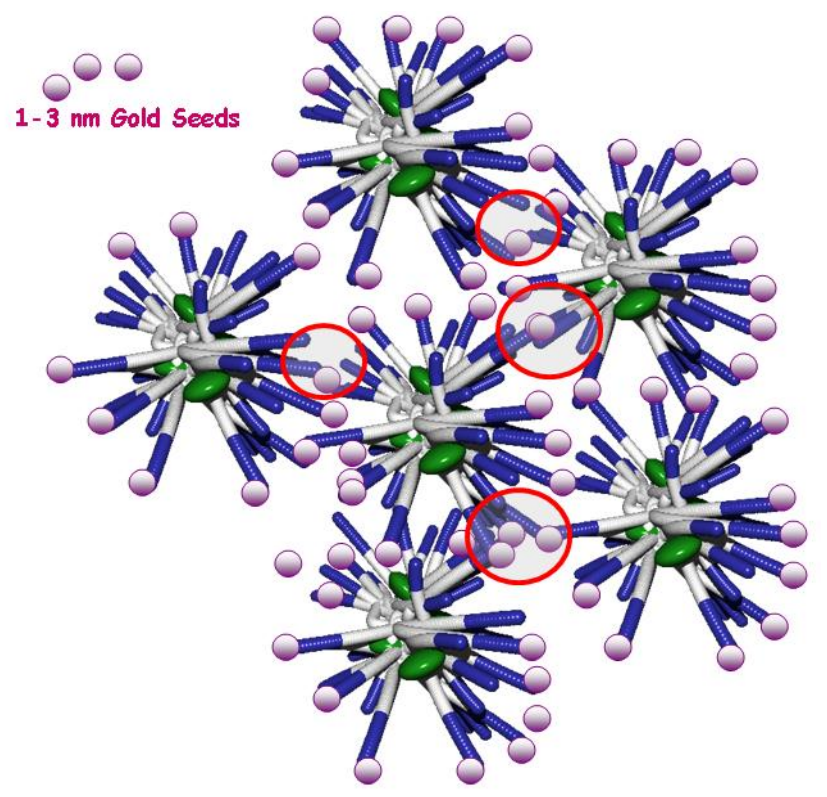

Figure 81. Illustration of possible interparticle interactions between seeds-decorated star polymers (indicated by the red circles).

As mentioned in the materials and methods chapter, free base porphyrins were embedded inside the star polymer via self-assembly process. The porphyrins embedment was aimed to verify the existence of star polymers as core materials and to study the effects of gold nanoshells on the fluorescence of the porphyrins. If star polymer was actually coated with gold shell, and assuming that the porphyrin and gold surface were in the right proximity, the signature peak of porphyrin should theoretically be observable.

When the UV-visible spectrum of gold nanoshells with a mean diameter of 100 $\mathrm{nm}$ was recorded, the porphyrin signature peak at around $420 \mathrm{~nm}$ was observed. An absorption peak at $790 \mathrm{~nm}$ was also observed as a result of the presence of gold nanoshells in the solution. This result supported the above theory. To eliminate the possibility of porphyrin signal due to the uncoated star polymers, a THF dialysis was 
carried out. Due to preferential solubility of porphyrins in THF, porphyrins from the uncoated star polymers should diffuse out of solution and there will be no porphyrin signal. However, if star polymers which contained porphyrins were coated with gold, the gold shell should act as a diffusion barrier and retain the porphyrins inside. UV-visible spectra of the gold nanoshell solution before and after two days of dialysis are shown in Figure 82 . The porphyrin signal after dialysis was still noticeable although the intensity was reduced (at the same intensity of gold shell peak). This finding, however, led to a new scenario where a gold nanoshell provided a porous structure that allowed some of the porphyrins to be leaked out. This may suggest the use of gold nanoshells as a drugdelivery vehicle. Intensive study would be necessary to confirm this potential.

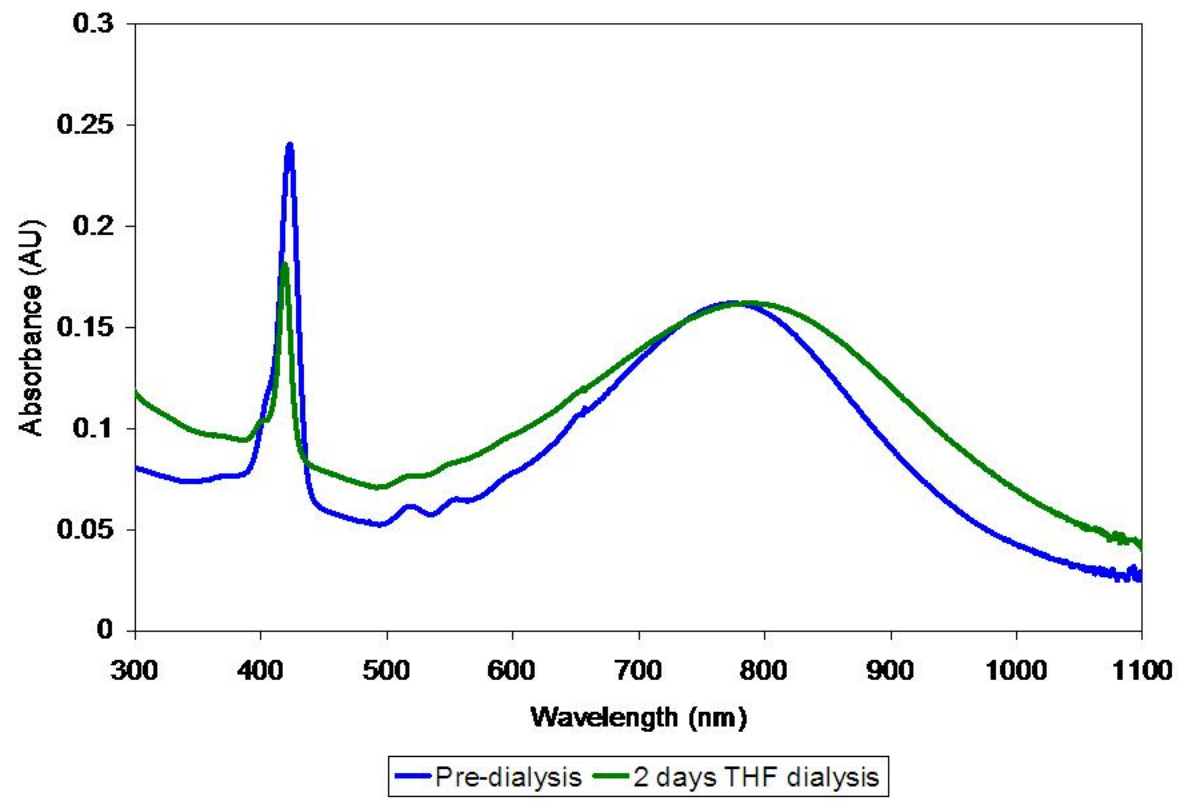

Figure 82. UV-visible spectra of star polymer/porphyrins-templated gold nanoshells before and after two days of THF dialysis. Porphyrin signature peak can be observed at $\sim 420 \mathrm{~nm}$. 
Having demonstrated that monodisperse gold nanoshells with a mean diameter of $100 \mathrm{~nm}$ were feasibly produced and that star polymers were actually contained in the gold particles, the next study was focused on the possibility of enhanced fluorescence by the gold shell. As shown in Figure 83, at the same intensity level of porphyrins at $420 \mathrm{~nm}$ the gold nanoshells displayed an enhancement in the fluorescence emission by two-fold compared to a 'bare' star polymer/porphyrin occlusion complex. This result suggested that the gold nanoshell structure indeed enhanced the fluorescence emission of porphyrins. To further increase the enhancement factor, the proximity of porphyrin molecules and gold shell may be optimized. Modifying the star polymer size or length of the arms can be considered as an option in this case.
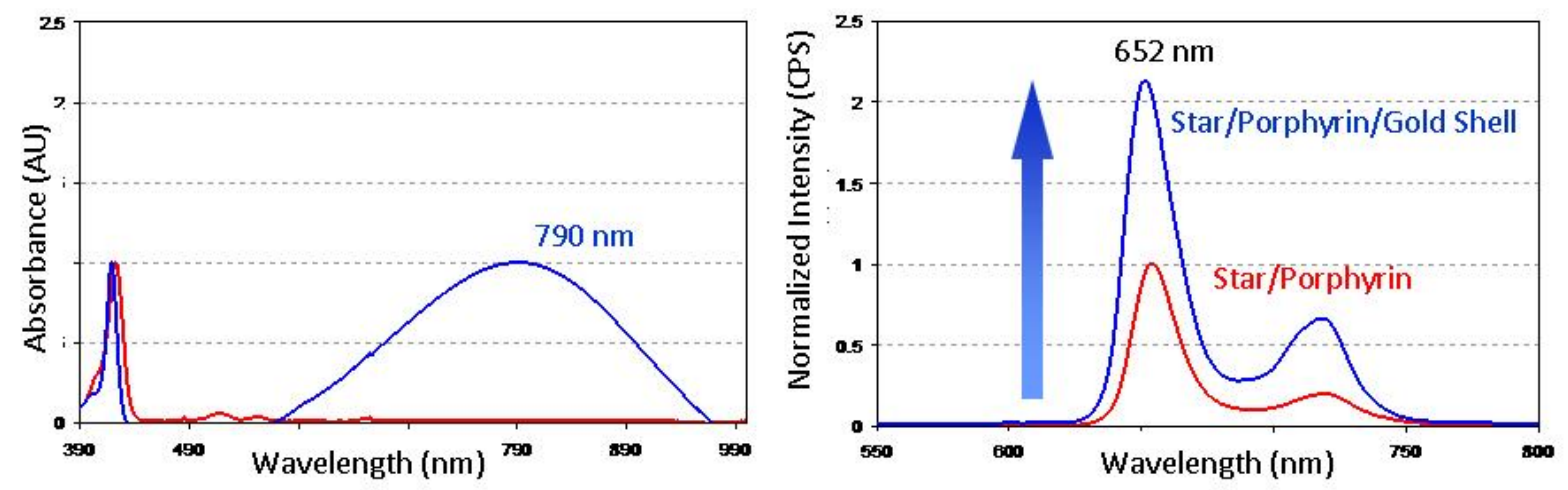

Figure 83. At the same level of porphyrin concentration (left), there was a two-fold enhancement in the emission spectra of the star polymer-templated gold nanoshells compared to that of the 'bare' star polymer/porphyrin (right). 


\section{CHAPTER SIX}

\section{CONCLUSIONS AND FUTURE WORK}

Star polymer PS-DMAEMA was demonstrated to be an excellent template for the formation of 2D and 3D gold nanostructures via electroless deposition. The deposition of star polymers in a surface-confined form (2D) occurred in a sub-second time frame, as verified by SPR spectroscopy. The deposited star polymer film was a monolayer, stable, contiguous, and effective in promoting gold seed attachment. The star polymer also displayed a higher seed attachment rate compared to other templates such as APTMS and PAH. As a result of rapid templating and seeding time, star polymer reduced the total processing time from a typically reported 28 hours to less than one hour.

The growth solution concentration, plating time, and agitation method were shown to be the significant factors in generating gold films with a specific thickness and uniformity. At a constant growth solution concentration, film thickness was shown to be a linear function of plating time. The generated gold films were shown to generate strong SPR signals and display good mechanical stability. The mechanical stability of the star polymer-templated gold films was slightly inferior to that of the evaporated gold but it was comparable to those of the APTMS-templated and PAH-templated gold films. Future work can be carried out to test the chemical stability and reactivity of these electroless gold films by using them as SPR substrates. For instance, deposition of layerby-layer star polymers or attachment of specific bio-conjugation molecules onto the gold films could be investigated. 
Gold nanoshells utilizing star polymers as templates were produced in various sizes and NIR absorptions. The main parameters that determined the particle size were growth solution concentration, seed amount, and the rate of hydroxylamine hydrochloride addition. The fast addition of hydroxylamine hydrochloride generated particles with a low size distribution. Instead of a single star polymer, cross-section SEM and TEM analysis revealed that multiple star polymers were contained in a single gold nanoparticle with a diameter of $100 \mathrm{~nm}$. EELS further verified the existence of star polymers as carbon-rich materials inside the gold nanoparticles. Future work is needed to isolate the seed-decorated star polymers prior to the growth step so that an individual gold nanoshell contains only a single star polymer as the core. Sonication might be a suitable technique to isolate the seed-decorated star polymers. The adjustment of $\mathrm{pH}$ of the seed solution prior to its addition to star polymer solution also merits investigation.

The porphyrin signal after two days of THF dialysis was still noticeable although the intensity was reduced (at the same intensity of gold shell peak). This result indicated that the porphyrins were still contained inside the gold shells. This finding also led to a new scenario where a gold nanoshell provided a porous structure that allowed some of the porphyrins to be leaked out. This may suggest the use of gold nanoshells as a drugdelivery vehicle. Further study is required to confirm this possibility. The presence of gold shell on the star polymer/porphyrin complex also induced a fluorescence enhancement by two-fold compared to that of 'bare' star polymer/porphyrin occlusion complex. To further increase the enhancement factor, the proximity of porphyrin 
molecules and gold shell can be optimized. Modifying the star polymer size or length of the arms can be considered as an option. 


\section{REFERENCES}

1. M.C. Daniel and D. Astruc, "Gold nanoparticles: Assembly, supramolecular chemistry, quantum-size related properties, and applications toward biology, catalysis and nanotechnology," Chem. Rev., 104, 293-346 (2004).

2. A.S.K. Hashmi, "Gold-catalyzed organic reactions," Chem. Rev., 107, 3180-3211 (2007).

3. G. Tsutsui, S. Huang, H. Sakaue, S. Shingubara and T. Takahagi, "Well-size controlled colloidal gold nanoparticles dispersed in organic solvents, " Jpn. J. Appl. Phys., 40, 346-349 (2001).

4. A.K. A. Swami, M. D'Costa, R. Pasricha and M. Sastry, "Variation in morphology of gold nanoparticles synthesized by the spontaneous reduction of aqueous chloroaurate ions by alkylated tyrosine at a liquid-liquid and air-water interface," J. Mater. Chem., 14, 2696-2702 (2004).

5. L.G.a.C.J.M. N.R. Jana, "Seeding growth for size control of 5-40 $\mathrm{nm}$ diameter gold nanoparticles, " Langmuir, 17, 6782-6786 (2001).

6. H.J. Chen, Y.L. Wang, Y.Z. Wang, S.J. Dong and E.K. Wang, "One-step preparation and characterization of PDDA-protected gold nanoparticles," Polymer, 47, 763-766 (2006).

7. K. Torigoe and K. Esumi, "Preparation of colloidal gold by photoreduction of tetracyanoaurate(1-)-cationic surfactant complexes, " Langmuir, 8 (1), 59-63 (1992).

8. Y. Bai, S. Zhao, K. Zhang and C.Q. Sun, "Covalently attached multilayer assemblies of citrate-capped colloidal gold nanoparticles and diazo-resins," Colloids Surf., A, 281, 105-112 (2006).

9. E. Papazoglou and A. Parthasarathy, Bionanotechnology, (Morgan and Claypool, 2007), pp. 24. 2007.

10. H. Miyake, S. Ye and M. Osawa, "Electroless deposition of gold thin films on silicon for surface-enhanced infrared spectroelectrochemistry, "Electrochem. Commun., 4, 973-977 (2002).

11. D. Enders, S. Rupp, A. Küller and A. Pucci, "Surface enhanced infrared absorption on Au nanoparticle films deposited on $\mathrm{SiO}_{2} / \mathrm{Si}$ for optical biosensing: Detection of the antibody-antigen reaction," Surf. Sci., 600, L305-L308 (2006). 
12. H.F. Wang, Y.G. Yan, S.J. Huo, W.B. Cai, Q.J. Xu and M. Osawa, "Seeded growth fabrication of Cu-on-Si electrodes for in situ ATR-SEIRAS applications," Electrochim. Acta, 52, 5950-5957 (2007).

13. F. Guan, M. Chen, W. Yang, J.Q. Wang, S.R. Yong and Q.J. Xue, "Fabrication of patterned gold microstructure by selective electroless plating," Appl. Surf. Sci., 240, 24-27 (2005).

14. S. Schaefers, L. Rast and A. Stanishevsky, "Electroless silver plating on spincoated silver nanoparticle seed layers,," Mater. Lett., 60, 706-709 (2006).

15. A. Hilmi and J.H.T. Luong, "Electrochemical detectors prepared by electroless deposition for microfabricated electrophoresis chips, " Anal. Chem., 72 (19), 4677-4682 (2000).

16. W. Shi, Y. Sahoo, M.T. Swihart and P.N. Prasad, "Gold nanoshells on polystyrene cores for control of surface plasmon resonance, "Langmuir, 21, 16101617 (2005).

17. A.M. Schwartzberg, T.Y. Olson, C.E. Talley and J.Z. Zhang, "Synthesis, characterization, and tunable optical properties of hollow gold nanospheres, " J. Phys. Chem. B, 110, 19935-19944 (2006).

18. C. Graf and A.V. Blaaderen, "Metallodielectric colloidal core-shell particles for photonic applications," Langmuir, 18, 524-534 (2002).

19. X. Ji, R. Shao, A.M. Elliot, R.J. Stafford, E. Esparza-Coss, J.A. Bankson, G. Liang, Z.P. Luo, K. Park, J.T. Markert and C. Li, "Bifunctional gold nanoshells with a superparamagnetic iron oxide-silica core suitable for both MR imaging and photothermal therapy," J. Phys. Chem. C, 111, 6245-6251 (2007).

20. J. Lee, J. Yang, H. Ko, S.J. Oh, J. Kang, J. Son, K. Lee, S. Lee, H. Yoon, J. Suh, Y. Huh and S. Haam, "Multifunctional magnetic gold nanocomposites: Human epithelial cancer detection via magnetic resonance imaging and localized synchronous therapy," Adv. Funct. Mater., 18, 258-264 (2008).

21. D. Baudrand and J. Bengston, "Electroless plating processes: Developing technologies for electroless nickel, palladium, and gold, "Met. Finish., 93 (9), 5557 (1995).

22. G.O. Mallory and J.B. Hajdu, Electroless plating: Fundamentals and applications, (William Andres, 1990), pp. 1-2. 1990. 
23. P.C. Hidberg, P.F. Nealey, W. Helbig and G.M. Whitesides, "New strategy for controlling the size and shape of metallic features formed by electroless deposition of copper: Microcontact printing of catalyst on oriented polymers, followed by thermal shrinkage," Langmuir, 12 (21), 5209-5215 (1996).

24. Y. Lei, H.Y. Chen, H.P. Dai, Z.R. Zeng, Y. Lin, F.M. Zhou and D.W. Pang, "Electroless-plated gold films for sensitive surface plasmon resonance detection of white spot syndrome virus, " Biosens. Bioelectron., 23, 1200-1207 (2008).

25. S. Hrapovic, Y.L. Liu, G. Enright, F. Bensebaa and J.H.T. Luong, "New strategy for preparing thin gold films on modified glass surfaces by electoless deposition," Langmuir, 19, 3958-3965 (2003).

26. H.W. Huang, S.F. Zhang, L. Qi, X. Yu and Y. Chen, "Microwave-assisted deposition of uniform thin gold film on glass surface," Surf. Coat. Technol., 200, 4389-4396 (2006).

27. M. Brust and C.J. Kiely, "Some recent advances in nanostructure preparation from gold and silver particles: a short topical review," Colloids Surf., A, 202, 175-186 (2002).

28. C.A. Dai, Y.L. Wu, Y.H. Lee, C.J. Chang and W.F. Su, "Fabrication of 2D ordered structure of self-assembled block copolymers containing gold nanoparticles," J. Cryst. Growth, 288, 128-136 (2006).

29. T.N. Vorobyova, S.K. Poznyak, A.A. Rimskaya and O.N. Vrublevskaya, "Electroless gold plating from hypophosphite-dicyanoaurate bath," Surf. Coat. Technol., 176, 327-336 (2004).

30. C.D. Iacovangelo, "Autocatalytic electroless gold deposition using hydrazine and dimehtylamine borane as reducing agents, " J. Electrochem. Soc., 138, 976-982 (1991).

31. H. Menzel, M.D. Mowery, M. Cai and C.E. Evans, "Surface-confined nanoparticles as substrate for photopolymerizable self-assembled monolayers," Adv. Mater., 11 (2), 131-134 (1999).

32. S. Meltzer, R. Resch, B.E. Koel, M.E. Thompson, A. Madhukar, A.A.G. Requicha and P. Will, "Fabrication of nanostructure by hydroxylamine seeding of gold nanoparticle templates," Langmuir, 17 (5), 1713-1718 (2001).

33. K.R. Brown and M.J. Natan, "Hydroxylamine seeding of colloidal Au nanoparticles in solution and on surfaces, "Langmuir, 14 (4), 726-728 (1998). 
34. M.D. Musick, D.J. Pea, S.L. Botsko, T.M. McEvoy, J.N. Richardson and M.J. Natan, "Electrochemical properties of colloidal Au-based surfaces: Multilayer assemblies and seeded colloid films," 15 (3), 844-850 (1999).

35. UK Surface Analysis Forum. SPR - Surface plasmon resonance [Online]. Available at <http://www.uksaf.org/tech/spr.html> (accessed 30 January 2009).

36. Biacore. Using surface plasmon resonance (SPR) [Online]. Available at $\leq \mathrm{http}: / / \mathrm{www}$. biacore.com/lifesciences/technology/introduction/following_interact ion/Detecting_interactions_in_real_time/index.html?backurl=\%2Flifesciences $\% 2$ Ftechnology\%2Fintroduction\%2Ffollowing interaction\%2Findex.html> (accessed 30 January 2009).

37. R.B.M. Schasfoort and A.J.Tudos, Handbook of surface plasmon resonance, (Springer-Verlag New York, LLC, 2008), pp. 1-5. 2008.

38. D.R. Shankaran, K.V. Gobi and N. Miura, "Recent advancement in surface plasmon resonance immunosensors for detection of small molecules of biomedical, food and environmental interest, " Sens. Actuators, B, 121, 158-177 (2007).

39. J. Homola, S.S. Yee and G. Gauglitz, "Surface plasmon resonance sensors: review," Sens. Actuators, B, 54, 3-15 (1999).

40. J. Homola, "Present and future of surface plasmon resonance biosensors, " Anal. Bioanal. Chem., 377, 528-539 (2003).

41. Y.D. Jin, X.F. Kang, Y.H. Song, B.L. Zhang, G.J. Cheng and S.J. Dong, "Controlled nucleation and growth of surface-confined gold nanoparticles on a (3-aminopopyl)trimethoxysilane-modified glass slide: A strategy for SPR substrates," Anal. Chem., 73 (13), 2843-2849 (2001).

42. R.M. Crooks, B.I. Lemon, L. Sun, L.K. Yeung and M.Q. Zhao, "Dendrimerencapsulated metals and semiconductors: Synthesis, characterization, and applications," Top. Curr. Chem., 212, 81-135 (2001).

43. J. Sly, S.M. Elliot, V.Y. Lee and R.D. Miller, "Versatile polyvalent support architectures for nano-scale constructs," Unpublished results (2007).

44. J. Sly, IBM Almaden Research Center, private communication (February 2008).

45. C. Loo, A. Lin, L. Hirsch, M. Lee, J. Barton, N. Halas, J. West and R. Drezek, "Nanoshell-enabled photonic-based imaging and therapy of cancer," Techol. Cancer Res. T., 3 (1), 33-40 (2004). 
46. S.J. Oldenburg, R.D. Averitt, S.L. Westcott and N.J. Halas, "Nanoengineering of optical resonances," Chem. Phys. Lett., 288, 243-247 (1998).

47. J.S. Shumaker-Parry, R. Aebersold and C.T. Campbell, "Parallel, quantitative measurement of protein binding to a 120-element double-stranded DNA array in real time using surface plasmon resonance microscopy," Anal. Chem., 76, 20712082 (2004).

48. X. Liu, D.Q. Song, Q.L. Zhang, Y. Tian, Z.Y. Liu and H.Q. Zhang, "Characterization of drug-binding levels to serum albumin using a wavelength modulation surface plasmon resonance sensor," Sens. Actuators, B, 117, 188-195 (2006).

49. X.D. Su, C.Y. Lin, S.J. O'Shea, H.F. The, W.Y.X. Peh and J.S. Thomsen, "Combinational application of surface plasmon resonance spectroscopy and quartz crystal microbalance for studying nuclear hormone receptor-response element interactions," Anal. Chem., 78, 5552-5558 (2006).

50. B.K. Oh, Y.K. Kim, K.W. Park, W.H. Lee and J.W. Choi, "Surface plasmon resonance immunosensor for the detection of Salmonella typhimurium," Biosens. Bioelectron., 19, 1497-1504 (2004).

51. N. Khlebtsov, G. Akchurin, B. Khlebtsov, G. Akchurin, V. Tuchin and V. Zharov, "Laser-induced destruction of gold nanoshells: new weapons in the cell-killing arsenal," SPIE Newsroom (2008).

52. K.C. Grabar, K.J. Allison, B.E. Baker, R.M. Bright, K.R. Brown, R.G. Freeman, A.P. Fox, C.D. Keating, M.D. Musick and M.J. Nathan, "Two-dimensional arrays of colloidal gold particles: A flexible approach to macroscopic metal surfaces," Langmuir, 12 (10), 2353-2361 (1996).

53. S. Watanabe and S.L. Regen, "Dendrimers as building blocks for multilayer construction," J. Am. Chem. Soc., 116 (19), 8855-8856 (1994).

54. M. Wells and R.M. Crooks, "Interactions between organized, surface-confined monolayers and vapor-phase probe molecules. 10. Preparation and properties of chemically sensitive dendrimer surfaces, "J. Am. Chem. Soc., 118 (16), 3988-398 (1996).

55. H. Tokuhisa and R.M. Crooks, "Interactions between organized, surface-confined monolayers and vapor phase probe molecules. 12. Two new methods for surfaceimmobilization and functionalization of chemically sensitive dendrimer surfaces," Langmuir, 13 (21), 5608-5612 (1997). 
56. V.V. Tsukruk, F. Rinderspacher and V.N. Bliznyuk, "Self-assembled multilayer films from dendrimers, " Langmuir, 13 (8), 2171-2176 (1997).

57. C.S. Bonifacio, "Self-assembled layer-by-layer star polymers by electrostatic interactions," Graduate Thesis, San Jose State University (2008).

58. L. Chang, "Effect of coordination chemistry on layer-by-layer deposition of star polymers," Graduate Thesis, San Jose State University (2008).

59. K.C. Grabar, R.G. Freeman, M.B. Hommer and M.J. Nathan, "Preparation and characterization of Au colloid monolayers, " Anal. Chem., 57, 735-743 (1995).

60. J.W. Zheng, Z.H. Zhu, H.F. Chen and Z.F. Liu, "Nanopatterned assembling of colloidal gold nanoparticles on silicon," Langmuir, 16 (10), 4409-4412 (2000).

61. L.W. Shive and I.J. Malik. Method of etching SiO2 and process of cleaning silicon wafers using dilute chemical etchants and a megasonic field [Online]. Available at 〈http://www.freepatentsonline.com/EP0844650.html> (accessed 30 January 2009).

62. S.T. Liu, T. Zhu, R.S. Hu and Z.F. Liu, "Evaporation-induced self-assembly of gold nanoparticles into a highly organized two-dimensional array," Phys. Chem. Chem. Phys., 4, 6059-6062 (2002).

63. S.K. Kang, Y.H. Kim, I.H. Choi, J.W. Lee and J.H. Yi, "An array of Au nanoparticles on the nanopatterned Si(100), " Microelectron. Eng., 81, 389-393 (2005).

64. C.X. Guo, P. Boullanger, L. Jiang and T. Liu, "Highly sensitive gold nanoparticles biosensor chips modified with a self-assembled bilayer for detection of Con A," Biosens. Bioelectron., 22, 1830-1834 (2007).

65. H.Y. Jung, Y.K. Park, S.H. Park and S.K. Kim, "Surface enhanced Raman scattering from layered assemblies of close-packed gold nanoparticles, " Anal. Chim. Acta., 602, 236-243 (2007).

66. D.I. Gittins, A.S. Susha, B. Schoeler and F. Caruso, "Dense nanoparticulate thin films via gold nanoparticle self-assembly," Adv. Mater., 14 (7), 508-511 (2002).

67. S.L. Westcott, S.J. Oldenburg, T.R. Lee and N.J. Halas, "Formation and adsoprtion of clusters of gold nanoparticles onto functionalized silica nanoparticle surfaces," Langmuir, 14, 5396-5401 (1998). 
68. T. Pham, J.B. Jackson, N.J. Halas and T.R. Lee, "Preparation and characterization of gold nanoshells coated with self-assembled monolayers," Langmuir, 18, 4915-4920 (2002).

69. S. Park, M. Park, P. Han and S. Lee, "The effect of pH-adjusted gold colloids on the formation of gold clusters over APTMS-coated silica cores, "Bull. Korean Chem. Soc., 27 (9), 1341-1345 (2006).

70. Y.T. Lim, O.O. Park and H. Jung, "Gold nanolayer-encapsulated silica particles synthesized by surface seeding and shell growing method: near infrared responsive materials, "J. Colloid Interf. Sci, 263, 449-453 (2003).

71. L.A. Baker, F.P. Zamborini, L. Sun and R.M. Crooks, "Dendrimer-mediated adhesion between vapor-deposited Au and glass or Si wafer," Anal. Chem., 71 (19), 4430-4406 (1999).

72. D.G. Duff and A. Baiker, "A new hydrosol of gold clusters. 2. A comparison of some different measurement techniques," Langmuir, 9, 2310-2317 (1993).

73. H.S. Fogler, Elements of chemical reaction engineering, (Prentice-Hall, Inc., 2006), pp. 771-780. 2006.

74. Y. Okinaka, R. Sard, C. Wolowodiuk, W.H. Craft and T.F. Retajczyk, "Some practical aspects of electroless gold plating," J. Electrochem. Soc., 121 (1), 56-62 (1974).

75. S. Eustis and M.A. El-Sayed, "Why gold nanoparticles are more precious than pretty gold: Noble metal surface plasmon resonance and its enhancement of the radiative and nonradiative properties of nanocrystals of different shapes, "Chem. Soc. Rev., 35, 209-207 (2006).

76. R. Bardhan, N.K. Grady, J.R. Cole, A. Joshi and N.J. Halas, "Fluorescence enhancement by Au nanostructures: Nanoshells and nanorods," ACS Nano, 3 (3), 744-752 (2009).

77. M. Park, S. Lim, S. Lee and S. Park, "Relative parameter contributions for encapsulating silica-gold nanoshells by Poly(N-isopropylacrylamide-co-acrylic acid) hydrogels," Macromol. Res., 17 (5), 307-312 (2009).

78. D. Pissuwan, S.M. Valenzuela and M.B. Cortie, "Therapeutic possibilities of plasmonically heated gold nanoparticles," Trends Biotechol., 24 (2), 62-67 (2006).

79. B. Haley and E. Frenkel, "Nanoparticles for drug delivery in cancer treatment," Urol. Oncol_Semin. O. I. , 26, 57-64 (2008). 
APPENDIX: An Example of Standard Deviation Calculation.

The following calculations show how a standard deviation is determined.

Film thickness: $62.14,65.18,60.42 \mathrm{~nm}$

Mean value: $(62.14+65.18+60.42) / 3 \mathrm{~nm}=62.58 \mathrm{~nm}$

List of deviations: $-0.44,2.6,-2.16$

Squares of deviation: $0.1936,6.76,4.6656$

Sum of deviations: 11.6192

Standard deviation $=(11.6192 / 2)^{\wedge} 0.5=2.41$ 